\title{
Fracture and Fatigue Analysis of Welded Structures Using Finite Element Analysis
}

\author{
by \\ Denys Vodzyk \\ A thesis submitted to \\ the Faculty of Graduate and Postdoctoral Affairs \\ in partial fulfilment of \\ the requirements for the degree of \\ Master of Applied Science \\ in \\ Mechanical Engineering \\ Department of Mechanical and Aerospace Engineering \\ Carleton University \\ Ottawa, Ontario, Canada \\ December 2016
}

Copyright (C)

2016 - Denys Vodzyk 
The undersigned recommend to

the Faculty of Graduate and Postdoctural Affairs

acceptance of the thesis

\title{
Fracture and Fatigue Analysis of Welded Structures Using Finite Element Analysis
}

\author{
Submitted by Denys Vodzyk \\ in partial fulfilment of the requirements for the degree of \\ Master of Applied Science
}

Dr. John A. Goldak, Thesis Supervisor

Dr. Ronald Miller, Department Chair

Carleton University

2016 


\section{Abstract}

Welded joints that are subjected to cyclic operational conditions tend to fail due to fatigue failure. This type of failure can occur at a stress level below the yield strength of the material. Design to resist such failures and the early detection of the internal flaws are the basic components of the damage tolerance design philosophy, which have significant impact in terms of saving time, money and people's lives.

Computational analysis of welded structures has become a major tool in performing fatigue analysis of welded structures and conducting conventional experimental tests. With the help of an accurate FEA analysis one can estimate the fatigue life of a structure and improve it by optimization.

Current thesis work focuses on an automated fracture and fatigue analysis of welded components. Fracture and fatigue analysis in this research starts with a computation of the transient temperature, microstructure, stress and displacement in welds. The set of the Gauss points that have the largest tensile principal stresses are determined for the purpose of locating candidate sites of cracks. The crack mesh, for computing the stress intensity factor associated with the crack tip for a fracture mechanics analysis, is created. Material force, J-integral, stress intensity factor and CTOA are computed from the results of the stress analysis. Result of the SIF is compared with the fracture toughness. The increment in the stress intensity factor, associated with the crack tip for a fatigue load cycle, is computed. The rate of the crack growth per load cycle in this work is calculated by solving the Paris-Erdogan equation, based on the increment 
in the stress intensity factor.

Verification of the VrWeld software is done by comparing the stress results with an analytical solution. Recommendations are made based on the obtained results and the directions of future work are suggested. 
I would like to dedicate this thesis to my parents, Inna and Petro Vodzyk, for their love, encouragement and support. 


\section{Acknowledgments}

I would like to express my sincere appreciation to my supervisor, Professor John A. Goldak, for his continuous support and guidance throughout the journey of this work. This thesis would not be accomplished without his constant involvement and contribution into my research. No words exist to express the role he played in my life.

I wish to express my gratitude to Goldak Technologies Inc, especially to Mr. Stanislav Tchernov and Mr. Jianguo Zhou for their patience while working on this project and support with the software. I would like to thank my colleagues Hossein Nimrouzi and Komeil Kazemi for their guidance and support.

Finally, I want to thank my wife, Valentyna Artemchuk for all the support and encouragement she gave me during the period of this thesis. 


\section{Contents}

Abstract $\quad$ iii

Acknowledgments $\quad$ vi

Table of Contents vii

List of Figures $\quad \mathrm{x}$

List of Acronyms $\quad$ xi

1 Introduction 1

1.1 Background ......................... 1

1.2 Research Strategy . . . . . . . . . . . . . . . . . . 3

1.3 Directional scope of this study . . . . . . . . . . . . . 4

1.4 Objectives . . . . . . . . . . . . . . . . . . . . . . 7

1.5 Scope and Organization of the Thesis . . . . . . . . . . . . . . . . . . 7

2 Fracture mechanics theory $\quad 9$

2.1 Fracture mechanics concept . . . . . . . . . . . . . . . . . 9

2.2 Linear elastic fracture mechanics . . . . . . . . . . . . . . . . . 10

2.3 Crack initiation . . . . . . . . . . . . . . . . . 12

2.4 Crack tip plastic zone . . . . . . . . . . . . . . . . . . . . . 12

2.5 Stress intensity factor . . . . . . . . . . . . . . . . 13 
2.6 Crack propagation and Paris-Erdogan equation . . . . . . . . . 16

2.7 Energy release rate . . . . . . . . . . . . . . . . . . . . 17

2.8 Material force method . . . . . . . . . . . . . . . . . 20

2.9 Calculation of the SIF from the Crack Tip Opening Angle near the crack tip . . . . . . . . . . . . . . . . . . . 22

3 Fatigue analysis assessment methods $\quad 24$

3.1 Introduction . . . . . . . . . . . . . . . . 24

3.2 Properties of S-N curve . . . . . . . . . . . . . . . 26

3.2.1 Stress life . . . . . . . . . . . . . 26

3.2.2 Strain life . . . . . . . . . . . . . . . . . . . . . . . 27

4 Finite element analysis $\quad 28$

4.1 Problem analysis . . . . . . . . . . . . . . . . . . . . 28

4.1.1 Two-dimensional stress analysis of a plate with a hole . . . . . 28

4.1.1.1 Determination of the maximum stress . . . . . . . 28

4.1.1.2 Determination of the material force vectors . . . . 31

4.1.2 Stress analysis of an edge crack in a finite width sheet . . . . . 34

4.1.2.1 Procedure of a two dimensional manual crack analysis 34

4.1.2.2 Determination of the stress intensity factor . . . . 36

4.1.2.3 Comparison of the stress at the crack tip . . . . . . 40

4.1.2.4 Determination of the number of cycles for a fatigue crack growth . . . . . . . . . . . . . . . . 42

4.2 Manual fatigue analysis of the laser weld . . . . . . . . . . . . . 43

4.2.1 Problem geometry ................... 43

4.2.2 Setup and analysis . . . . . . . . . . . . . . . . . 44

4.3 Automation of the fatigue analysis process . . . . . . . . . . . . 48

4.3.1 Description of the wizard for an automated analysis . . . . . 50 
4.4 Automated analysis of the CTOA of the crack faces . . . . . . . 55

4.5 Automated fatigue analysis of the WIC test and girth weld . . . . . . 60

4.5.1 Introduction and geometry of the specimens . . . . . . . . . 60

4.5.2 Setup and analysis of the test cases . . . . . . . . . . . . 62

5 Conclusion and possible future works $\quad 65$

5.1 Future direction of work . . . . . . . . . . . . . 67

$\begin{array}{lr}\text { References } & 68\end{array}$

Appendix A Auto-report of the fracture analysis of the WIC test 72

Appendix B Auto-report of the fatigue analysis of the pipe girth weld $\begin{array}{lr}\text { for the crack length of } 4 \mathrm{~mm} & 80\end{array}$

Appendix C Auto-report of the fatigue analysis of the pipe girth weld $\begin{array}{ll}\text { for the crack length of } 6 \mathrm{~mm} & 88\end{array}$ 


\section{List of Figures}

2.1 The three fracture modes $[11] \ldots \ldots \ldots$. . . . . . . . . . 11

2.2 Plastic zone size $[12] \ldots \ldots \ldots \ldots$. . . . . . . . . . . . . . . 11

2.3 Plots of ratios of asymptotic forms to full stresses, as functions of $\mathrm{r} / \mathrm{a}$, adjacent to the tip of a buried crack in an elastic body, subjected to tensile stress. Where $\sigma_{22}$ at $\theta=0$ corresponds to the tensile stress normal to the crack [16]. . . . . . . . . . . . . . . . . . 13

2.4 Polar and Cartesian coordinate systems at the crack tip that are used in LEFM [10]. . . . . . . . . . . . . . . . . . . . . . . . . . . . . 14

2.5 $\sigma_{y y}$ stress on the crack plane for a mode l loading of the crack [10] . . 14

2.6 Three stages of fatigue crack growth [27] . . . . . . . . . . . . . 18

2.7 Evaluation of the SIF from the the crack tip opening angle (CTOA) [38]. 23

3.1 Stress variation in a fatigue load cycle [41] . . . . . . . . . . . 25

3.2 Typical S-N Curve [43]. . . . . . . . . . . . . . . . . . . 26

4.1 Two-dimensional model of the plate with following parameters: $W=$

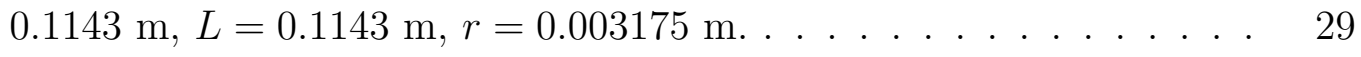

4.2 Mesh used for FEA analysis. . . . . . . . . . . . . . . . . . . . 30

4.3 The result of the FEA stress analysis on a plate with a hole. . . . . . 31

4.4 a) Two-dimensional model of a plate with a hole and b) the result of the material force vectors taken from the literature [46] . . . . . . . . 32

4.5 Two-dimensional plate with a hole for FEA analysis . . . . . . . . . 33 
4.6 Material force vectors magnitude computed with VrSuite. . . . . . . . 33

4.7 Displacement field in the 3D plate without crack with prescribed displacement BCs at the top and bottom ends of $0.00001 \mathrm{~m}$. . . . . . . 37

4.8 a) Displacement in the $2 \mathrm{D}$ plate with a crack and b) displacement field magnified 1000 times in the $2 \mathrm{D}$ plate with a crack. . . . . . . . . 38

4.9 Two dimensional crack plane. . . . . . . . . . . . . . . . . . 39

4.10 a) Material force vectors are shown near the crack tip and b) zoomed in section showing the material force vectors near the crack tip. The vector shown in red is the material force vector acting on the crack tip. 40

4.11 a) The computed nodal stress in the $\mathrm{xx}$ direction for the $2 \mathrm{D}$ plate with the crack and b) zoomed in result of the nodal stress in the xx direction for the $2 \mathrm{D}$ plate with the crack. . . . . . . . . . . .

4.12 Comparison of the stresses at the crack tip computed by FEA with analytical solution derived by LEFM. . . . . . . . . . . . . .

4.13 Geometry of the specimen. Triad axes: red is the $\mathrm{X}$ axis, yellow is the $\mathrm{Y}$ axis, green is the $\mathrm{Z}$ axis. . . . . . . . . . . . . . .

4.14 a) Result of the Gauss points that show a principal stress in the structure b) Zoomed in region of the Gauss points with narrow range of the maximum values. . . . . . . . . . . . . . . .

4.15 Example of an elliptical crack mesh that is perpendicular to the $2 \mathrm{D}$ plane strain mesh . . . . . . . . . . . . . . . . . . . . . . 46

4.16 Material force vectors competed with VrSuite for a crack length of 0.5

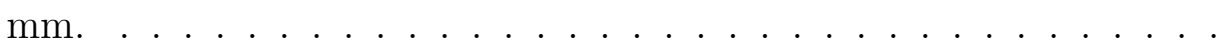

4.17 Material force vectors competed with VrSuite for a crack length of 0.7

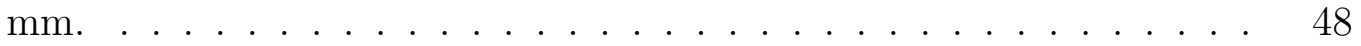

4.18 First step of an automated crack wizard . . . . . . . . . . . 52

4.19 Second step of an automated crack wizard . . . . . . . . . . 53 
4.20 Third step of an automated crack wizard . . . . . . . . . . . . 54

4.21 Fourth step of an automated crack wizard . . . . . . . . . . . . 55

4.22 Displacement at the crack tip of an analysis with an elastic material sharp crack and an analysis with an elastic-plastic material - blunting crack. . . . . . . . . . . . . . . . 56

4.23 Geometry of the 3D object. . . . . . . . . . . . . 57

4.24 a) Traction $\mathrm{BC}$ applied to the 3D object vs time step b) Displacement vs time step for the 3D object. . . . . . . . . . . . . . . 57

4.25 Displacement vs Distance from the crack tip for the time step five in Figure 4.24. See Figure 4.22 for a schematic of elastic and the elastic-plastic displacement for given applied traction BC. . . . . . . . 58

4.26 The result of the crack tip blunting evolution is plotted vs the applied stress for the load steps shown in Figure 4.24. . . . . . . . . . . . . 60

4.27 a) Geometry of the WIC specimen with turned on mesh b) Cross-section of the weld joint of the WIC specimen. . . . . . . . . . . . . . . . 61

4.28 a) a) Geometry of the pipe girth weld b) cross-section of the weld joint of the pipe girth weld. . . . . . . . . . . . . . . . .

4.29 a) Location of the crack in the WIC test specimen b) An elliptical crack mesh with perpendicular to it $2 \mathrm{D}$ crack plane in the WIC test specimen with turned-off parts. . . . . . . . . . . . . . . .

4.30 a) Location of the crack in the pipe weld b) an elliptical crack mesh shown in light green with the plane strain $2 \mathrm{D}$ mesh perpendicular to the crack tip in the pipe girth weld. The pipe parts are made invisible to show the crack and the plane strain 2D mesh. . . . . . . . . . . . . 64

A.1 The displacement field in a 2D plane strain mesh with a crack is shown. 74

A.2 The displacement field in a 2D plane strain mesh with a crack is shown. Magnified 5 times. . . . . . . . . . . . . . . . 
A.3 The xx stress is shown in a 2D plane strain mesh with a crack. . . . . 76

A.4 The computed material force vectors near the crack tip in a $2 \mathrm{D}$ plane strain mesh. . . . . . . . . . . . . . . . . . . . . . . 77

A.5 Plot of the stress vs distance from the crack tip down in the plane of the crack. . . . . . . . . . . . . . . . . . . . . 78

A.6 Plot of the displacement vs distance from the crack tip to the distance of one element in the crack opening direction. . . . . . . . . . . . . 79

B.1 The displacement field in a 2D plane strain mesh with a crack is shown. 82

B.2 The displacement field in a 2D plane strain mesh with a crack is shown. Magnified 5 times. . . . . . . . . . . . . . . . . . 83

B.3 The xx stress is shown in a 2D plane strain mesh with a crack. . . . . 84

B.4 The computed material force vectors near the crack tip in a 2D plane strain mesh. . . . . . . . . . . . . . . . . . 85

B.5 Plot of the stress vs distance from the crack tip down in the plane of the crack. . . . . . . . . . . . . . . . . 86

B.6 Plot of the displacement vs distance from the crack tip to the distance of one element in the crack opening direction. . . . . . . . . . . . . 87

C.1 The displacement field in a 2D plane strain mesh with a crack is shown. 90

C.2 The displacement field in a 2D plane strain mesh with a crack is shown. Magnified 5 times. . . . . . . . . . . . . . . . . . . 91

C.3 The xx stress is shown in a 2D plane strain mesh with a crack. . . . . 92

C.4 The computed material force vectors near the crack tip in a $2 \mathrm{D}$ plane strain mesh. . . . . . . . . . . . . . . . . . . . . . . . . . . . 93

C.5 Plot of the stress vs distance from the crack tip down in the plane of the crack. . . . . . . . . . . . . . . . . . . . . . 94

C.6 Plot of the displacement vs distance from the crack tip to the distance of one element in the crack opening direction. . . . . . . . . . . 


\title{
List of Acronyms
}

\author{
BC Boundary Condition \\ CTOA Crack Tip Opening Angle \\ CTOD Crack Tip Opening Displacement \\ EPFM Elastic Plastic Fracture Mechanics \\ FEA Finite Element Analysis \\ FEM Finite Element Method \\ GUI Graphical User Interface \\ LEFM Linear Elastic Fracture Mechanics \\ S-N Stress-Number of Cycles \\ SIF Stress Intensity Factor \\ WIC Welding Institute of Canada
}




\section{Chapter 1}

\section{Introduction}

\subsection{Background}

In the engineering world, the two most common and principal methods of joining metal parts are welding and bolting. Considerations of aesthetic appearance as well as the limited shear strength of bolts and resulting low endurance limit the application of bolted joints in industry. In case of welding, the parts are joined together with inter-atomic metallic bonds that ultimately form the weld joint. In many design situations, welded joints are considered better than bolted ones because component parts have nearly the same structural composition as the base material and the strength is generally equal to or even higher than that of the constituent parts [1].

The critical area of a steel structure or a component is a welded joint. Material properties at joints, such as ductility, ultimate strength, true stress vs strain relationship, vary from one region to another around the weld. In fact, almost all failures of welded structures occur because of the problems with welded joints. Failure depends on the materials that are used, type of the environment and operating conditions. The failure can be nucleated when the material approaches the limit of its strength, which can cause fracture. The most common failure of welded structures is due to fatigue which accounts for about $90 \%$ of failures [2]. Fatigue is a failure, which occurs 
in structures when they are subjected to fluctuating and dynamic stresses less than the yield stress of the material [3]. This type of failure can initiate at a stress level considerably lower than the yield or tensile strength for a static load. Fatigue nucleates cracks, that usually start at the surface. Once the crack is nucleated, it slowly grows by the virtue of a repeated cyclic load. This leads to a progressive damage on a reduced cross sectional area.

The loss of money or life when a structure fails due to fatigue failure can be large. The necessity for research to improve the longevity and reliability of the welded joints is therefore important. During the time of technical progress and computerization of the world, the use of finite element method has played a large role in engineering. This tool can help to assess the stresses caused by welding and in-service loading, as well as estimate the fatigue life of the structure, prior to its actual failure. Another positive side of computational weld mechanics for manufacturing is its time and money saving aspect.

According to Jonsson (2011), "Today, most programs, using finite element analysis can easily calculate any stress field, but the stress intensity at the crack tip requires more attention. Some programs can perform this type of analysis without putting much effort, but when it approaches to the calculation of automatic crack growth, the list of them narrows down only to a very few programs. It can be a complicated task for three dimensional analysis, since the mesh around the growing crack has to be remeshed for every step as well as moved in the right direction. To provide designers with a tool for building optimised lightweight structures, a future trend would be to build a software able to automatically calculate the crack path in a general three dimensional structure without having to put a lot of work into the task, personal or computational" [4]. 


\section{$1.2 \quad$ Research Strategy}

This thesis adopts the following research strategy.

1. An 3D transient non-linear analysis is done of welding a structure to compute the final state after welding. Instead of welding, this analysis could be for any manufacturing process that is capable of the computing the final state such as deformation, microstructure and residual stress. Any solver that can output the final state of the manufacturing process could be used.

2. Then, a 3D space-time analysis of a structure is done with in-service loads. The initial state is the final state of the manufactured structure, system or component. In this thesis, this is a quasi-steady state analysis of a welded structure with in-service loads.

3. The first two analyses were macroscopic and assume the structure has no cracks. The third analysis chooses locations that are considered to have a high risk of cracks nucleating, growing and leading to failure. In this thesis, FEM Gauss points with the highest values of principal tensile stress are considered to be the points of highest risk. At chosen Gauss points considered to be high risk, an elliptical crack is created that is normal to the principal tensile stress and 2D plane strain FEM meshes are created at points on the crack tip curve that normal to the crack tip.

4. These plane strain FEM analyses of these 2D meshes are solved with boundary tractions imported from the neighbourhood of this point in the second stress analysis. The stress intensity is computed at the point that the $2 \mathrm{D}$ mesh intersects the crack curve.

5. The number of load cycles to grow the fatigue crack a specified incremental distance is computed by solving the Paris-Erdogan equation. Other rules to 
choose the points of high risk of nucleating cracks could be used and other attributes of the crack parameters such as length and width could be chosen.

6. In a design stage, this risk must be predicted and this strategy could be used to optimize the design and reduce the risk of failure. In a structure operating with in-service loads, the crack location and parameters might be observed from NDT measurements. In this case, the observed data could be used to specify the crack location, size, shape and this strategy could be used to estimate the remaining life of the structure and to optimize the location and frequency of NDT tests.

7. Each $2 \mathrm{D}$ plane strain problem solve takes less than a minute of CPU time to solve with almost no effort to reduce CPU time. The solves are independent and thus trivially parallelizable. It is likely that CPU time could be reduced significantly. Parallel processing could be used to solve 1000's of potential crack locations for a given structure with given in-service loads.

One of the goals of this thesis is to demonstrate the capability to automate this procedure to minimize user time and user expertise in setting up the analyses and generating final reports.

\subsection{Directional scope of this study}

By investigation of works performed by other researchers, in the scope of fatigue analysis, it was feasible to notice potential expansions of scope and new areas of study that will be presented in this thesis. Some of the works will be described here.

Pyttel B., SchwerdtD., et al. [5] provides an overview of the current research on high cyclic fatigue failure $\left(N_{f}>10^{7}\right)$. The study provides a list of test facilities that are used for the research. Materials with typical S-N curves and affecting 
factors like residual stresses and notches are stated. The research tries to explain distinct failure mechanisms which occur while testing of the specimens. A double S-N curve is recommended for the representation of the fatigue behaviour, to take into account different failure mechanisms. One of the curves describes the surface fatigue strength behaviour and another one describes the volume fatigue strength behaviour. Recommendations about fatigue design of parts are given. Future scope of work about fatigue assessment of polymer components that are subjected to high number of loading cycles is established.

Myung Hyun Kima, Seong Min Kima, et al. [6] reveals a study of a fatigue strength assessment with the help of the hot spot stress method and the structural stress method, performed for a side shell connection of a container vessel. The study describes an approach for calculating the extrapolated hot spot stress for design purposes built on converged hot spot stresses. As a result, with the help of these two methods, hot spot stress approach and structural stress approach the fatigue strength at hot spot locations of a typical ship structure is predicted and compared. As a conclusion, it is mentioned that structural stress approach can be used as a viable alternative for the fatigue strength assessment of offshore structures and ships. Further directions of investigation related to fatigue strength of offshore structures are proposed.

Tveiten B. W. \& Moan T. [7] reviews the existing hot-spot stress methodology for plate structures; develops and verifies general and modern method for the structural stress extrapolation that can be used along with a hot-spot design S-N curve for ship structures made of aluminium. The research of Tveiten is intended to refine the analysis methodology built on the calculation of the structural stress and the selection of the appropriate S-N curves. The proposed method is used for determining the optimum location of points for stress extrapolation based on the asymptotic behaviour of the stresses adjacent to an idealized notch. Description of test rigs which were used for fatigue test is given. From the results of fatigue tests obtained in this research, 
appropriate $\mathrm{S}-\mathrm{N}$ curves along with a proposed extrapolation procedure can be used as a suitable choice for determining the structural stress and the fatigue assessment.

Maddox S. J. [8] gives a revision of methods, codes and standards related to the fatigue assessment of welded aluminium structures. Methods assessment for fatigue analysis of welded aluminium structures is made based on an estimation of the residual life of existing structures. Assessment of the methods includes information obtained from a literature search, but is primarily referenced to data used in recent fatigue design standards. Close attention is paid to recent fatigue data, acquired from structural components representative of actual structures. The research states that among the described fatigue assessment methods, the use of the nominal stress S-N curves is the most standardized and developed. The author assumes that in future the hot-spot stress approach will probably be the most valuable approach used for structural design.

Poutiainen I. \& Marquis G. [9] presents a method that is intended to expand the feasibility of structural stress method for fatigue analysis in welded structures. Conventional methods used for fatigue assessment are not able to take into account the effect of the weld size. Current study suggests a linearization of the local stress distribution through the thickness of the part in the plane of the weld toe, so the stress distribution is dependent on the size of the weld, part thickness and applied load. The paper covers equations used for calculating of the bilinear stress distribution. It is mentioned that the method was applied only to fully load-carrying welds, however it can also be used for partial load-carrying welds. The proposed stress distribution can be treated as a modification of the structural stress and it can be used in the same way as the conventional structural stress in the fatigue assessment of the welded structures. 


\subsection{Objectives}

This work is an attempt to evaluate the possibility and applicability of finite element method for fatigue analysis and crack growth prediction of welded structures. The aim of using the finite element method in fatigue analysis and design is to obtain a more precise estimation of the loads and stresses produced in the welded structures due to the welding process and in-service loadings. The fatigue analysis will be performed using VrSuite Software. VrSuite Software is a finite element analysis package, which can simulate welding processes, microstructural analysis and heat treatment. Different test cases composed of various geometries, meshes and materials are computed using VrSuite package to estimate the stress concentration and the stress intensity factors. Ultimately, the results of the stress intensity factor, computed by the finite element method, are compared to an analytical solutions used in linear-elastic fracture mechanics.

\subsection{Scope and Organization of the Thesis}

This thesis is divided into five chapters. Chapter 1 is an introduction to the thesis. It lists fatigue analysis studies that were conducted by other researchers. The basic understanding of factors that lead author to choose this topic, the general concept of the thesis, the outline of the research and objectives were also given in the Chapter 1. Chapter 2 gives insight into fracture mechanics theory, stress intensity factor calculation, crack initiation and propagation processes. Chapter 3 summarizes the theory used for fatigue life prediction which includes the fatigue analysis assessment methods. Chapter 4 includes the problem geometry definition and set-up, the analysis of the test cases based on the criteria of the stress concentration factor, the verification

of the VrSuite by comparing results obtained from the FEA with an analytical solutions 
and discussion of the obtained results. The conclusion and recommendations for the future work directions are given in Chapter 5. 


\section{Chapter 2}

\section{Fracture mechanics theory}

\section{$2.1 \quad$ Fracture mechanics concept}

Since cracks cannot be entirely eliminated, and they can lower the strength of a product below its expected strength, it is crucial to have the capability to quantify and predict the lifetime of a cracked structure under service load conditions. Fracture mechanics is the primary tool to analyse fatigue failures and brittle fracture in components. The main principle of the fracture mechanics is to provide a method of assessing the maximum service loading condition the component with a given crack size can withstand. Fracture mechanics is used to provide information on the conditions that lead a particular structure to failure and can also be used as a preventive measure for the life assessment of future products that operate in the same conditions. Fracture mechanics depend upon material properties, stresses induced by applied loads, defect size, shape and location. The behaviour of cracks in fracture mechanics is based on a stress analysis in the vicinity of the crack. Depending on the plastic region in the vicinity of the crack tip, fracture mechanics can be divided into linear elastic fracture mechanics (LEFM) and elastic plastic fracture mechanics (EPFM).

LEFM is associated with a small amount of plastic deformation at the vicinity of the crack tip. The parameter, which characterizes behaviour of the crack in linear 
elastic fracture mechanics, is defined by the value of the stress intensity factor $(K)$. Structures, used for LEFM evaluation require a high level of tensile strength along with a relatively low level of fracture toughness. In turn, elastic plastic fracture mechanics is used in cases when the plastic zone at the crack tip is slightly more extensive. EPFM is commonly used for the lower-strength structures with higher level of fracture toughness [10].

\subsection{Linear elastic fracture mechanics}

Fracture mechanics consider three basic modes of loading for fracture propagation [11]:

- Mode I, opening mode, as seen in Figure 2.1 (a) is associated with loading which is symmetrical about the plane of the crack and tends to separate the crack faces in opposite directions. The direction of the loading in this mode is perpendicular to the crack plane. Mode I is the most common loading case that leads to brittle failure.

- Mode II, sliding or shear mode, as seen in Figure 2.1 (b) is associated with in-plane loading which is anti-symmetrical about the plane of the crack, so the crack surfaces slide over one another. The direction of the load in this mode is parallel to the crack plane and normal to the crack tip curve.

- Mode III, tearing mode, as seen in Figure 2.1 (c) is associated with out-of-plane shearing forces. The direction of the loading in this mode is parallel to the crack plane and parallel to the crack tip curve.

Since Mode I is considered the most common and important in the majority of cracking analysis of engineering structures, only Mode I will be considered in this thesis. Opening mode involves two types of loading conditions in its analysis, plane 


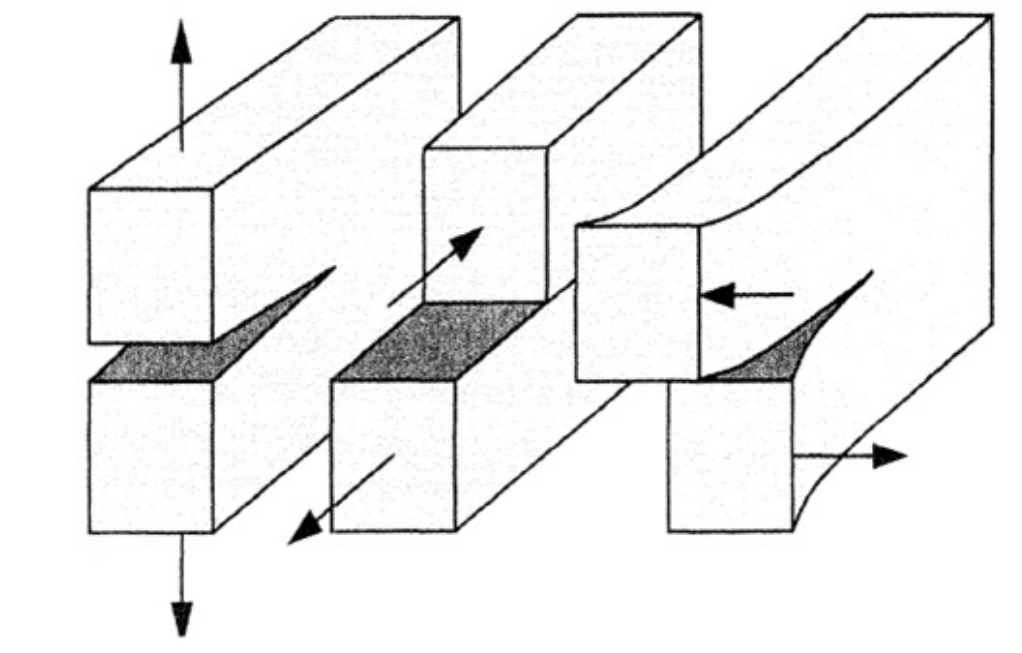

\section{$\begin{array}{lll}\text { (a) - Mode I } & \text { (b) - Mode II } & \text { (c) - Mode III }\end{array}$}

Figure 2.1: The three fracture modes [11].

stress and plane strain. The plain stress condition is inherent in thin plates, with stresses acting in its plane. In plane strain condition, in the XY plane, $\epsilon_{z}$ is constrained to be zero. It can be seen at the center of thick plates, stress components acting perpendicular to the plate thickness. Plane stress and plane strain conditions are illustrated in Figure 2.2.
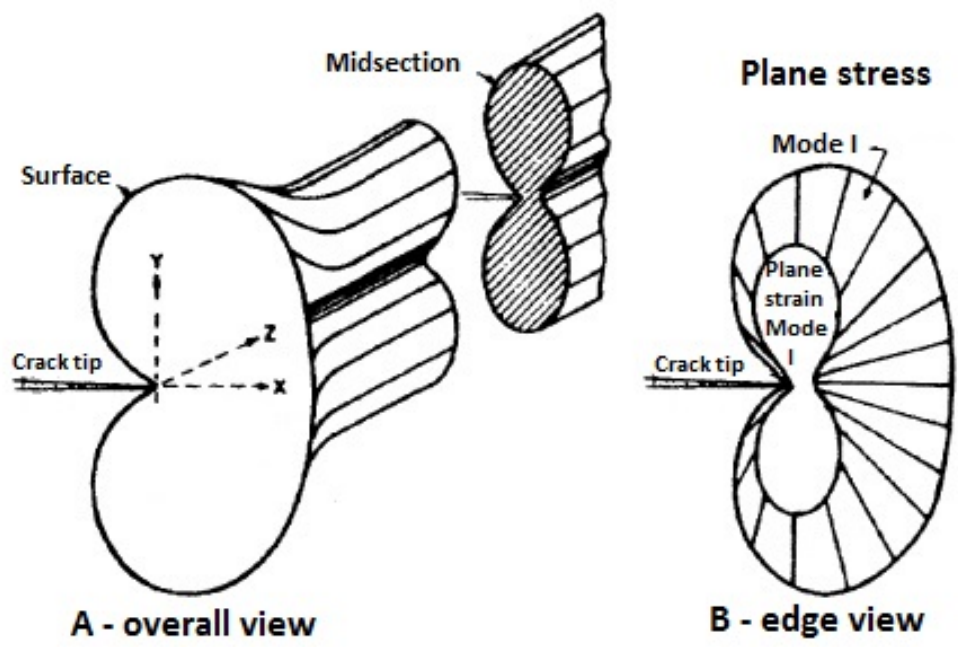

Figure 2.2: Plastic zone size [12]. 


\subsection{Crack initiation}

Crack initiation in metals can be considered as the formation of a void due to irreversible motion of dislocations under repeated deformation. The mechanism of void initiation in a ductile material can be characterized as homogeneous or heterogeneous. In case of a homogeneous crack nucleation, the voids initiate through the process of vacancy mitigation and dislocation interactions. In turn, the main characteristic of heterogeneous nucleation is interaction between dislocations and inclusions or other structural discontinuities [13]. Fatigue crack growth is usually slow and in many cases can be predicted by the use of fracture mechanics. Linear elastic fracture mechanics assumes quasi-static behaviour of the crack growth and a small plastic zone region in the vicinity of the crack tip.

\subsection{Crack tip plastic zone}

An important parameter that has to be taken into consideration while using linear elastic fracture mechanics is that the plastic zone at the crack tip must be small relative to the crack length and other size parameters of the part. Irwin noticed that if the size of the plastic zone is small compared to the size of the crack, then the amount of the energy for crack initiation can be computed from the elastic solution [14]. At the region beyond the plastic zone, linear elasticity equations apply [15]. Harlin \& Willis [16] provide an investigation which shows that singular elastic stress/full elastic stress ratio in LEFM is valid only in a range close to 1 and the plastic zone near the crack tip has to be sufficiently small (Figure 2.3). In order for the LEFM to be a good approximation, distance from the crack tip/crack length $(r / a)$ must be less than 0.05. As we go further away from the crack tip this approximation becomes less accurate [16]. 


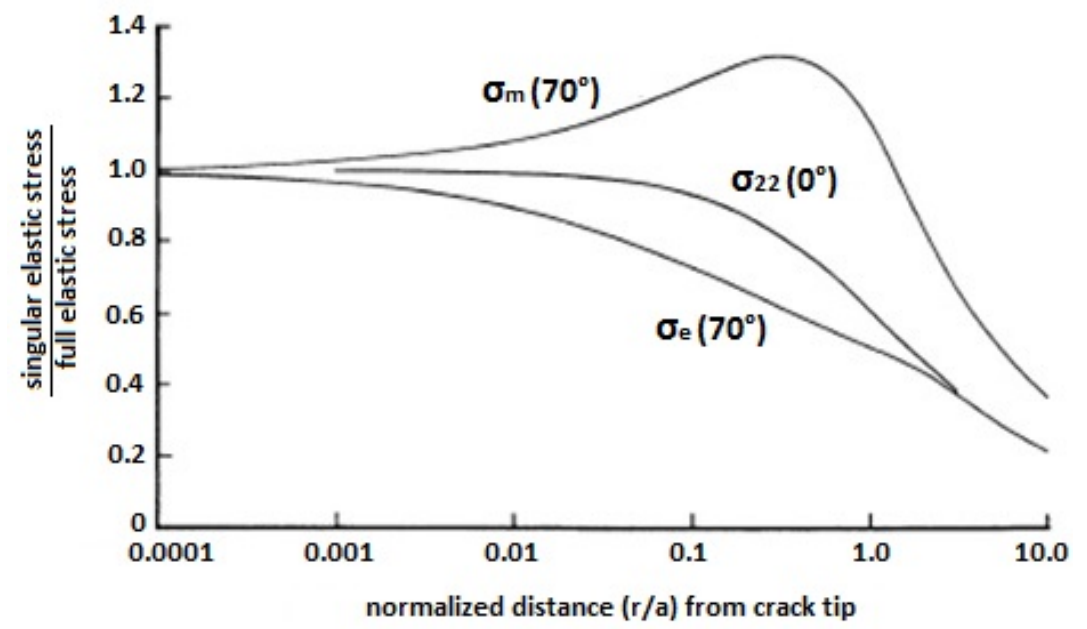

Figure 2.3: Plots of ratios of asymptotic forms to full stresses, as functions of r/a, adjacent to the tip of a buried crack in an elastic body, subjected to tensile stress. Where $\sigma_{22}$ at $\theta=0$ corresponds to the tensile stress normal to the crack [16].

\subsection{Stress intensity factor}

During operational conditions of welded structures, macroscopic flaws can be formed in a structure. Each of these flaws can be described as an infinitely sharp notch. In LEFM the measure of the crack propagation can be characterized by a single parameter called the stress intensity factor $K$. It characterizes the stress state in a sufficiently small region in front of the crack tip caused by residual or remote stresses and is illustrated in Figure 2.4. The value of this parameter depends on the crack size, loading condition, the geometrical configuration of a crack and construction where the crack is embedded [17]. The relationship between the stress state and the distance from the crack tip is shown in Figure 2.5.

When the value of the stress intensity factor $K$ reaches the value of the fracture toughness, $K_{C / I C}$, fracture in a structure will occur. The fracture toughness depends on the thickness of the specimen.

The stress intensity factor describes the crack tip state. Components of the strain, stress and displacement as a function of $r$ and $\theta$ in the vicinity of the crack tip can be 


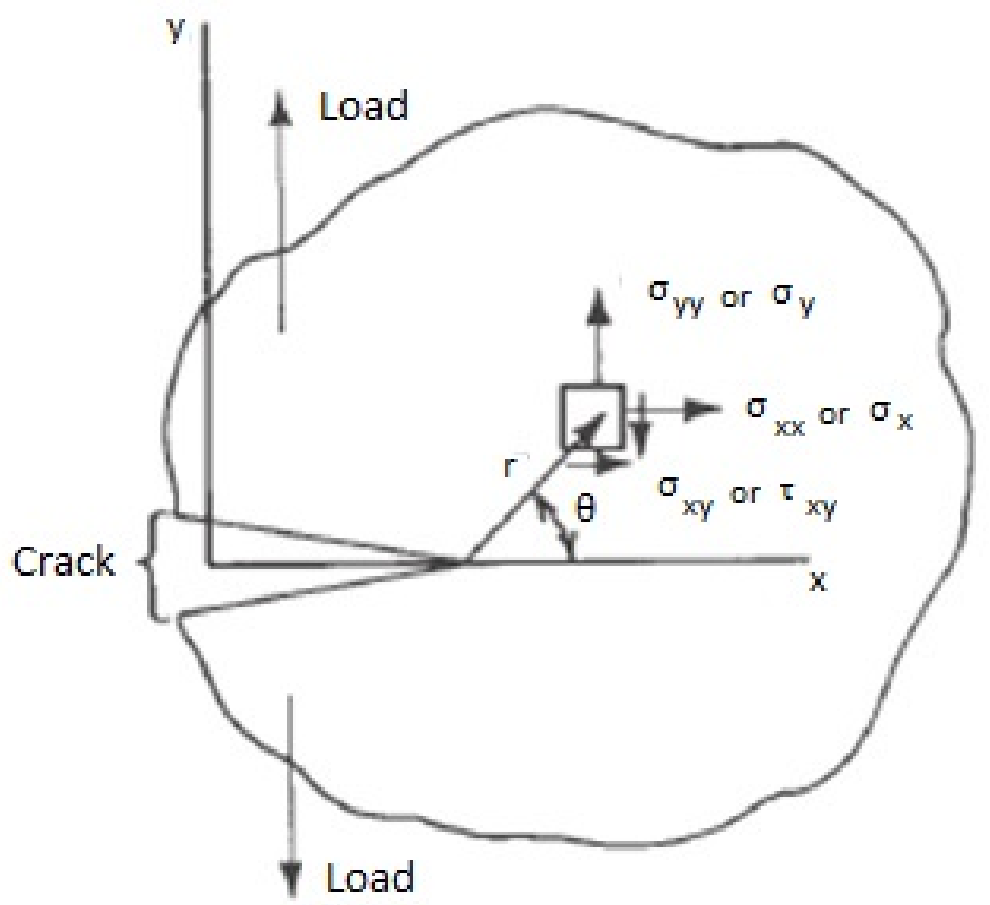

Figure 2.4: Polar and Cartesian coordinate systems at the crack tip that are used in LEFM [10].

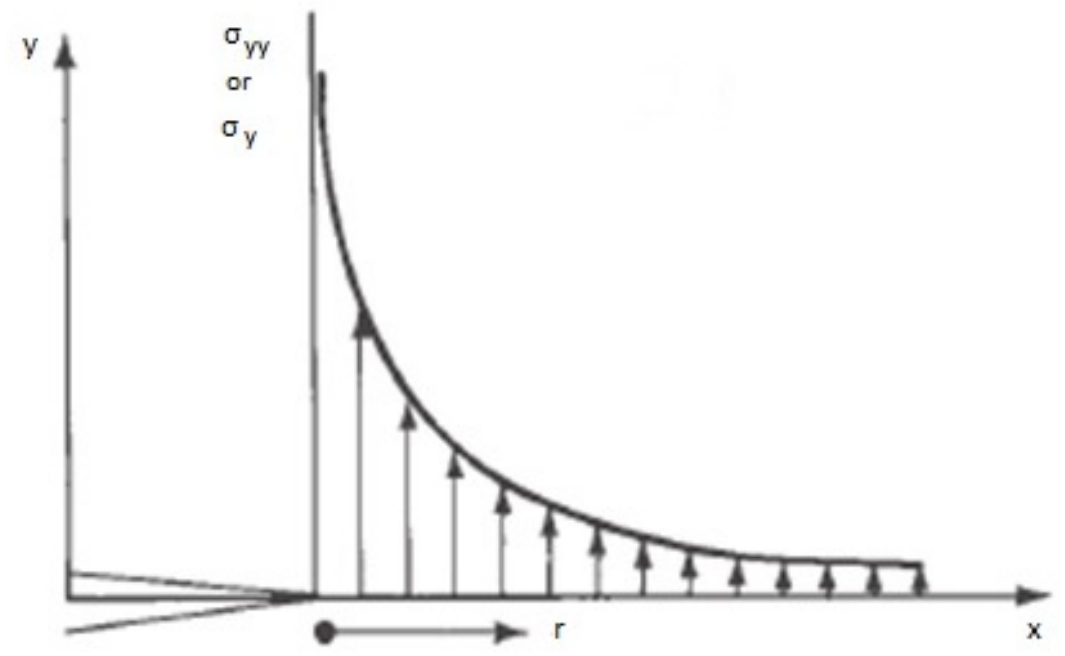

Figure 2.5: $\sigma_{y y}$ stress on the crack plane for a mode 1 loading of the crack [10]. 
computed by Equations 2.1 [11], if $K$ is known. Then, this information can be used to calculate the crack propagation rate during fatigue.

$$
\begin{aligned}
& \sigma_{x}(r, \theta)=\frac{K}{\sqrt{2 \pi r}} \cos \frac{\theta}{2}\left[1-\sin \frac{\theta}{2} \sin \frac{3 \theta}{2}\right] \\
& \sigma_{y}(r, \theta)=\frac{K}{\sqrt{2 \pi r}} \cos \frac{\theta}{2}\left[1+\sin \frac{\theta}{2} \sin \frac{3 \theta}{2}\right] \\
& \sigma_{x y}(r, \theta)=\frac{K}{\sqrt{2 \pi r}} \sin \frac{\theta}{2}\left[\cos \frac{\theta}{2} \cos \frac{3 \theta}{2}\right]
\end{aligned}
$$

Where $\sigma_{y}$, is a tensile loading (mode I) in $\mathrm{Y}$ direction, $\sigma_{x}$ is a stress in the plane of the crack, $\sigma_{x y}$ is a shear stress, $r$ is a distance from the crack tip, $K$ is a stress intensity factor and $\theta$ is an angle.

The term stress intensity factor was first introduced by Irwin in 1957. Since that time various methods to evaluate the value of the stress intensity have been developed. They can be classified into groups as: analytical methods, estimating methods, numerical solutions, etc. The solutions of $K$ for basic geometrical configurations and loading types can be found in reports or handbooks of Tada et al. 1985 or Rooke and Cartwright, $1976[18,19]$. In the case of a complex structure or inability to find the analytic equation to compute the stress intensity factor in a handbook, numerical methods are used. The finite element method is the most commonly used numerical method for the calculation of $K$.

Implementation of a finite element procedure to determine the stress intensity factor can occur in two different ways. One way is a direct method which is based on the utilization of the stress and displacement field of an object. Another possibility is an indirect method, which helps to determine $K$ from its relation with the J-integral and energy release rate [20]. 


\subsection{Crack propagation and Paris-Erdogan equa- tion}

Cracks in isotropic material usually start to form in the opening mode and tend to propagate in the direction perpendicular to the maximum tensile stress [21-23]. One of the crucial parts of fracture mechanics approach is calculation of remaining component life through prediction of the crack growth rate. The most common method which is used for prediction of fatigue crack propagation is the Paris-Erdogan law. It was long known that the rate of the crack propagation is not constant with time and increases with larger stress amplitudes and larger cracks [24]. Paris et al. (1961) was the first who proposed to use the stress intensity factor, developed by Irwin, for characterization of the rate of crack growth per load cycle [25]. He pointed out that fatigue crack growth increment per cycle relates to the increment in stress intensity factor. The Paris-Erdogan equation has the following form:

$$
\frac{d a}{d N}=C(\Delta K)^{m}
$$

The left side of the equation represents the crack growth per load cycle. It defines the relation between the increment in the crack length with the number of load cycles [24]. Where, $a$ is the length of the crack, $N$ is the number of load cycles, $C$ and $m$ are material constants, $\Delta K$ is the increment in stress intensity factor in a load cycle, that is expressed by the following equation:

$$
\Delta K=K_{\max }-K_{\min }
$$

Where $K_{\max }$ corresponds to the maximum value of stress intensity factor and $K_{\min }$ corresponds to the minimum value of the stress intensity factor in a load cycle.

In case of a known stress intensity factor, it is feasible to evaluate the fatigue crack 
growth in any crack configuration by using the Equation 2.2 .

The behaviour of fatigue crack propagation in metals can be assessed by the increment in stress intensity factor per load cycle $\Delta K[26]$.

Paris-Erdogan equation is limited to:

1. Large enough crack size.

2. Small enough plastic zone compared to the size of the crack.

3. Constant amplitude load cycles

Generally, the rate of fatigue crack propagation can be divided into three distinct regions as illustrated in Figure 2.6. In region I, where a threshold value of stress intensity factor range is low, propagation of the crack usually is considered not to occur under load fluctuation. This region is characterised by the small scale yielding and a small plastic zone. Region II is characterized by the linear relation between a crack growth rate and an increment in the stress intensity factor per load cycle. Crack length in this region is large relative to the plastic zone size. Most of the concepts that describe fatigue crack propagation behaviour in linear elastic fracture mechanics

are dealing with Region II. The Paris-Erdogan equation (Equation 2.2) is used to represent this region. Region III is characterized by high and unstable fatigue crack growth per cycle. The rate of the fatigue crack growth accelerates until the final failure occurs [26].

\subsection{Energy release rate}

The process of crack formation and propagation involves conversion of energy [28]. Griffith was first to see the importance of energy variation during crack growth in brittle solids [29]. He assumed that solids have a surface energy that must be 


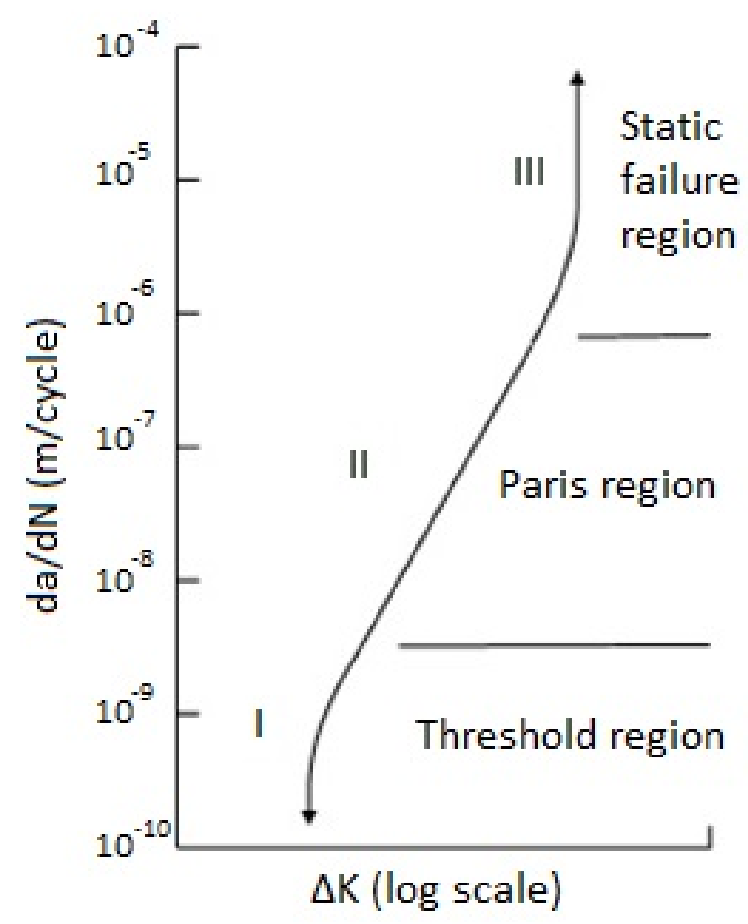

Figure 2.6: Three stages of fatigue crack growth [27].

compensated when propagating a given crack. Subsequently, the critical load level for a crack is found by the principle of minimum potential energy of elasticity with an extension that takes into account the surface energy of the solid [30].

The energy release rate $G$ can be an alternative to the stress intensity factor $K$ and is defined by a small-scale yielding. Since these parameters are interrelated, the value of one parameter can be easily determined from the other if one is known. When one of the arguments reaches its critical value, the other parameter also attains its critical values [31].

The strain energy release rate is defined as the change rate of potential energy of a cracked body with an increase in crack area for linear elastic materials. This parameter has a particular importance in fracture mechanics since the energy that has to be supplied to the crack tip for crack propagation must be balanced by the amount of energy absorbed due to the newly formed surfaces and other dissipative 
processes such as plasticity [31]. Energy release rate can be defined as:

$$
G=-\frac{d U}{d A}
$$

where, $U$ is the potential energy available for crack growth, and $A$ is the cracked area. The energy release rate failure criterion says that a crack will continue to propagate when the available $G$ is equal to or greater than a critical value of energy release rate $G_{c}$.

$$
G \geq G_{c}
$$

The value $G_{c}$ is a brittle fracture energy that is considered to be a material property and is independent of the applied loads and the body geometry [32].

In fracture mechanics J-integral and $G$ energy release rate are energy based parameters and directly related to stress intensity factor. Having either J-integral or energy release rate can significantly help in defining the $K$. Irwin established the following relation between fracture toughness and energy release rate [14]:

$$
G=\frac{K_{I C}^{2}}{H}
$$

where $H$ is the general elastic modulus:

$$
\begin{gathered}
H=E(\text { Plane stress }) \\
H=\frac{E}{1-\nu^{2}} \text { (Plane strain) }
\end{gathered}
$$

where $E$ is a Modulus of Elasticity, and $\nu$ is the Poison's ratio. 


\subsection{Material force method}

The material force method is an essential feature for improving and verifying numerical results computed with the Finite Element software. This concept provides clear and physically proved parameters for fracture assessment and prediction of crack growth. The method is based on the works of Eshelby [33] who used it to describe forces that act on inhomogeneities and defects.

Fracture occurs when the crack driving force becomes sufficiently large and attains the material's fracture toughness value. Direction of the crack is opposite to the crack driving force at the crack tip. Material force always appears at different types of inclusions such as cracks, holes and slits in the structures. In case of FEA it will appear at constrained nodes and boundary nodes [34].

In case of elasto-plastic and linear elastic material, material force and J-integral are equivalent to the energy release rate. J-integral can be simply derived as a negative material force projected on the $2 \mathrm{D}$ crack plane in the tangent to the crack path direction $t[35]$.

$$
J=-F_{m a t} \cdot t
$$

Additional background on the theory of material forces is provided by the following quotation from Steinmann [36].

The behavior of most materials (including composites, polycrystals, granular media and soft tissues to name but a few) is known to be influenced or even to be determined by inhomogeneities which those continua exhibit at a microscopic level. In the past decades, considerable effort has thus been concentrated on the development of extended continuum theories which are designed to account for inherent microstructure in natural and engineering materials. Existing extended continuum theories include among others 
micromorphic and micropolar theories, generalized continuum formulations based on order parameters and higher gradient formulations in either internal variables, state variables or both. These approaches have, despite their generally disparate nature, one thing in common: they are based on what is known as the "spatial setting" of continuum mechanics (alternatively referred to as a "direct-motion based formulation").

However, it is also possible to take a different approach to the modeling of microstructured continua suggested in essence in the mid-1950s by Eshelby [37]: a material setting based continuum description which gives rise to driving forces resulting from the material rearrangement of inhomogeneities during the deformation of a solid-like continuum. These driving forces essentially represent the tendency of general material defects such as cracks, inclusions, phase boundaries, dislocations and the like to move relative to the ambient material and are hence called material forces or configurational forces. As such, they are complementing Newtonian forces (regarded as physical driving forces for positional changes relative to the ambient space) arising in the spatial setting. Material forces contribute to the so-called balance of pseudomomentum, where they are balanced with Eshelby's stress tensor and the vector of pseudomomentum.

Examples of material forces are the PeachKoehler force acting on a dislocation in a defective crystal, and, in the context of elastic fracture mechanics, a vector-valued generalization of the classical J-integral, integrating the normal projection of the Eshelby stress over a surface enclosing the crack tip, namely

$$
J=\lim _{\partial V_{0}^{r} \rightarrow 0} \int_{\partial V_{0}^{r}} \sum \cdot N d a_{0}
$$


Here, $\sum$ is the Eshelby stress tensor and $N$ is the outward unit normal vector on the regular part of the boundary, $\partial V_{0}^{r}$.

The numerical analysis of material setting based continuum theories has become known as the material force method. An appealing feature of the latter is a straightforward and computationally cheap algorithmic determination of the material forces: it reduces to a post-processing step performed after the solution of the spatial motion problem has been determined and requires no additional data structures in addition to the usual finite element structures.

\subsection{Calculation of the SIF from the Crack Tip Opening Angle near the crack tip}

When the material force vector result is not clear (i.e. the vector of the maximum material force points not in the direction of the crack path), the value of the stress intensity factor for a linear elastic material behaviour can be computed from the CTOA on the crack faces near the crack tip by the use of the following equation [38]:

$$
K_{I}=\frac{2 S}{k+1} \sqrt{\frac{\pi}{2 l}}\left[4\left(u_{b 2}-u_{b 1}\right)-\left(u_{c 2}-u_{c 1}\right)\right]
$$

where: $K_{I}$ is the stress intensity factor for mode I, $S$ is the shear modulus, $l$ is the distance from the crack tip to the points $C_{2}$ and $C_{1}, u_{b 2}$ and $u_{b 1}$ are top displacement and bottom displacement values of the crack faces taken at points $B_{2}$ and $B_{1}, u_{c 2}$ and $u_{c 1}$ are top displacement and bottom displacement values of the crack faces taken at the points $C_{2}$ and $C_{1}, k$ is defined as:

$$
k=(3-4 \nu)(\text { Plane srain) }
$$




$$
k=\frac{3-\nu}{1+\nu}(\text { Plane stress })
$$

where: $\nu$ is the Poison's ratio. The meaning of the parameters: $l, u_{b 2}, u_{b 1}, u_{c 2}, u_{c 1}$ can be observed in Figure 2.7.

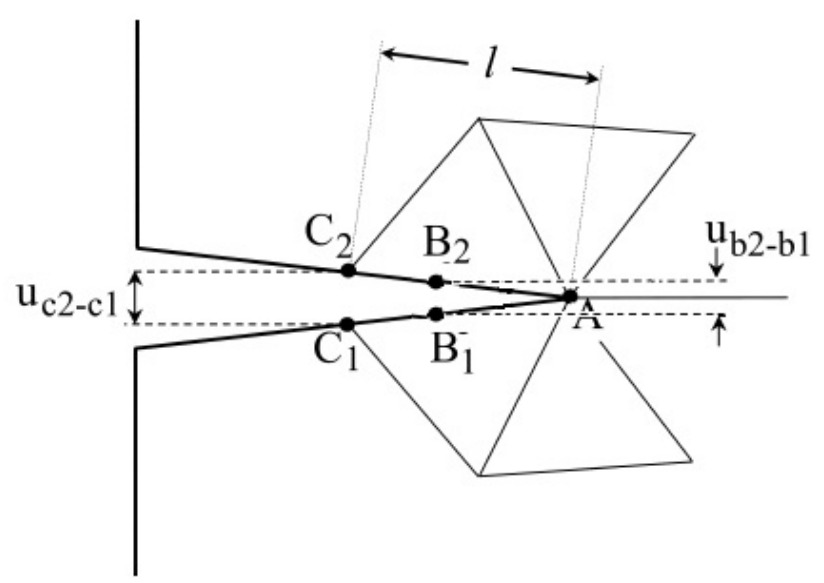

Figure 2.7: Evaluation of the SIF from the the crack tip opening angle (CTOA) [38]. 


\section{Chapter 3}

\section{Fatigue analysis assessment methods}

\subsection{Introduction}

Fatigue is a failure mechanism which occurs due to the action of dynamic and fluctuating stresses that lead to loss of the structure's nominal strength. Fatigue analysis of welded structures is of a high practical interest for cyclic loaded engineering structures such as bridges, cranes, vehicles, ships, offshore structures, etc. Fatigue in welded structures is a complicated process. Fatigue failures in welded structures mostly appear at the welds rather than in the base metal, even though the base metal may contain preliminary notches or cracks. The effects of the heating process and subsequent cooling of the weld as well as the fusion process create high residual stress concentrations in the weld with different geometrical parameters. Furthermore, welds usually contain cavities, pores, inclusions, undercuts, etc which act as stress raisers. A parameter that affects the fatigue behaviour of a weld joint is the residual stress and the distortion due to the welding process [39].

Fatigue failure always occurs at a stress level less than the tensile or yield strength for a static load. When the number of load cycles for a given level of stress intensity exceeds some tolerance threshold, microscopic cracks will initiate at the surface of the structure. Material damage starts in the crystalline structure and grows slowly by 
the cyclic plastic deformation as the stress cycles are repeated [40]. When the crack reaches a critical size due to fatigue the structure will suddenly fail.

Stress fluctuations due to the cyclic loading can have the following behaviour:

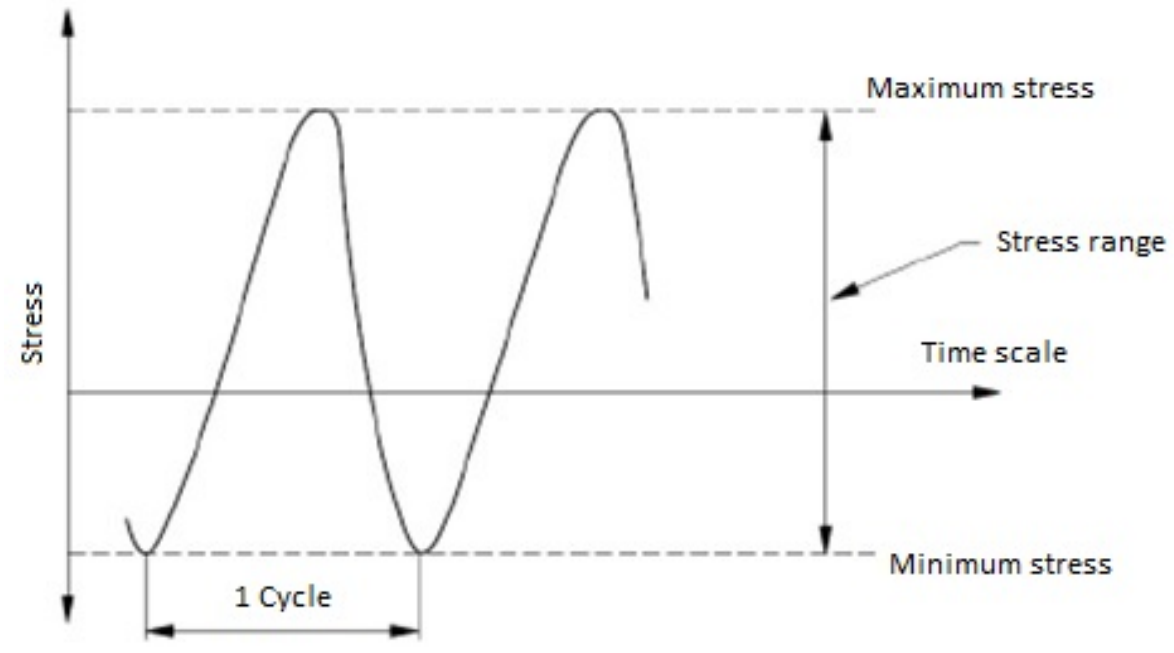

Figure 3.1: Stress variation in a fatigue load cycle [41].

Fatigue failures occur due to the aggregation effect of the damage caused by many repeated loading cycles. The figure above displays stress variation at a point due to load cycles. The most important components of the stress variation in fatigue analysis is the cyclic maximum and minimum stress value.

Four assessment methods have been introduced for estimating the fatigue life of steel structures under fatigue loading. These methods are based on the stresses, strains or stress intensity factors. These four methods can be divided into two groups: global and local methods. The first group contains only one method, which is the nominal stress method. The second group includes local methods and consists of the effective notch stress method, the structural hot spot stress method and the crack propagation method that is based on the principles of linear elastic fracture mechanics [42].

Fatigue cracking of cyclically loaded weld structures often starts at the weld toe, since weld toes can act as stress concentrators due to their geometry. To be able to predict local stresses at the weld toe, the weld geometry, in particular the radius and 
angle of a toe must be taken into account.

\subsection{Properties of S-N curve}

Generally, fatigue assessment tests can be divided into two types. The first type focuses on the nominal stress which cause fatigue failures for a definite number of load cycles. Results of such tests are usually represented by the plot of stress $\mathrm{S}$ on the $\mathrm{Y}$-axis against the number of cycles to failure $\mathrm{N}$ on $\mathrm{X}$-axis. This plot is named as $\mathrm{S}-\mathrm{N}$ curve, where $\mathrm{N}$ is generally plotted on a log scale as can be seen in Figure 3.2 [43].

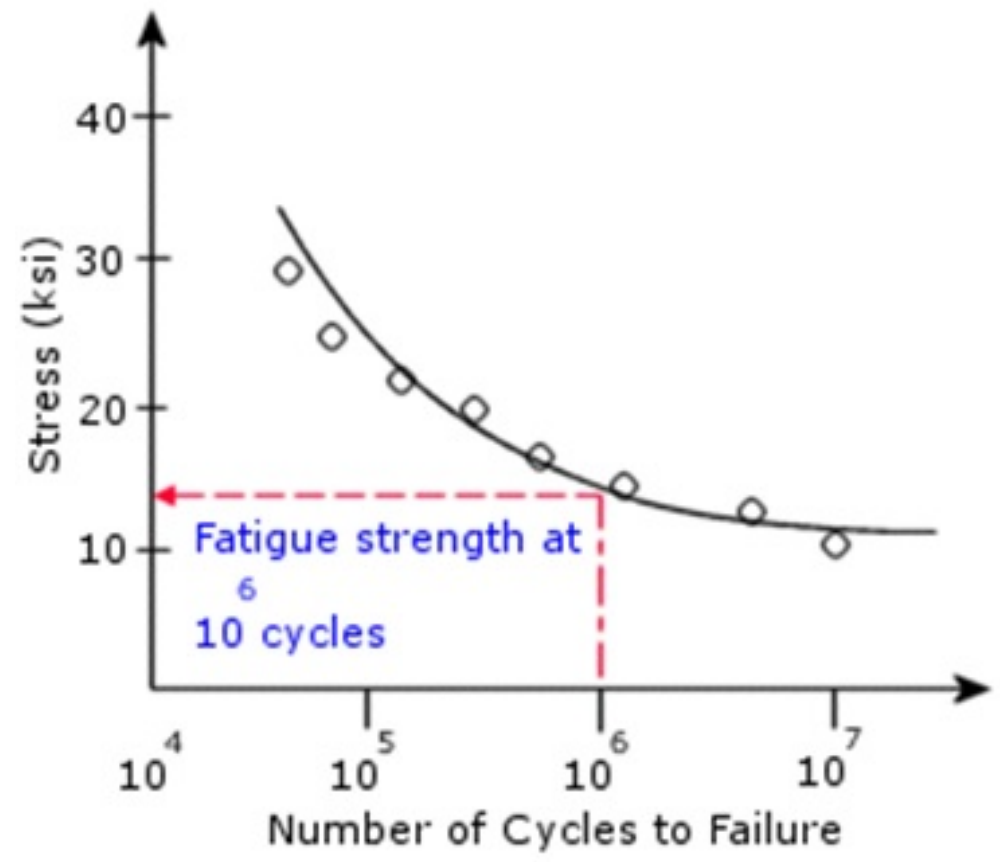

Figure 3.2: Typical S-N Curve [43].

\subsubsection{Stress life}

Data for such tests is obtained by cyclic loading of notched or smooth specimens until failure occurs. Conventionally, the first specimen is tested under a high peak stress. 
Further tests are performed with decreasing levels of stress until the specimens do not fail in a given number of cycles. Usually the maximum number of cycles are equal to $10^{7}$. Fatigue threshold stress is determined as the maximum stress at which run-out appears [44].

\subsubsection{Strain life}

Another type of fatigue test is the cyclic strain controlled test. While operating this test the strain amplitude is kept constant during the cyclic loading. This type of test is more typical for loading found during thermal cycling, wherein the specimen contracts and expands due to oscillation in the operating temperature [43]. 


\section{Chapter 4}

\section{Finite element analysis}

\subsection{Problem analysis}

\subsubsection{Two-dimensional stress analysis of a plate with a hole}

The following analysis evaluates accuracy and correspondence of the stress state of two-dimensional objects done by a finite element analysis with analytical solutions. The plate has a central hole and is subjected to tensile loading. The current analysis:

- Compares the maximum stress in the structure to the one derived from the analytical solution.

- Compares the calculated direction and magnitude of the material force vector to the one obtained from the literature.

\subsubsection{Determination of the maximum stress}

The analysis consists of a two dimensional plate with a hole in its center. Material that is used for this analysis is BABAnderson steel. The mesh type is 4 node quads. A tensile load is applied to the opposite sides of the plate. The plate has the following parameters: $W=0.1143 \mathrm{~m}, r=0.003175 \mathrm{~m}$ and an applied tensile load, $\sigma_{t}=3.78$ MPa. An example of the problem geometry can be seen on the Figure 4.1. 


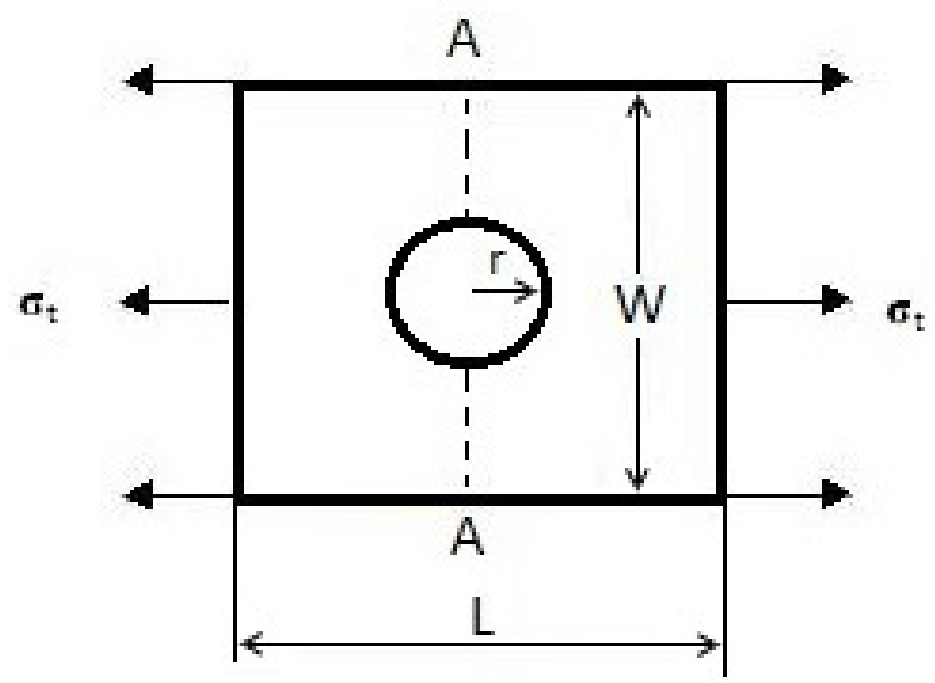

Figure 4.1: Two-dimensional model of the plate with following parameters: $W=$ $0.1143 \mathrm{~m}, L=0.1143 \mathrm{~m}, r=0.003175 \mathrm{~m}$.

The value of the analytical solution for the maximum stress $\sigma_{\max }$ at the boundary of the hole is taken from the paper [45]. The following equation is used to predict the maximum stress in the structure:

$$
\sigma_{\max }=K_{t} \sigma_{\text {nom }}
$$

where $K_{t}$ is the stress concentration factor and $\sigma_{n o m}$ is the nominal stress.

Stress concentration factor, $K_{t}$ can be derived from the equation below. The following equation can be used for a plate in tension with a hole that has a finite width. If the width is infinite the $K_{t}$ is equal to 3.0 [45].

$$
K_{t}=3.00-3.13\left(\frac{2 r}{W}\right)+3.66\left(\frac{2 r}{W}\right)^{2}-1.53\left(\frac{2 r}{W}\right)^{3}
$$

Nominal stress $\sigma_{\text {nom }}$ is determined along cross-section A-A as the mean tensile 
stress by the following equation:

$$
\sigma_{n o m}=\frac{W}{W-2 r} \sigma_{t}
$$

From the analytical equations above, the following results are obtained: nominal stress $\sigma_{\text {nom }}=4.0 \mathrm{MPa}$, stress concentration factor $K_{t}=2.8$ and maximum stress $\sigma_{\max }=11.4 \mathrm{MPa}$.

The finite element analysis consists of a two dimensional stress analysis of the plate with a hole that has the same geometry as the case for an analytical solution. A plane strain condition is applied. In order to have accurate results for the analysis, the mesh around the hole was refined. The element size of the refined mesh is $0.7 \mathrm{~mm}$. An example of the mesh can be seen on the Figure 4.2 .

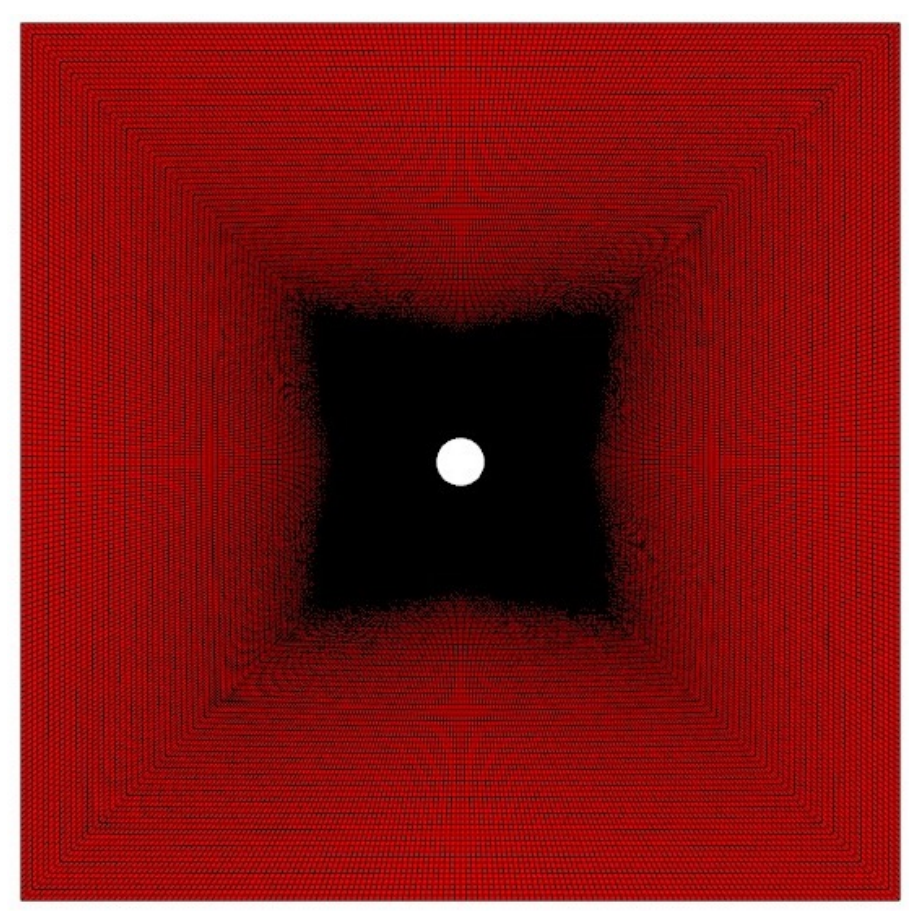

Figure 4.2: Mesh used for FEA analysis.

The tensile load applied to both sides of the plate with a hole causes tension stresses at the vertical axis, and compressive stresses at the horizontal axis. The result 
of the stress analysis is shown in Figure 4.3. The result shows that the maximum stress at the boundary of the hole is $\sigma_{\max }=11.9 \mathrm{MPa}$. By comparing the results acquired from the FEA analysis to results from the analytical solutions, it can be seen that the results for the maximum stress are consistent with the analytical solution, the difference is less than $5 \%$.

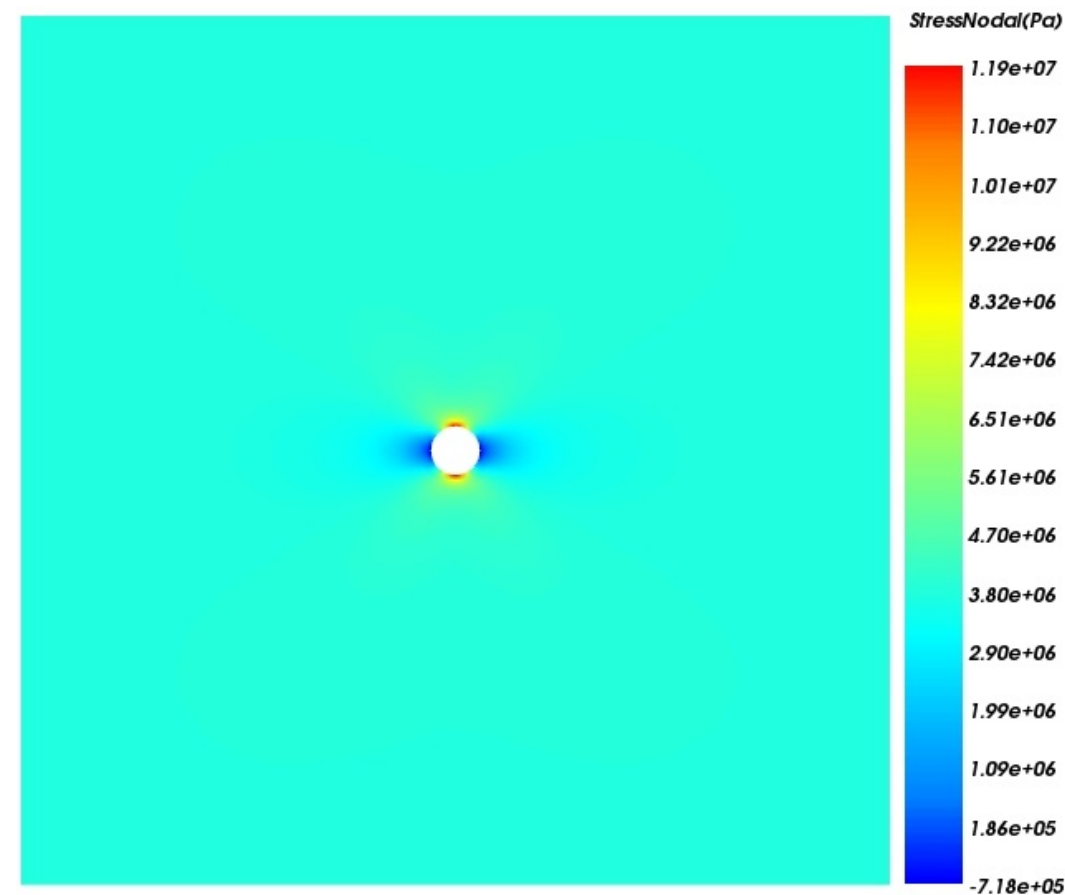

Figure 4.3: The result of the FEA stress analysis on a plate with a hole.

\subsubsection{Determination of the material force vectors}

The geometry which includes obtaining result of the material force vectors consists of a two dimensional plate with a hole in the center of it. Material that is used for this analysis is BABAnderson steel. The mesh type is 4 node quads. Displacement is prescribed to the top side of the plate in vertical direction and the rigid body mode constraints are applied to the bottom side. The whole structure has parameters: $W=10 \mathrm{~mm}, L=10 \mathrm{~mm}, d=4 \mathrm{~mm}$ and an applied displacement, $S=0,00015 \mathrm{~mm}$. An example of the problem geometry can be seen in the Figure 4.4 (a). The solution 
of this problem, which is taken from the paper [46], is displayed on the Figure 4.4 (b) and is compared later to the solution obtained using FEA.

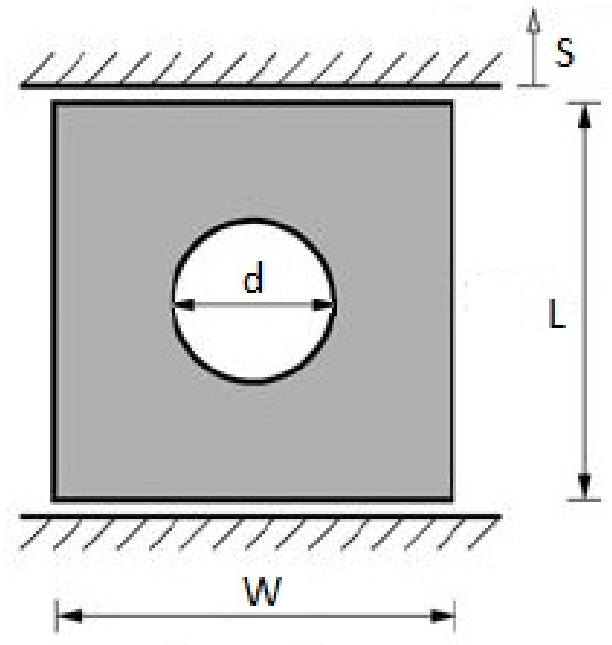

a)

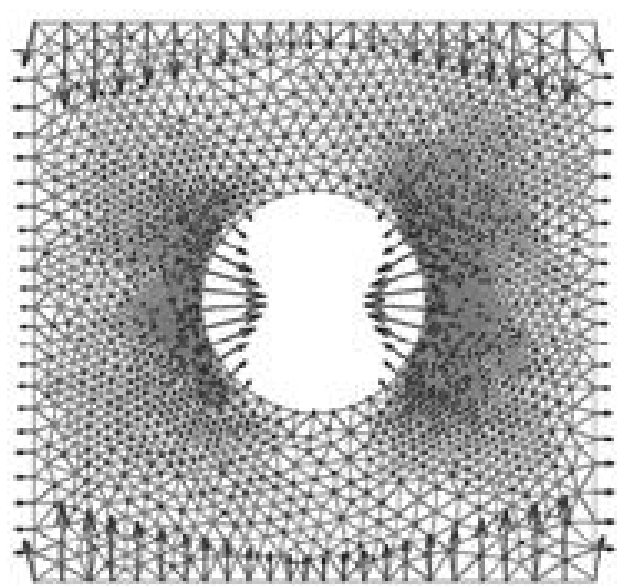

b)

Figure 4.4: a) Two-dimensional model of a plate with a hole and b) the result of the material force vectors taken from the literature [46].

FEA analysis using VrWeld was done with the same parameters. The FEM mesh that was used for the analysis can be seen in the Figure 4.5. The material force vectors obtained from the FEA analysis are compared to the analytical results and shown in the Figure 4.6. 


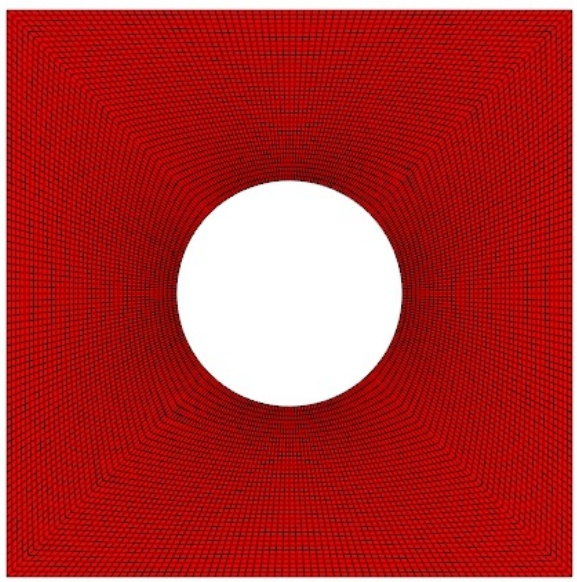

Figure 4.5: Two-dimensional plate with a hole for FEA analysis

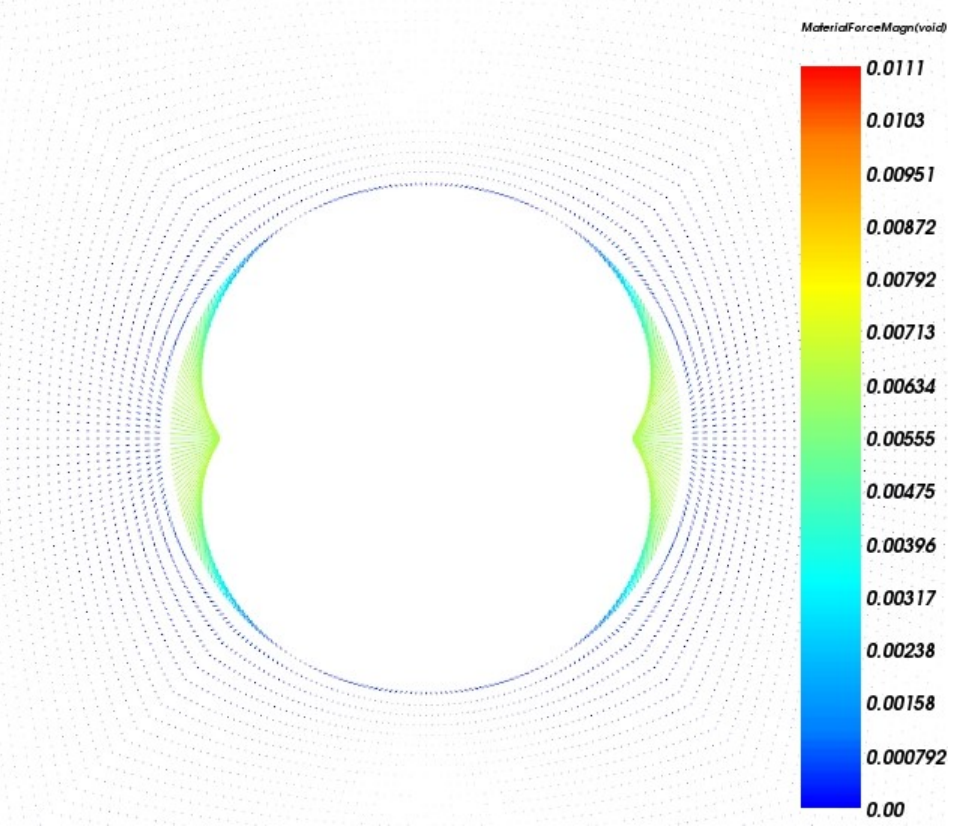

Figure 4.6: Material force vectors magnitude computed with VrSuite.

From the author's point of view [46] the hole can be considered as an inclusion with stiffness equal to zero. Since the plate is considered homogeneous, the material force vectors are expected to be zero in the regions where the FEA solution approaches the exact solution [34]. As can be seen from the results, the material force at the 
boundary of the plate goes to zero as may be expected for a FEM simulation with appropriately small error and fine mesh. Material force vectors inside the hole are non-zero and are in the direction of the large strain energy gradient due to the applied displacement.

\subsubsection{Stress analysis of an edge crack in a finite width sheet}

Edge crack analysis evaluates accuracy and correspondence of the stress state of two-dimensional objects done by finite element analysis with the analytical solutions. The aim of the current analysis is to:

- Compare the stress intensity factor obtained by VrSuite with the help of material force and J-integral to stress intensity factor evaluated from an analytical solution.

- Compare the result of the stress at the crack tip obtained by finite element analysis with an analytical solution derived by linear-elastic fracture mechanic.

- Determine the number of cycles to grow a fatigue crack along a prescribed crack path with the help of Paris-Erdogan equation.

\subsubsection{Procedure of a two dimensional manual crack analysis}

The main goal of this work is to perform fracture and fatigue analysis of the welded structures with the following strategy:

1. Perform a 3D stress analysis of an uncracked three dimensional object with applied boundary conditions and residual stress. Conduct a weld analysis to compute transient temperature, microstructure, stress, strain, displacement and internal variables.

2. Select a location for the crack in the $3 \mathrm{D}$ project. For the purpose of fatigue analysis, the set of Gauss points that have largest principal tensile stress in a fatigue load cycle are computed as candidate sites for possible fatigue crack 
nucleation. In terms of fracture analysis, possible fracture locations are chosen based on the Gauss points with highest principal stress and residual stresses.

3. In the current version, fracture and fatigue analysis are implemented for the surface cracks, therefore the Gauss points that have the highest principal stress values at the surface are selected. At the selected position, an elliptical crack is placed with its center at the Gauss point and a crack plane normal to the principal tensile stress. The width and depth of the elliptical crack are chosen to be independent parameters.

4. Furthermore, a two dimensional fine plane strain mesh that is perpendicular to the crack tip and normal to the crack surface is created. The position of the $2 \mathrm{D}$ plane strain mesh can have different angles relatively to the crack. This plane contains results of the stresses from the 3D analysis with no crack projected into the face of the crack.

5. In each $2 \mathrm{D}$ mesh created, as described above, stresses are mapped from the 3D object with no crack to the mid-point of the corresponding face element on the crack surface. Given the coordinates of the mid-point of face element on the crack surface, the outward normal of the mid-point of face element on the crack surface, the 3D stress tensor multiplies as a 3D matrix times the outward normal to compute the tractions acting on the mid-point of each face element on the crack surface. This traction is used to compute the Neumann boundary conditions of the tractions acting on the crack faces of elements. In addition Dirichlet boundary conditions are applied to constrain rigid body modes of the two dimensional plane to zero. At the two bottom corners, the y-displacement is constrained to zero and at the mid-point of the bottom edge, the x-displacement is constrained to zero. 
6. With this state and boundary conditions, the two dimensional plane strain analysis of the fine crack mesh for one point on the crack tip is performed.

7. A plane strain analysis determines the material force at the crack tip that is used for calculating of the J-integral and the stress intensity factor.

8. When the result of the material force vector is not clear, the value of the SIF is computed from the CTOA on the crack faces near the crack tip.

9. Once the value of the stress intensity factor associated with each point on the crack tip is known, it can be compared to the value of fracture toughness of the structure in terms of fracture mechanics analysis.

10. For the purpose of fatigue analysis, (i.e. calculating the number of cycles to grow for a crack), a crack with a new length has to be introduced. The location of the crack, boundary conditions and parameters of the crack analysis remain the same, as the crack length increases. This will allow to compute the increment of the stress intensity factor of a new crack.

11. From the increment in the stress intensity factor, the rate of crack growth per load cycle can be computed by solving the Paris-Erdogan equation.

12. An auto-report for fracture and fatigue analysis can be generated.

\subsubsection{Determination of the stress intensity factor}

The three dimensional plate, shown in Figure 4.7, with displacement applied to both top and bottom of the plate in the vertical direction was created. The width of the plate is $0.21 \mathrm{~m}$, the length is $0.62 \mathrm{~m}$ and the thickness is $0.001 \mathrm{~m}$. Material that is used for this analysis is BABAnderson steel. The 3D FEM mesh consists of 8 node brick elements and a mesh for a fine 2D crack analysis uses 4 node quads. A displacement of 
$0.00001 \mathrm{~m}$ is applied to the top and bottom sides and the z-displacement is constrained to zero at all nodes in the plate to enforce the plane strain condition in the plate. The result of the applied displacement in vertical direction can be seen in the Figure 4.7.

The dimensions of the 2D crack plate for analytical solution and FEA analysis were chosen to be the same to be able to compare results. A general view of the crack plane is shown in the Figure 4.9, where: $b=0.21 \mathrm{~m}, h=0.31 \mathrm{~m}, a=0.06 \mathrm{~m}$, $S=0.00001 \mathrm{~m}$.

After performing a stress analysis of the two dimensional plate, the following result of the displacement was obtained and is displayed in the Figure 4.8. As we can see from Figure $4.8 \mathrm{~b}$ ) during magnification of the displacement the crack is observed to open, indicating that the results from the 3D object are mapped correctly.
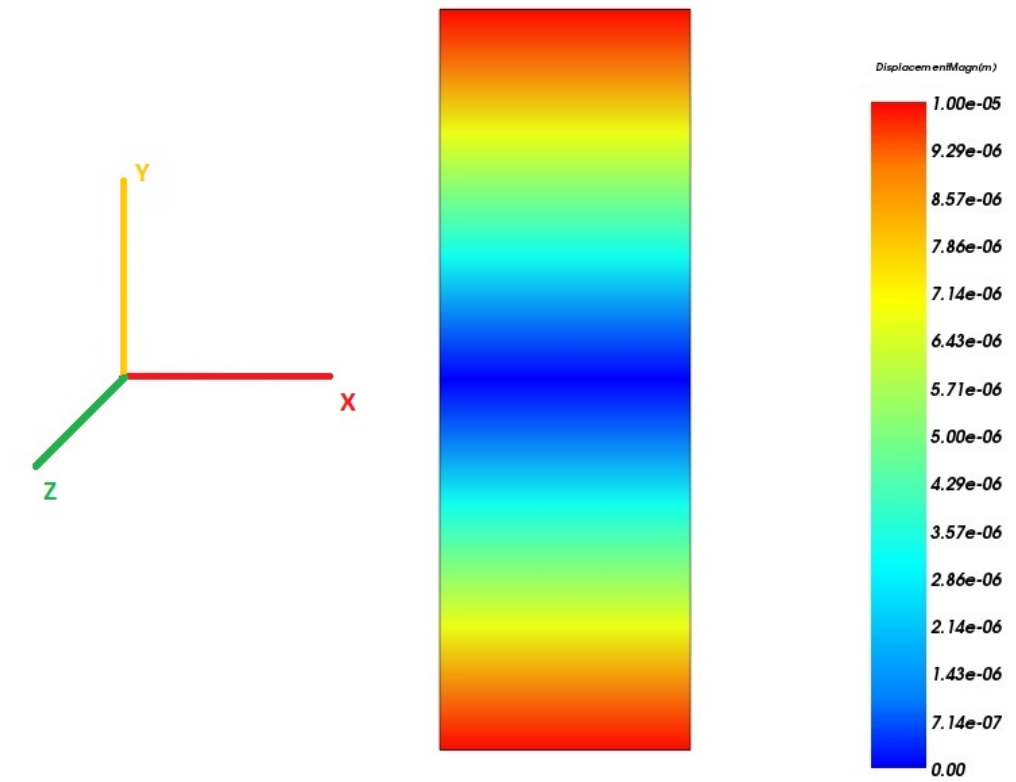

Figure 4.7: Displacement field in the 3D plate without crack with prescribed displacement BCs at the top and bottom ends of $0.00001 \mathrm{~m}$. 


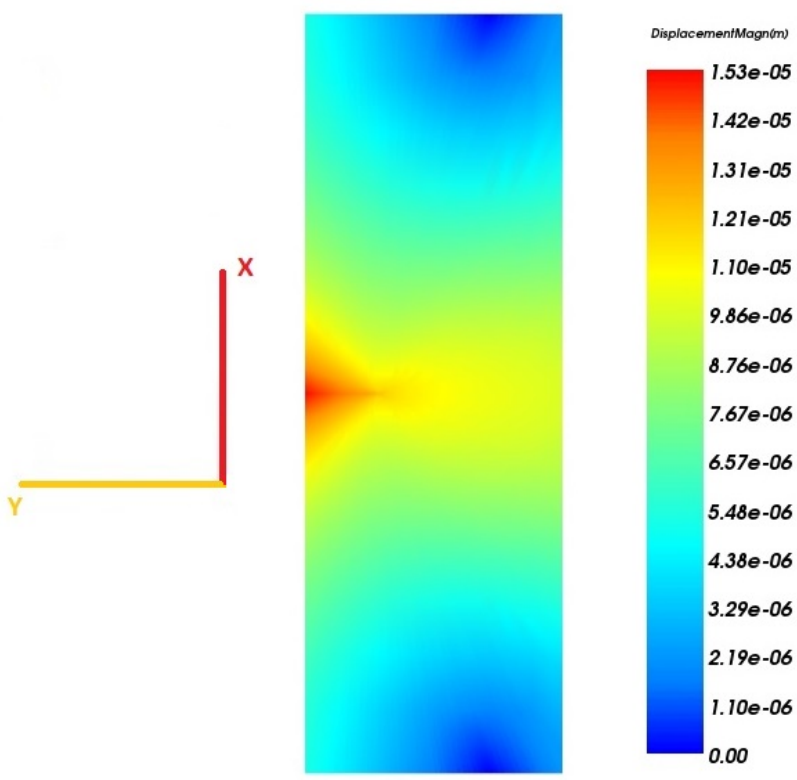

a)

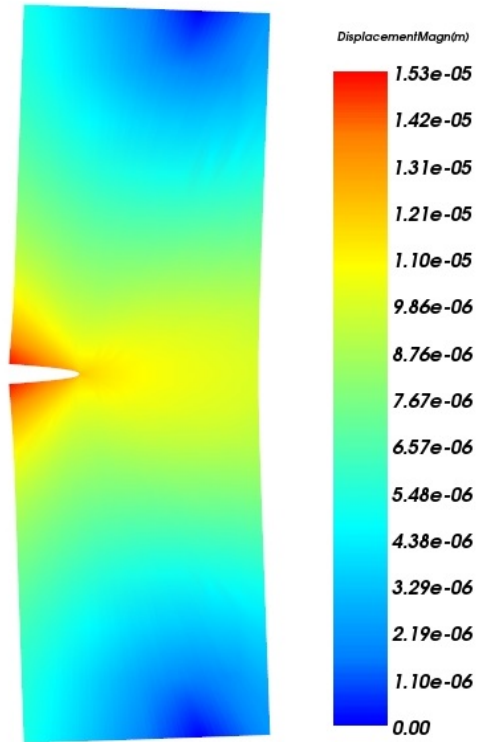

b)

Figure 4.8: a) Displacement in the 2D plate with a crack and b) displacement field magnified 1000 times in the 2D plate with a crack.

The analytical solution of stress intensity factor $K_{I}$ for an edge crack caused by mode I loading is obtained from the equation below [19].

$$
K_{I}=\sigma \sqrt{\pi a}\left[1.12-0.23\left(\frac{a}{b}\right)+10.6\left(\frac{a}{b}\right)^{2}-21.7\left(\frac{a}{b}\right)^{3}+30.4\left(\frac{a}{b}\right)^{4}\right]
$$

This equation is valid for ratios of $h / b \geq 1.0$ and $a / b \leq 0.6$. The applied stress for this equation can be simply derived from the displacement and is equal to 6.72 $\mathrm{MPa}$. The value of the analytical solution of stress intensity factor for this analysis is 4.71 $\operatorname{MPa} \sqrt{m}$ 


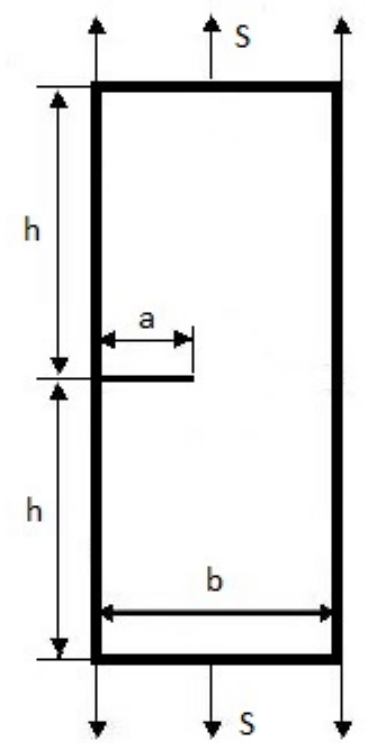

Figure 4.9: Two dimensional crack plane.

Stress intensity factor computed by VrSuite is evaluated with the help of the J-integral by using the equation below:

$$
K_{I}=\sqrt{\frac{J E}{1-\nu^{2}}}
$$

where J-integral is derived as the negative material force projected to the $2 \mathrm{D}$ crack plane which is tangent to the crack path direction [35].

$$
J=-F_{m a t} \cdot t
$$

The result of the material force solved by finite element analysis is displayed in the Figure 4.10. The material force vector with the maximum value starts at the crack tip and points in the direction opposite to the crack growth.

The computed stress intensity factor for an edge crack with the help of the equations above has a value 4.59 $\mathrm{MPa} \sqrt{m}$. The error between the analytical solution and the computed solution is only $2.5 \%$ which is considered a good agreement between 
analytical and FEA results.

The obtained value of the stress intensity factor can be compared to the known value of fracture toughness of the material, determined by the fracture toughness test, to assess the resistance of the material to fracture.

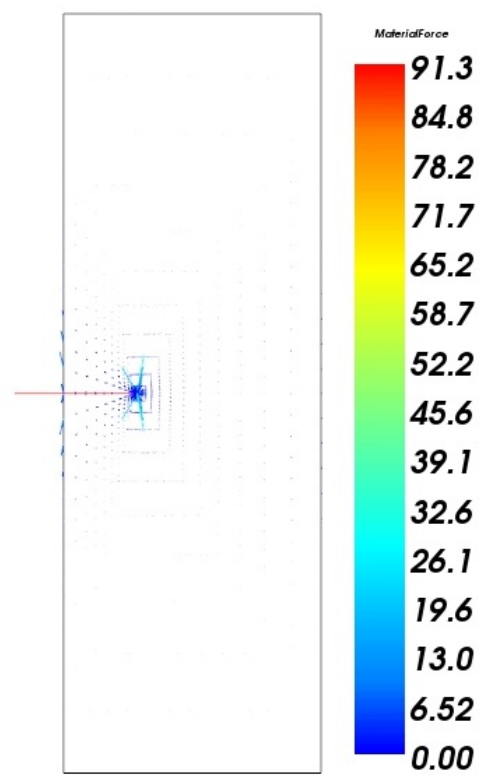

a)

91.3
84.8
78.2
71.7
65.2
58.7
52.2
45.6
39.1
32.6
26.1
19.6
13.0
6.52
0.00

0.00

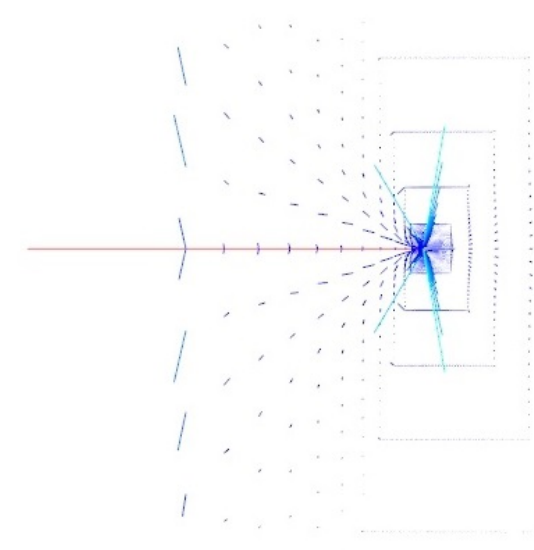

b)

Figure 4.10: a) Material force vectors are shown near the crack tip and b) zoomed in section showing the material force vectors near the crack tip. The vector shown in red is the material force vector acting on the crack tip.

\subsubsection{Comparison of the stress at the crack tip}

From Section 2.4 we know that analytical stress derived under LEFM assumptions is valid only for a certain ratio of the distance from the crack tip to crack length and this ratio of $r / a$ should be less than 0.05 . Since the size of the crack is $0.06 \mathrm{~m}$, the distance from the crack tip should not exceed the value of $0.003 \mathrm{~m}$.

The result of the computed nodal stress in xx direction with the help of FEA can be found in Figure 4.11, with the highest value of $189 \mathrm{MPa}$. By knowing the value of 
SIF for analytical solution of the stress under LEFM and using equation 2.1 we can derive the values of the stress away from the crack tip. The highest value of the stress for analytical solution is $187 \mathrm{MPa}$.

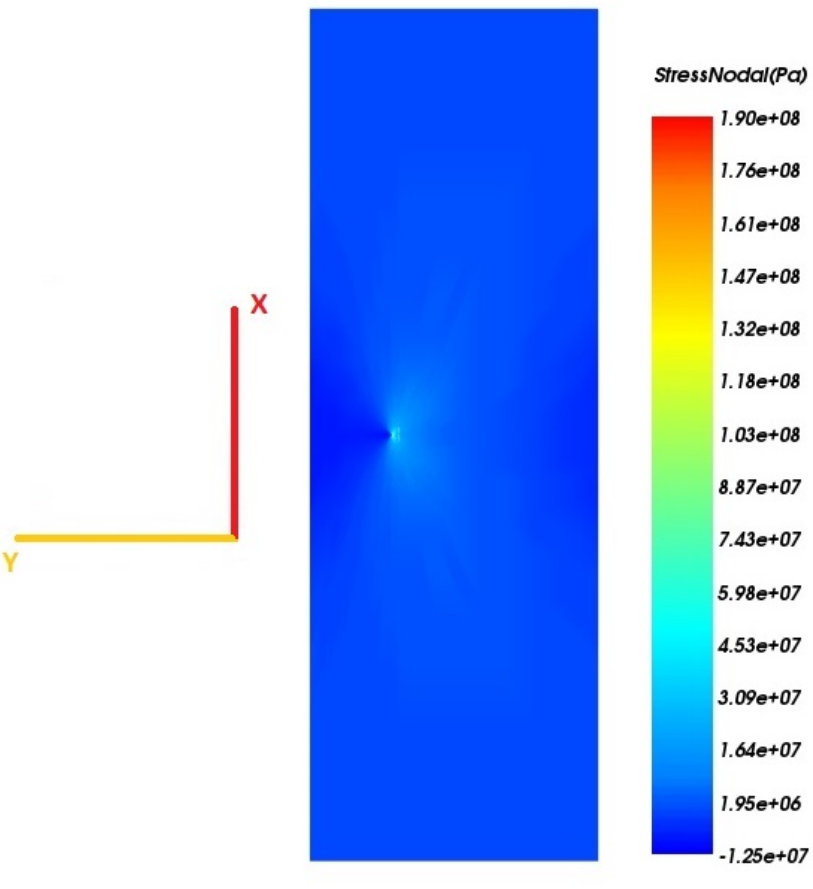

a)

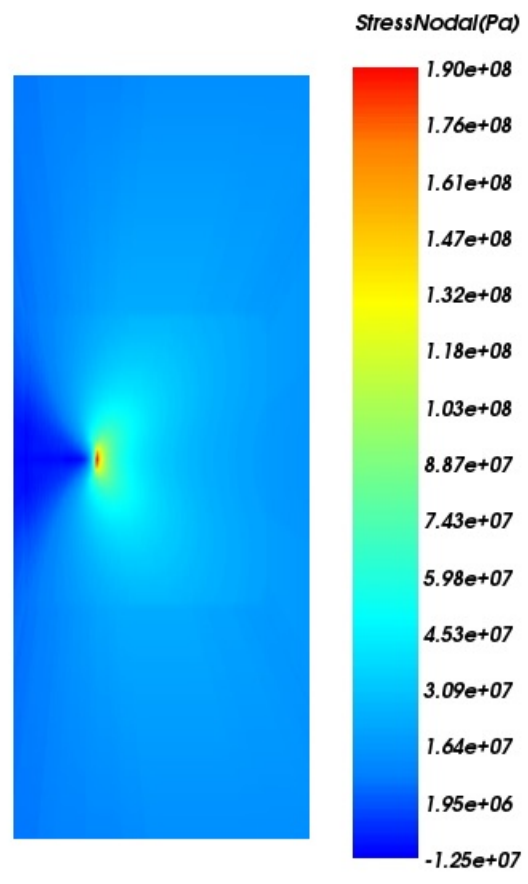

b)

Figure 4.11: a) The computed nodal stress in the xx direction for the 2D plate with the crack and b) zoomed in result of the nodal stress in the xx direction for the $2 \mathrm{D}$ plate with the crack.

To be able to compare results of the stress at the crack tip obtained by finite element analysis with the analytical solution derived by linear-elastic fracture mechanic the plot of the stress versus distance from the crack tip was made and displayed in Figure 4.12. It may be observed from the plot that the FEM results are consistent with the analytical solution. 


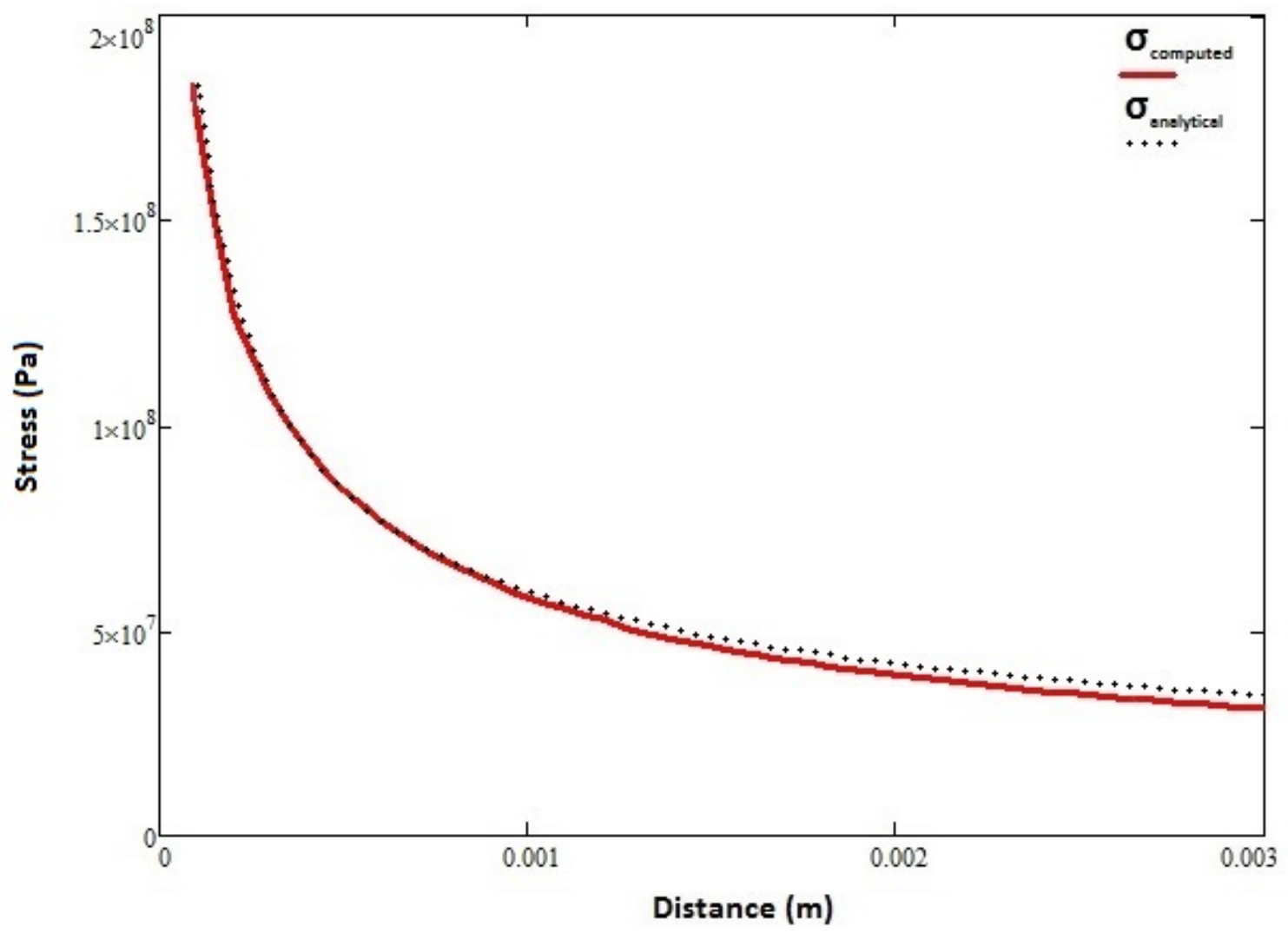

Figure 4.12: Comparison of the stresses at the crack tip computed by FEA with analytical solution derived by LEFM.

\subsubsection{Determination of the number of cycles for a fatigue crack growth}

The number of load cycles for fatigue crack growth can be determined from the

Paris-Erdogan equation (2.2). For this particular case the Paris-Erdogan equation can be integrated into the following form, which is more accurate than Equation (2.2) and is used for mode-I tests [47].

$$
\begin{gathered}
\Delta N=\Delta a C^{-1}(\Delta K)^{-m} \\
\Delta N=\frac{\Delta a\left(\left(\Delta K_{a-\Delta a}\right)^{1-m}-\left(\Delta K_{a}\right)^{1-m}\right)}{C\left(\Delta K_{a}-\Delta K_{a-\Delta a}\right)(m-1)}
\end{gathered}
$$


Where $\Delta a$ is an increment in crack length in the load cycles; $\Delta K_{a}$ equals to the value of $\Delta K$ when the crack length is $a ; \Delta K_{a-\Delta a}$ is equal to the value of $\Delta K$ when the crack length is $a-\Delta a$.

The following analysis was done with the same value of applied displacement, geometry and parameters as for calculation of the stress intensity factor. Initial crack length equal to $0.06 \mathrm{~m}$. The crack increment for this case is $0.01 \mathrm{~m}$. The crack was grown from the value of $0.06 \mathrm{~m}$ to $0.07 \mathrm{~m}$. The value of the stress intensity factor for the final crack is $5.84 \mathrm{MPa}$. The values of the Paris-Erdogan constants for the analysis are chosen to be $C=6.9 \times 10^{-12}(\mathrm{~m} /$ cycles $)(M P a \sqrt{m})^{-m}$ and $m=3.0$.

Equation 4.8 was used to determine that the number of cycles to grow the fatigue crack from $0.06 \mathrm{~m}$ to $0.07 \mathrm{~m}$ for the given material is equal to $10,508,142$ cycles.

\subsection{Manual fatigue analysis of the laser weld}

\subsubsection{Problem geometry}

Current analysis describes the steps of manual fatigue analysis of the laser weld with the help of VrSuite. The specimen geometry consists of two plates joined by laser welding. The material used is A13C steel. The 3D FEM mesh consists of 6 node triangular prism elements. The dimensions of the top plate are $40 \mathrm{~mm} \times 1.5 \mathrm{~mm} \times$ $20 \mathrm{~mm}$ and the dimensions of the bottom plate are $28 \mathrm{~mm} \times 3 \mathrm{~mm} \times 20 \mathrm{~mm}$. The geometry of the specimen is represented in the Figure 4.13. 


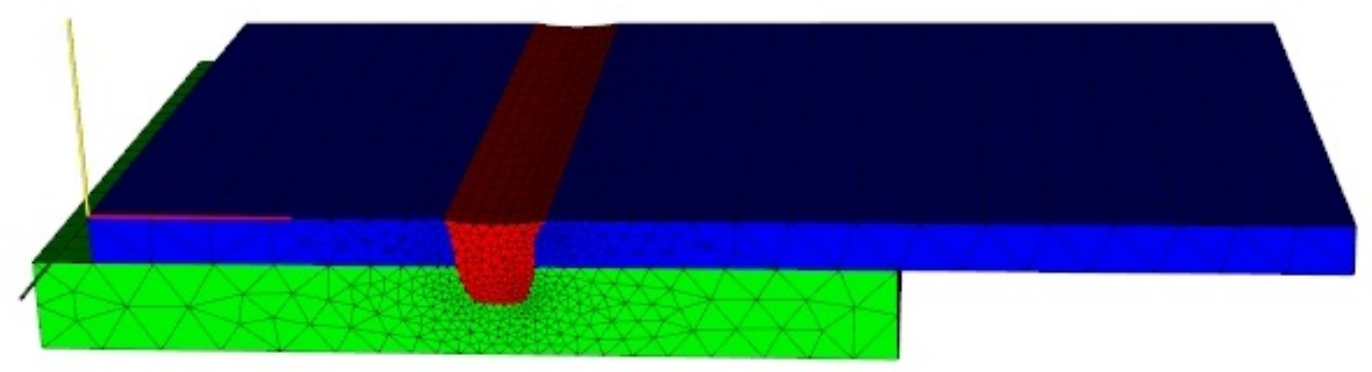

Figure 4.13: Geometry of the specimen. Triad axes: red is the $\mathrm{X}$ axis, yellow is the $\mathrm{Y}$ axis, green is the $\mathrm{Z}$ axis.

\subsubsection{Setup and analysis}

Once the mesh is created and the welding is simulated the following steps are done in order to manually analyse the laser weld specimen:

- A tensile load of $100 \mathrm{MPa}$ is applied to the top right and bottom left parts of the sample and rigid body modes are constrained. A weld analysis that computes transient temperature, microstructure and stress is performed.

- When the stress results are obtained, the Gauss point on the surface with the highest tensile principal stress is picked as a place where possible fatigue crack can initiate. The set of all Gauss points in the structure can be seen in Figure 4.14 (a). Figure 4.14 (b) shows a zoomed in region of the Gauss points for a narrow range of maximum values in order to guide the selection of the point on the surface with the maximum value of the tensile principal stress. It is 
assumed that the structure doesn't have a crack due to the welding and crack nucleates because of the residual stresses after welding and in-service loading of the specimen.

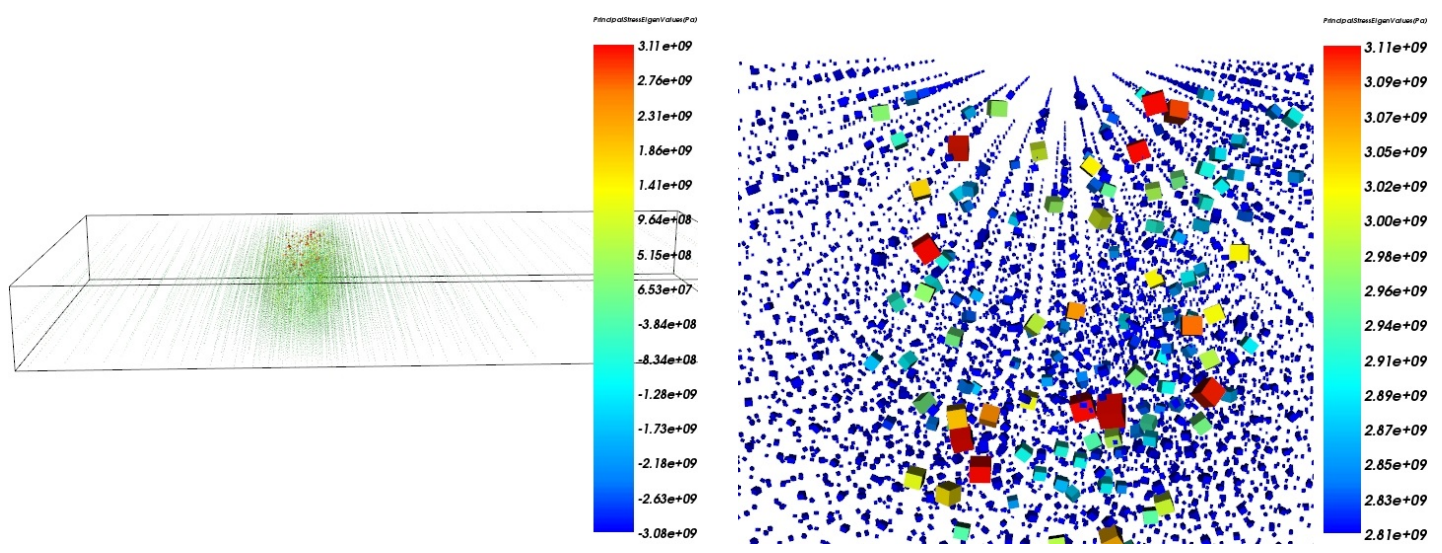

a)

b)

Figure 4.14: a) Result of the Gauss points that show a principal stress in the structure b) Zoomed in region of the Gauss points with narrow range of the maximum values.

- Once the node is picked, an elliptical crack is created with the center at the chosen Gauss point. The length of the ellipse for current analysis is set to 1.6 $\mathrm{mm}$ and the width is $1 \mathrm{~mm}$. The $2 \mathrm{D}$ crack plane is created normal to an elliptical crack and in plane with the applied tensile load. The crack length is set to 0.5 $\mathrm{mm}$. The mesh of an elliptical crack with perpendicular to it $2 \mathrm{D}$ crack plane is shown in Figure 4.15. 


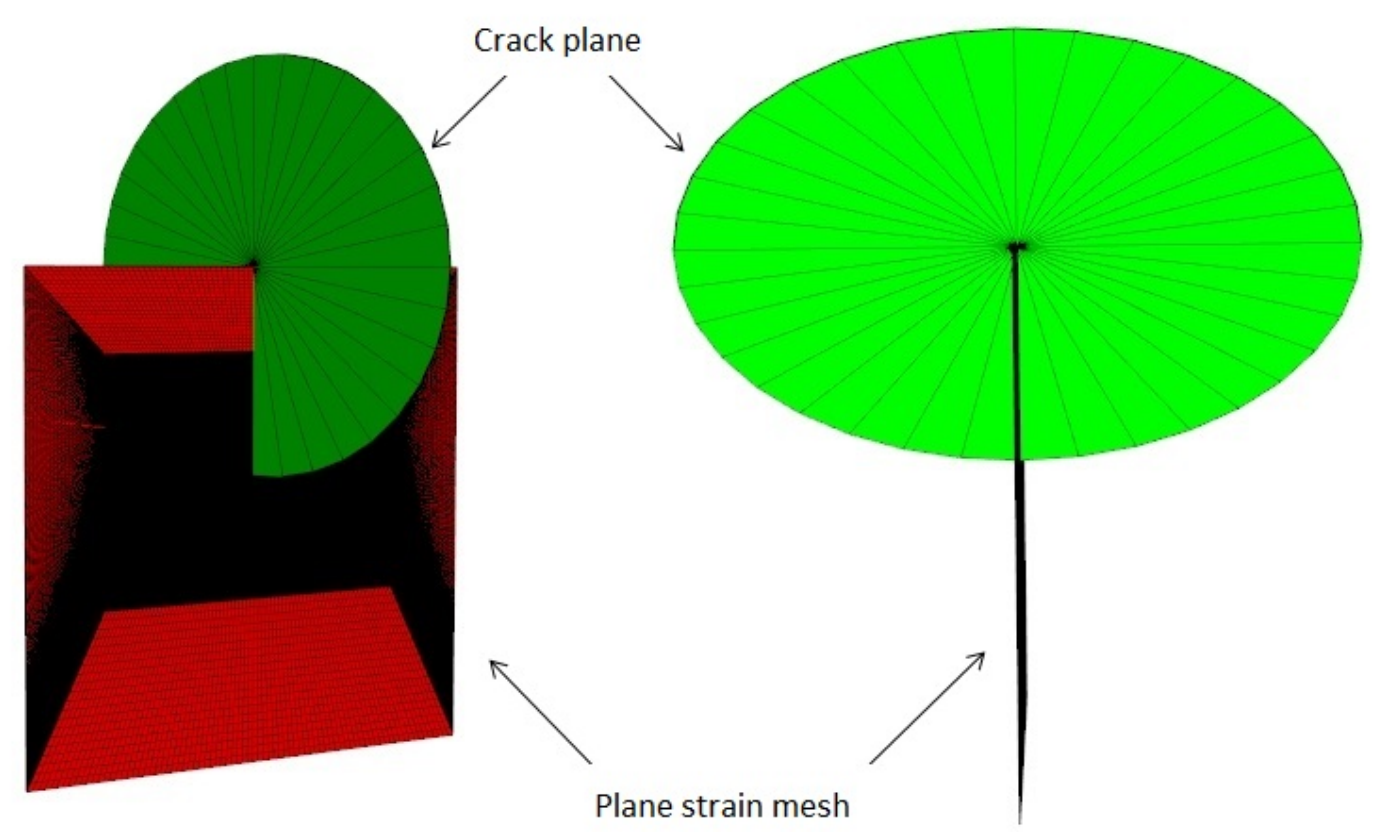

Figure 4.15: Example of an elliptical crack mesh that is perpendicular to the 2D plane strain mesh

- After setting the crack analysis parameters and running the project with a crack, the following result of the material force was acquired and depicted in Figure 4.16. The highest value of the material force was found to be $1.91 \times 10^{3}$. Using equations 4.5 and 4.6 the values of the J-integral and SIF are derived. The stress intensity factor for a crack length of $1 \mathrm{~mm}$ is $20.9 \mathrm{MPa} \sqrt{m}$. 


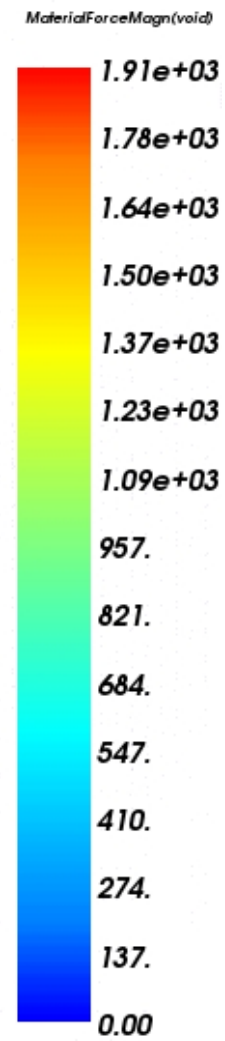

Figure 4.16: Material force vectors competed with VrSuite for a crack length of 0.5 $\mathrm{mm}$.

- In order to compute the number of cycles needed for crack to grow from 0.5 $\mathrm{mm}$ to $0.7 \mathrm{~mm}$, a crack is extended to a length of $0.7 \mathrm{~mm}$. The same analysis parameters and boundary conditions are used as in the analysis above. The result of the material force is shown on the Figure 4.17 with the highest value of $6.94 \times 10^{3}$. The SIF for this crack length is $40 \mathrm{MPa} \sqrt{m}$. 


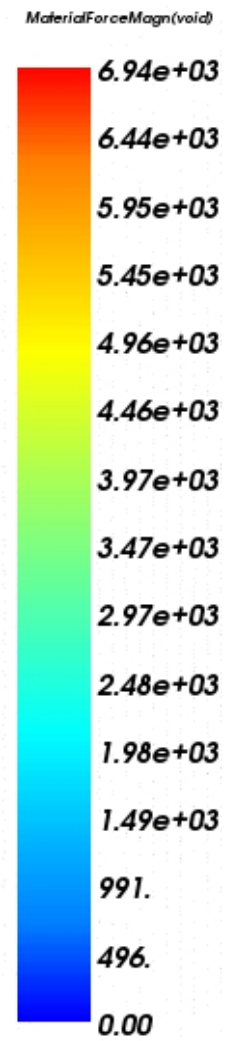

Figure 4.17: Material force vectors competed with VrSuite for a crack length of 0.7 $\mathrm{mm}$.

- Knowing values of the SIF for both cases give a possibility to determine the number of load cycles needed for fatigue crack growth from $0.5 \mathrm{~mm}$ to $0.7 \mathrm{~mm}$. Material constants for the analysis are $C=6.9 \times 10^{-12}(\mathrm{~m} / \mathrm{cycles})(\mathrm{MPa} \sqrt{\mathrm{m}})^{-m}$ and $m=3.0$. The Paris-Erdogan equation 4.8 was used to determine that 1,262 cycles are necessary to grow the crack to the prescribed length.

\subsection{Automation of the fatigue analysis process}

Automation of the fatigue analysis process of welded structures can play an important role for industries in terms of saving time and avoiding manual mistakes done by a 
user while setting an analysis. Automation of the algorithm above for fracture and fatigue analysis can also help a user to analyse more structures at the same time.

The following steps have been done in order to simplify and automate the process of fracture analysis for a user:

1. Instead of the user's manual picking of a Gauss point with a highest value of the principal stress for possible location of fatigue crack, a set of Gauss points is chosen automatically, based on the value of highest principal stress. A set of points can have 1, 2 or 10 possible locations of cracks.

2. A fine crack mesh with a center at the Gauss point is created automatically at each chosen point. The user can then set desired value for the radius of the crack.

3. A plain strain crack mesh that is perpendicular to the crack mesh is created automatically for each Gauss point. The user is prompted to set an angle for a plane strain mesh with respect to a crack mesh.

4. For each specified point on the crack tip curve, a plane strain analysis is done with result values of the stress, displacement and material force.

5. From the result of material force at each point on the crack tip, the stress intensity factor is calculated automatically.

6. If the result of the material force vector is not clear, the value of the SIF is computed automatically from the CTOA.

7. In terms of fracture analysis, the stress intensity factor is compared to the fracture toughness of the material to predict the risk of fracture.

8. The obtained results of stress intensity factor increment would allow the ParisErdogan equation to be solved automatically to determine the number of cycles 
for the crack to grow within a specified increment of crack length.

\subsubsection{Description of the wizard for an automated analysis}

In order to perform automated fracture and fatigue analysis, an automated crack wizard was implemented in VrWeld and will be described in the following section.

The wizard consists of four steps, each of which has a specified set of parameters that must be assigned values in order to achieve accurate results.

The first step is to input the parameters required to build an elliptical crack and plane strain crack mesh. During this step, the user sets the path to the 3D project with results of the computed transient temperature, microstructure, stress, strain and displacement.

The following parameters are required to create a crack:

- "crackLength" - desired length of the crack in $m$. (e.g. 0.006)

- "partWidth" - width of the crack plane strain mesh in $m$. (e.g. 0.008)

- "partHeight" - height of the crack plane strain mesh in $m$. (e.g. 0.01)

- "coreWidth" - width of the core region with refined mesh needed for a crack tip, inside the crack plain strain mesh in $m$. (e.g. 0.0005)

- "coreHeight" - height of the core region with refined mesh needed for a crack tip, inside the crack plain strain mesh in $m$. (e.g. 0.004)

- "nbElementsInCoreW" - number of elements along the width in core region with refined mesh. (e.g. 50)

- "nbElementsInCoreH" - number of elements along the height in core region with refined mesh. (e.g. 600) 
- "nbElementsInRadius" - number of elements in radius around the core region up to the boundary of the crack plain strain mesh. (e.g. 20)

- "nbElementsPenetration" - number of elements in the core that crack will penetrate. (e.g. 300)

- "debugFlag" - helps to check if the crack plane mesh can be created with the parameters set above. It can be set to 0 or 1 .

- "ab" - defines dimension of the elliptical crack in $m . a$ is equal to half of the length of an elliptical crack and $b$ is equal to half of the width of an elliptical crack. (e.g. 0.008 0.006)

- "nbLocations" - defines the number of the cracks that will be introduced to the specimen. (e.g. 1)

- "angles" - defines the angle between an elliptical crack and crack plane in radians. (e.g. 1.5708)

After all of the parameters listed above are specified in a GUI, the auto crack mesh can be created by clicking the "Make AutoCrack meshes" button. The first step of the wizard is displayed in the Figure 4.18. 


\section{Step $1 / 4$}

\section{Setup crack 2D mesh parameters}

\begin{tabular}{|c|c|}
\hline Structura I Lad 3D Project: & Project \\
\hline tlmeStep: & -1 \\
\hline partkd: & 1201 \\
\hline crackLength: & 0.006 \\
\hline partwidth: & 0.008 \\
\hline partHekght: & 0.01 \\
\hline coreWhdth: & 0.0005 \\
\hline coreHekght: & 0.004 \\
\hline nbElementsinCorew: & 50 \\
\hline nbElementsincoreH: & 600 \\
\hline nbElementsinRadlus: & 20 \\
\hline nbElementsPenetration: & 300 \\
\hline debugFlag: & 0 \\
\hline ab: & 0.0080 .006 \\
\hline nbLocatkns: & 1 \\
\hline angles: & 1.5708 \\
\hline
\end{tabular}

\section{Make AutoCrack meshes Vlew AutoCrack mesh}

Figure 4.18: First step of an automated crack wizard

The second step allows user to visualize the freshly created elliptical crack and plane strain crack mesh (with or without the 3D specimen), so the user can make sure that all of the parameters were set correctly. Selection of correct indices is used to aid in visualization of the required crack among the all of the cracks that have been made.

The following parameters are required for this step:

- "Crack3D Location Indices" - defines which particular plane strain crack mesh user wants to visualize among all that have been created. (e.g. 1)

- "Crack3D Angle Indices" - defines at which angle user wants to visualize the plane strain crack mesh relatively to an ellipse crack. (e.g. 1) 
- "Ellipse Location Indices" - defines which particular elliptical crack mesh user wants to visualize among all that have been created. (e.g. 1)

- "ViewCrack2D" button - visualizes the plane strain crack mesh in twodimensional space.

- "ViewCrack3D" button - visualizes the plane strain crack mesh in threedimensional space relatively to the 3D specimen.

- "ViewEllipse" button - visualizes an elliptical crack mesh.

- "ViewAll3D" - visualizes an elliptical crack as well as the crack plane strain mesh on the $3 \mathrm{D}$ object.

Example of the second step is shown on the Figure 4.19.

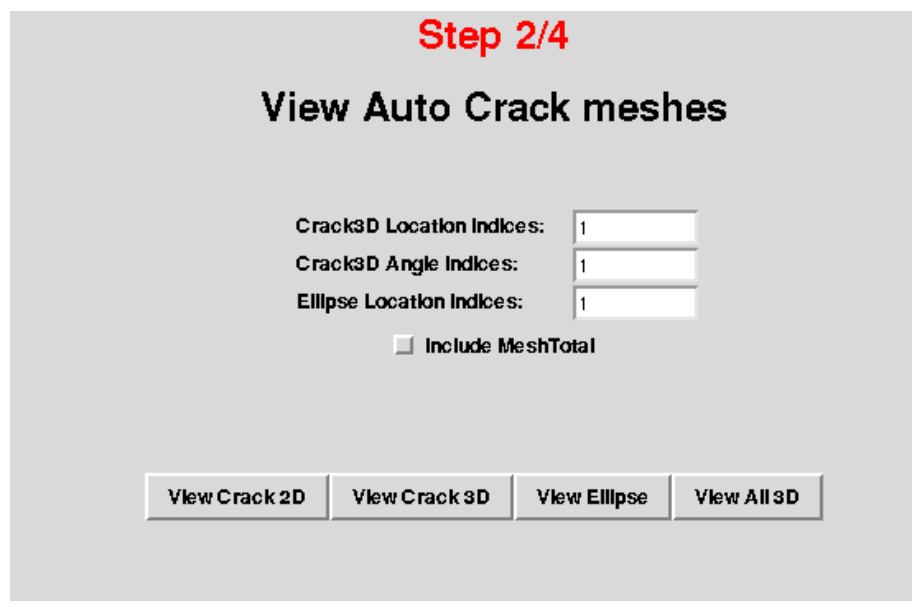

Figure 4.19: Second step of an automated crack wizard

The third step defines the solver parameters used for an analysis.

The following parameters are required for the third step:

- "nbNRIterations" - defines the number of Newton-Raphson iterations for an analysis. (e.g. 100) 
- "convergenceCriteriaNR" - defines the convergence criteria for an analysis. (e.g. $\left.1 \mathrm{e}^{-05}\right)$

- "materialForceFlag" - solves the material force. (e.g. 1)

- "Solve Location Indices" - defines which particular crack location will be analysed. (e.g. 1)

- "Solve Angle Indices" - defines which angle will be used for an analysis. (e.g. 1)

After all solver stress parameters are defined, the stress analysis can be run. The third step of the wizard can be seen on the Figure 4.20.

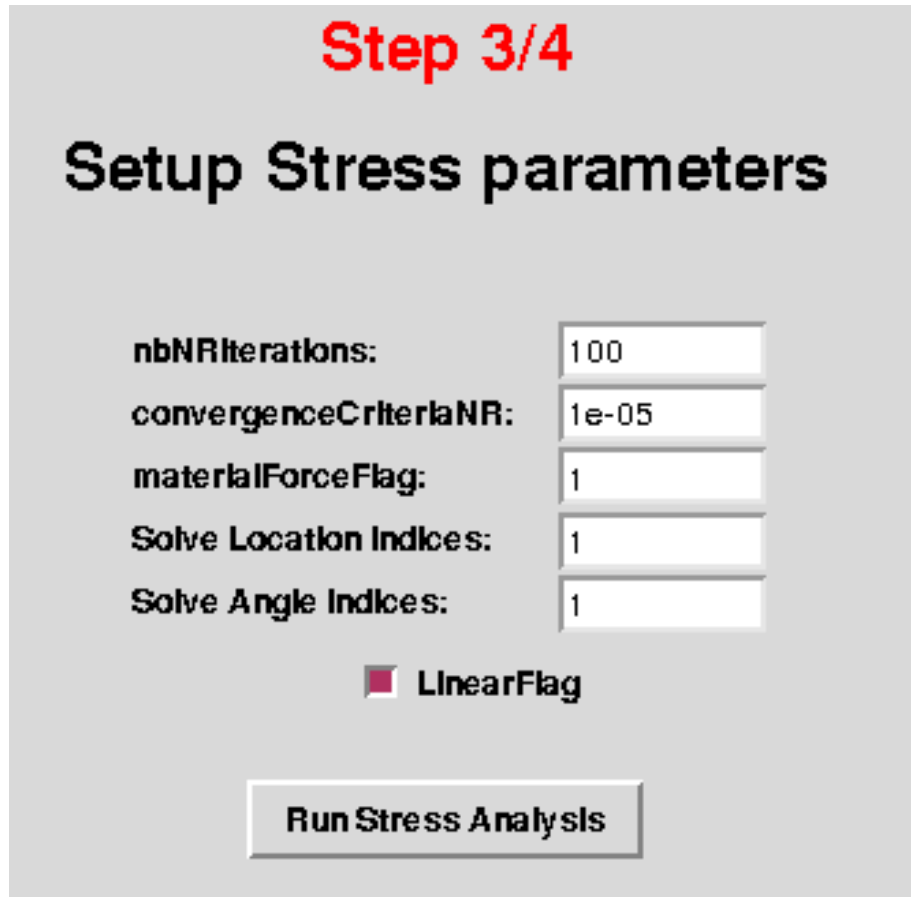

Figure 4.20: Third step of an automated crack wizard

After the analysis has been run and the results have been obtained, the fourth step is used to help develop the required report. During this step a user can set the formatting parameters for the report. Last step is displayed on the Figure 4.21. 


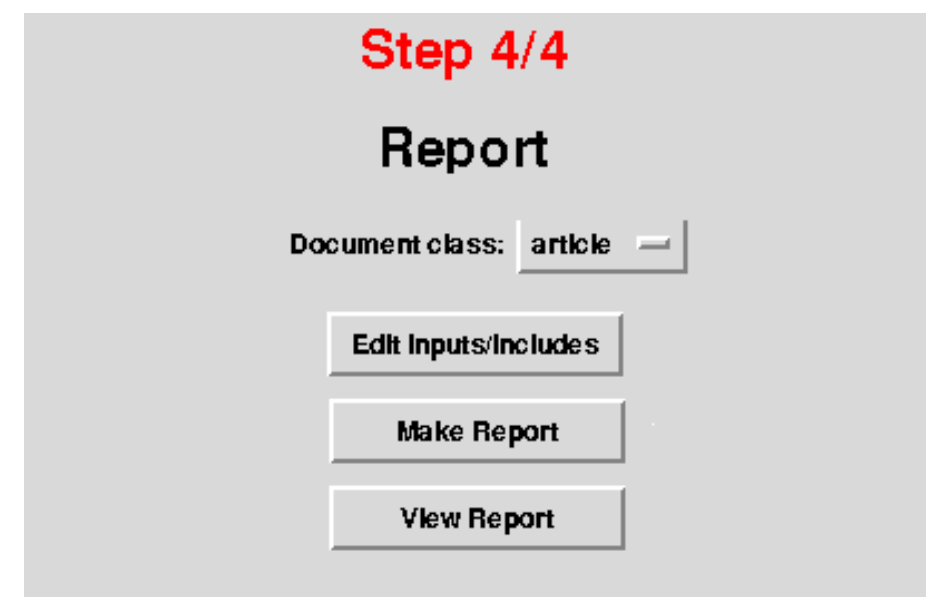

Figure 4.21: Fourth step of an automated crack wizard

\subsection{Automated analysis of the CTOA of the crack faces}

The following analysis investigates the CTOA field of the crack faces solved once for materials with an elastic behaviour and once for an elastic-plastic behaviour.

The Crack Tip Opening Angle criterion determines the fracture behavior of a stable crack extension [48]. Specimens that are used for the CTOA test usually stretch the tip of the crack prior to a failure because of some plastic deformation at the crack tip [49]. Figure 4.22 shows the difference between an analysis with an elastic material that has a sharp crack tip an analysis with an elastic-plastic material that has blunting crack tip because of the plastic deformation. 


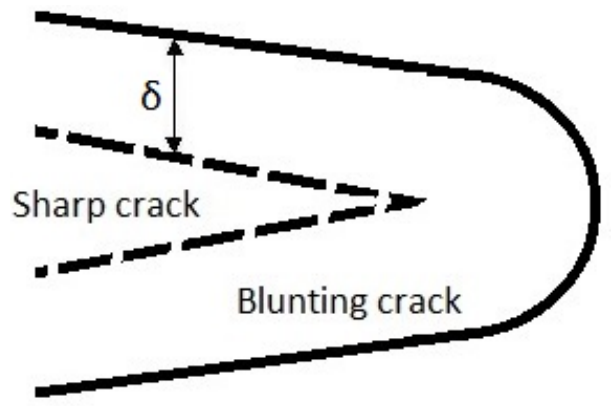

Figure 4.22: Displacement at the crack tip of an analysis with an elastic material sharp crack and an analysis with an elastic-plastic material - blunting crack.

In order to perform this study, a 3D parallelepiped was created with the top and bottom faces subjected to a vertical cyclic load. It has dimensions of $20 \mathrm{~mm} \times 50 \mathrm{~mm}$ $\times 20 \mathrm{~mm}$ and is displayed on the Figure 4.23. The material used is BABAnderson steel. The mesh used for 3D analysis is 8 node brick elements. The load consists of one cycle and is applied linearly with $20 \mathrm{MPa}$ increment in each time step until it reaches the peak value of $100 \mathrm{MPa}$. After the peak value of the load is reached, the load steps monotonically decrease to the initial condition. To make sure that the load was applied correctly, the plot with the result of the stress vs time step and the plot with the result of the displacement vs time step were built and represented in the Figure 4.24. 


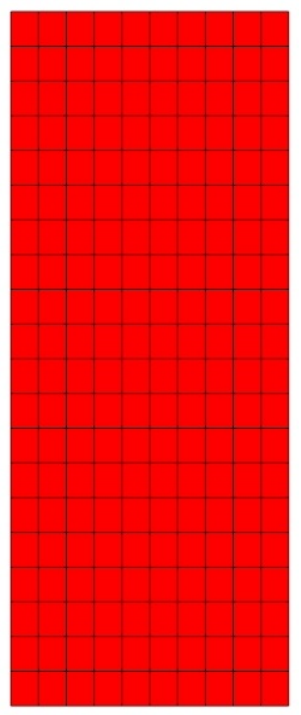

Figure 4.23: Geometry of the 3D object.

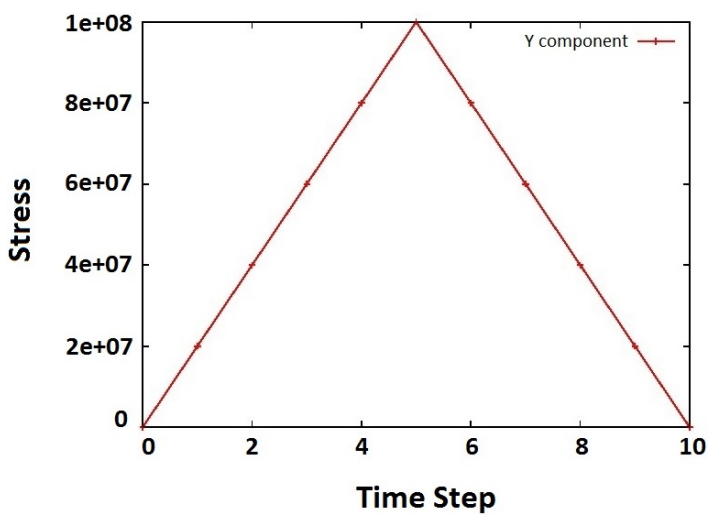

a)

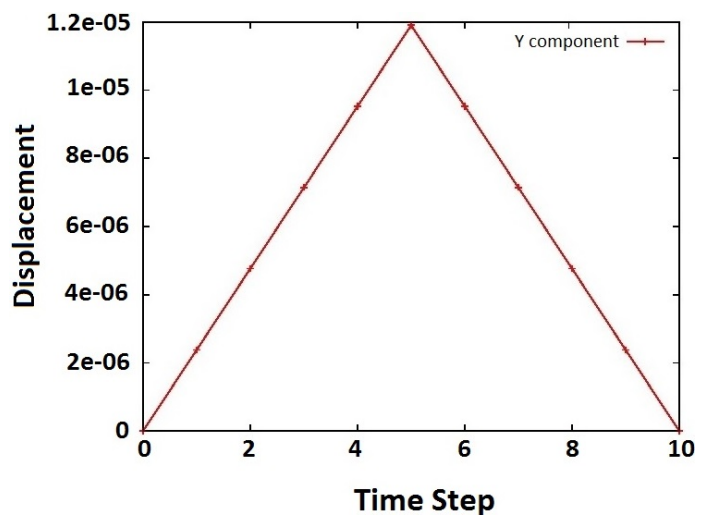

b)

Figure 4.24: a) Traction BC applied to the 3D object vs time step b) Displacement vs time step for the $3 \mathrm{D}$ object.

As soon as the 3D stress analysis is done, an automated 2D crack analysis can be performed. The location of the crack in the 3D specimen is set to be chosen automatically at the fifth time step of the 3D stress analysis which corresponds to the maximum value of the applied stress. The length of the crack is set to $2 \mathrm{~mm}$. 
To evaluate the crack tip blunting radius, two separate crack analyses were done. One of the analysis prescribed the material to an elastic behaviour and another analysis prescribed the material to an elastic-plastic behaviour. When both analyses are conducted and the results of the displacement are obtained, a plot of the displacement of the crack face in the direction perpendicular to the crack plane vs distance from the crack tip in the direction of the crack initiation is made for each solve and time step. After plots of the displacement for an elastic and an elastic-plastic behaviour are done, they are combined into one plot for each time step. An example of such plot for time step five can be found on the Figure 4.25.

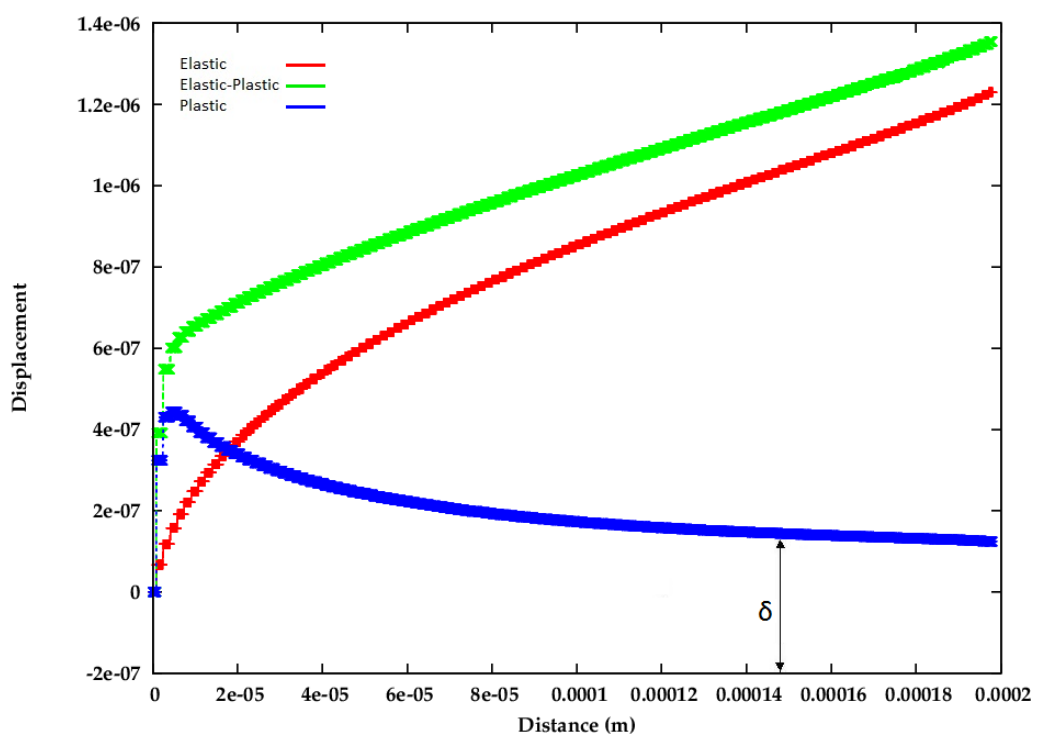

Figure 4.25: Displacement vs Distance from the crack tip for the time step five in Figure 4.24. See Figure 4.22 for a schematic of elastic and the elastic-plastic displacement for given applied traction BC.

It can be noticed from the plot that displacement obtained with an elastic material behaviour obeys the principles of the linear elastic fracture mechanics and represents the relation to the square root of the distance from the crack tip. The displacement obtained with an elastic-plastic behaviour of the material is above the displacement obtained with an elastic solve. The result of the plastic material displacement was 
derived as the difference between an elastic-plastic displacement and an elastic displacement [50]. It represents the plastic zone at the crack tip. As it can be noticed from the result of the plastic displacement there is some plastic deformation at the tip of the crack which cause the stretching of the crack tip as shown in the Figure 4.22. At some distance from the crack tip, the result of the plastic displacement becomes a constant, because of the end of the plastic deformation at the tip of the crack, and makes it possible to measure the amount of plastic blunting at the crack tip shown in the Figure 4.25. The difference in displacement between an elastic-plastic material behaviour and an elastic material behaviour that is represented by $\delta$ on the Figure 4.25 equals to the difference in displacement $\delta$ on the Figure 4.22.

Once the constant values of the crack tip blunting for each time step are extracted, the plot of the crack tip blunting evolution vs stress applied to the 3D specimen for each load step is created and shown in Figure 4.26. This plot indicates that the crack tip blunting increases with each load step until it reaches the maximum load, then it gradually decreases. The crack tip blunting does not return to the initial condition due to the effect of the crack tip plastic zone. The point on the horizontal axis where the stress returns to zero indicates the crack tip radius caused by the plastic displacement at zero load. 


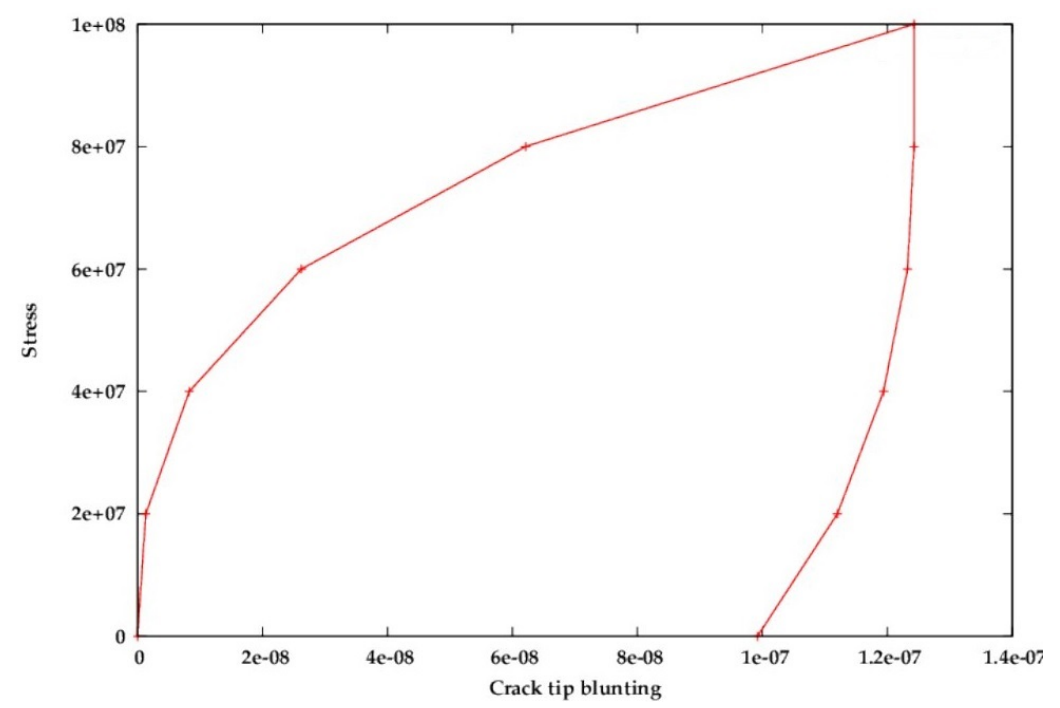

Figure 4.26: The result of the crack tip blunting evolution is plotted vs the applied stress for the load steps shown in Figure 4.24.

\subsection{Automated fatigue analysis of the WIC test and girth weld}

\subsubsection{Introduction and geometry of the specimens}

The goal of the WIC test is to measure the risk of hydrogen cracking in a weld. The risk of hydrogen cracking is strongly correlated with the residual tensile stress, the hardness of the metal, the presence of martensite and the presence of hydrogen. The design of the WIC test specimen is comprised of two strips of metal, possibly taken from a pipe, that are to be welded together. The strips are placed on a backing plate with a shim and stiffener. Geometry of the WIC specimen can be seen in Figure 4.27(a). The material used is Gr. 483 steel. The 3D FEM mesh consists of 8 node brick elements. The weld joint consists of two plates welded together by a root pass, with the cross-section displayed in Figure 4.27(b). The size of each plate is $150 \mathrm{~mm} \times$ $12.7 \mathrm{~mm} \times 50 \mathrm{~mm}$. The bevel angle between the plates is $60^{\circ}$. 
Since the WIC test has a static nature and the purpose of the test doesn't envisage the use while cycling loading, the objective of this study will include automated analysis of the WIC test in terms of the fracture mechanics.

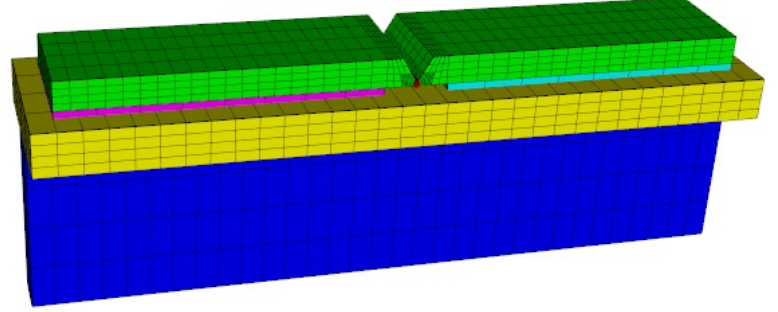

a)

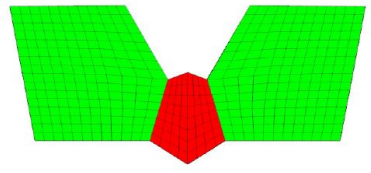

b)

Figure 4.27: a) Geometry of the WIC specimen with turned on mesh b) Cross-section of the weld joint of the WIC specimen.

The most common type of failure for pipe welds is corrosion fatigue. It forms due to the contact of the pipe, subjected to a tensile load, with corrosive environment surrounding the pipe. The diameter of the pipe for the current analysis is $0.9378 \mathrm{~m}$, the wall thickness is $0.0127 \mathrm{~m}$ and the length of each side of the welded pipe is equal to $0.9 \mathrm{~m}$. The material used is Gr. 483 steel. The 3D FEM mesh consists of 8 node brick elements. The geometry of the girth weld is represented on the Figure 4.28 a) and the weld joint cross-section can be seen on the Figure $4.28 \mathrm{~b}$ ). The objective of this case will include application of the automated fatigue analysis of the girth weld. 


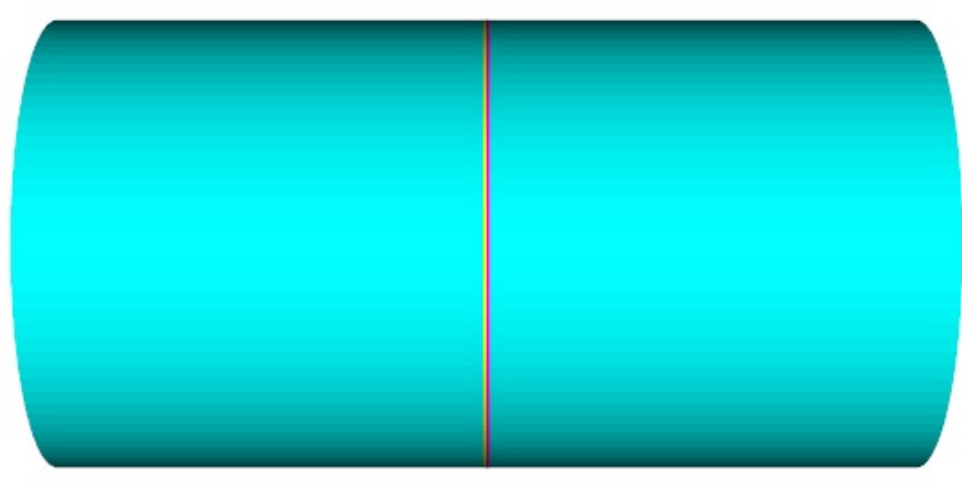

a)

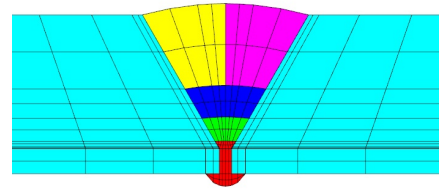

b)

Figure 4.28: a) a) Geometry of the pipe girth weld b) cross-section of the weld joint of the pipe girth weld.

\subsubsection{Setup and analysis of the test cases}

The following section describes the setup parameters that have been used in order to automatically assess the WIC test specimen and girth weld in terms of the fracture and fatigue analysis respectively.

Once the mesh for the WIC test is built and the root pass is welded, a two dimensional crack analysis can be done. A 2D crack analysis uses results of the traction and displacement from the 3D project where welding of the WIC test specimen was done. In order to determine the most likely crack initiation sites the automatic crack analysis was configure to identify the Gauss point with the highest principal stress in the root of the weld. At this position an elliptical crack was placed with the $2 \mathrm{D}$ crack plane perpendicular to the crack as shown in Figure 4.29 a) and b). The size of the crack for this case was set to $1 \mathrm{~mm}$. The stress analysis of the crack was performed using linear elasticity. After stress analysis is done, the report with results is generated automatically. The report includes the following results: value of the SIF, visualization of the displacement field, visualization of the stress field, visualization of the material 
force vectors and plot of the stress from the crack tip to the bottom of the $2 \mathrm{D}$ crack plane. For this case, the value of the SIF was calculated from the displacement at the crack tip, as described in the Section 2.9 by the use of the Equation 2.11. An example of the report is given in Appendix A.

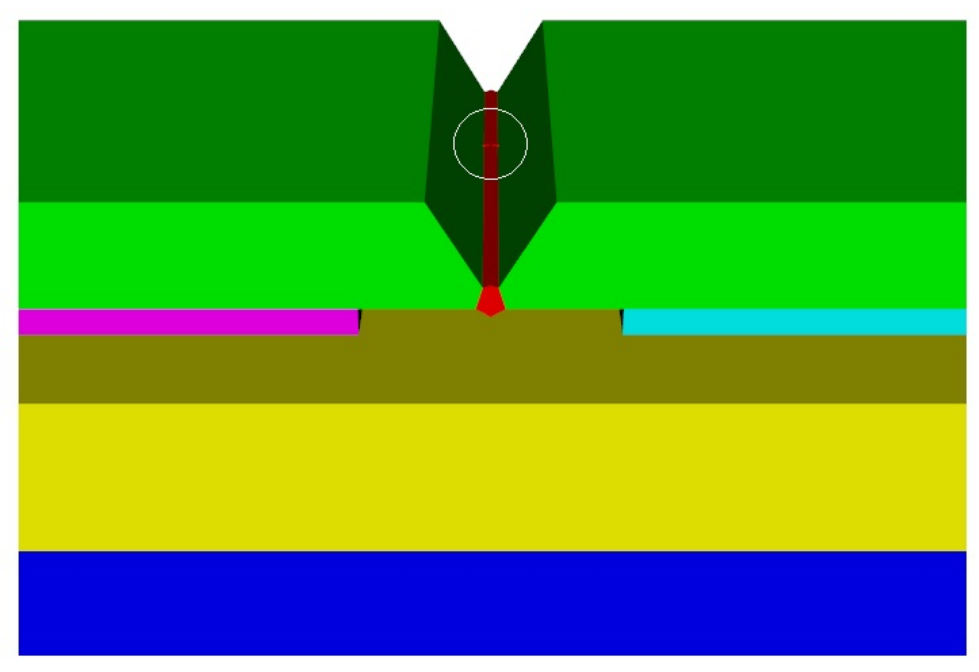

a)

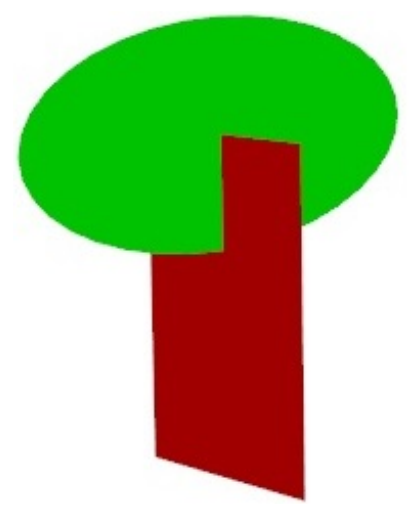

b)

Figure 4.29: a) Location of the crack in the WIC test specimen b) An elliptical crack mesh with perpendicular to it $2 \mathrm{D}$ crack plane in the WIC test specimen with turned-off parts.

As it can be seen from the results obtained from the $2 \mathrm{D}$ crack analysis, the value of the stress intensity factor derived from material force vector for the crack length of $1 \mathrm{~mm}$ equals to $45.8 \mathrm{MPa} \sqrt{m}$. Fracture toughness $K_{I C}$ values for steel materials have a range between 50 to $200 \mathrm{MPa} \sqrt{m}$ [51]. This means that if the crack of $1 \mathrm{~mm}$ length appears in the root pass of the WIC test geometry it will not cause a rupture if the fracture toughness of the structure is assumed to be $50 \mathrm{MPa} \sqrt{m}$, but the failure might be imminent.

The pipe weld was subjected to a static internal pressure of $10 \mathrm{MPa}$ by applying 
pressure to the internal faces of the pipe. A $2 \mathrm{D}$ crack analysis uses results of the traction and displacement from the 3D project where welding and subsequent loading of the pipe weld is performed. The position of the surface crack that has been picked automatically by the wizard based on the results of the principal tensile stress is shown in the Figure 4.30 a) with a close look to the elliptical crack and $2 \mathrm{D}$ crack plane in the Figure $4.30 \mathrm{~b})$.

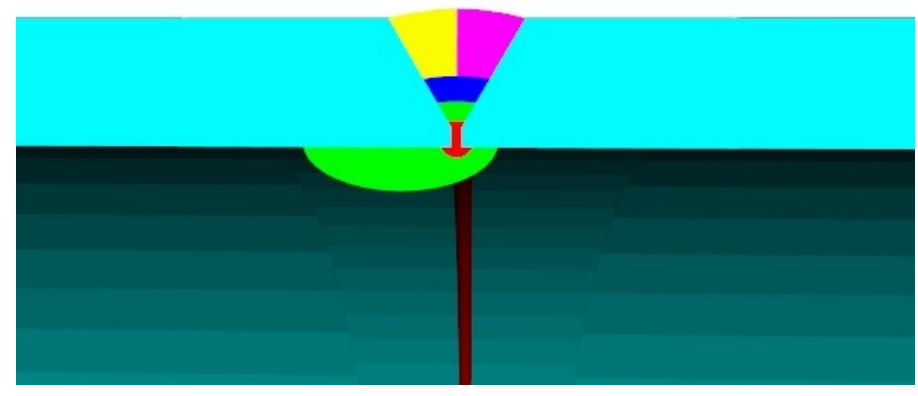

a)

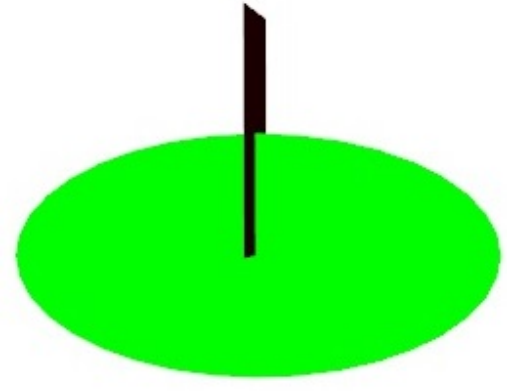

b)

Figure 4.30: a) Location of the crack in the pipe weld b) an elliptical crack mesh shown in light green with the plane strain 2D mesh perpendicular to the crack tip in the pipe girth weld. The pipe parts are made invisible to show the crack and the plane strain $2 \mathrm{D}$ mesh.

To grow the length of the crack in the pipe weld from $4 \mathrm{~mm}$ to $6 \mathrm{~mm}$, a two $2 \mathrm{D}$ stress analyses were conducted. Both of the analyses were performed using linear elasticity. Once the analyses are completed and the results are obtained, the autoreport is generated. Copies are shown in Appendix B, C. Stress intensity factor for the crack length of $4 \mathrm{~mm}$ is $66.9 \mathrm{MPa} \sqrt{m}$. Stress intensity factor for the crack length of $6 \mathrm{~mm}$ is $135.9 \mathrm{MPa} \sqrt{m}$. When the SIF value for both crack lengths is obtained, the determination of the numbers of cycles to grow a crack can be performed by utilization of the Paris-Erdogan equation 4.8. The number of cycles to grow a crack from $4 \mathrm{~mm}$ to $6 \mathrm{~mm}$ is 355 cycles. 


\section{Chapter 5}

\section{Conclusion and possible future works}

The current work reviews several studies conducted on fatigue analysis in Section 1.2. By analysing works performed by other researches it was feasible to identify possible extension of scope in fatigue analysis, as well as to define new areas of study.

The assessment of residual life of the welded structures in this thesis is done through development of an algorithm for performing fracture mechanics analysis and fatigue analysis using the VrSuite software. The objectives of this investigation included:

- Define an algorithm to automate the determination of the linear elastic stress intensity factors in welding components containing residual stresses due to welding in combination due to in-service loads;

- Predict the crack rate of crack propagation in the welded structures due to in-service load cycles;

Initially, VrSuite software was verified by comparison to computed stress results with an analytical solution. For this purpose, in Sections 4.1.1.1, an analysis was conducted on a 2D plate with a hole in the center of it subjected to tensile load. The idea of this test was to compute the maximum stress in the plate and compare it to an established analytical solution. As a result, the comparison of results showed a good agreement with an error of less than 5\%. Later in this section the direction of 
the material force vectors was compared to the one obtained from the literature. As can be seen from the comparison in Section 4.1.1.2, the distribution of material force vectors computed by VrSuite is consistent with result of material force vector taken from the paper [46].

After verification of the stress results obtained by VrSuite an edge crack was introduced into the 3D plate with displacement applied to the top and bottom side of the plate as described in Section 4.1.2.2. The aim of the analysis was to compare the result of the stress intensity factor computed by FEM with the help of the material force and J-integral to the value derived by using the analytical equation taken from [20] for an edge crack, to verify the results obtained by VrSuite. As can be seen in Section 4.1.2.2, the stress intensity result obtained by FEA had an error of only $2.5 \%$ compared to an analytical solution which indicates a good agreement between the results. In section 4.1.2.4 a modified form of the Paris-Erdogan equation is implemented to compute the number of load cycles required to grow a crack to a specified size.

Section 4.2 describes the procedure for conducting a manual fatigue analysis for a laser weld. It includes a description of the geometry used for analysis and provides information on the setup and parameters used in this case as well as discussion of the obtained results.

A description of the automated fatigue analysis is given in Section 4.3. The section concentrates on the difference between manual and automated fatigue and fracture analysis of the welded components. An explanation of the role of the wizard and steps of the automated analysis is given.

The use of an automated fracture and fatigue analysis is presented for a WIC test specimen and a pipe girth weld. Section 4.5 includes information about the geometry of each specimen and parameters that were used for each case as well as provides the results obtained from the automated analysis. 


\subsection{Future direction of work}

Although the research described in this thesis has provided new insight into an automated fatigue and fracture analysis of welded components, there are opportunities for improvement. This section expands upon some potential enhancements that may be incorporated in future work:

- Extend the ability to analyze not only surface cracks but sub-surface cracks as well.

- Perform further analysis on additional specimens with varying geometries, including the cases with gusset joints.

- Perform analysis with cycling load patterns.

- Implement an algorithm for predicting fatigue crack growth based on Pommier's time-derivative crack propagation equations [50]. 


\section{References}

[1] Stout R.D., Wayman C.M. A study of factors affecting the strength and ductility of weld metal. Welding Journal, 5, May 1958.

[2] Harrison J.D. Lessons from service fatigue failures. The Welding Institute Research Bulletin, 12, March 1980.

[3] Rethwisch D. G., Callister W. D. Materials science and engineering: An introduction. Hoboken, N.J: John Wiley, 7 edition, 2007.

[4] Macdonald K. A. Fracture and Fatigue of Welded Joints ans Structures. Woodhouse Publishing Limited, Cambridge, 2011.

[5] B. Schwerdt D., Berger C. \& Pyttel. Very high cycle fatigue is there a fatigue limit? International Journal of Fatigue, 33(1):49-58, 2011.

[6] Kim G. R., Kim Y. N., Kim M. H., Kim S. G., Lee K. E. \& Kim S. M. A comparative study for the fatigue assessment of a ship structure by use of hot spot stress and structural stress approaches. Ocean Engineering, 36(14):1067-1072, 2009 .

[7] Tveiten B. W. \& Moan T. Determination of structural stress for fatigue assessment of welded aluminum ship details. Marine Structures, 13(3):189-212, 2000.

[8] Maddox S. J. Review of fatigue assessment procedures for welded aluminium structures. International Journal of Fatigue, 25(12):1359-1378, 2003.

[9] Poutiainen I. \& Marquis G. A fatigue assessment method based on weld stress. International Journal of Fatigue, 28(9):1037-1046, 2006.

[10] Miller B., Metallurgical engineer, Knovel (Firm) and Wulpi D. J. Understanding how components fail. Materials Park, Ohio: ASM International., 3 edition, 2013.

[11] Anderson T. L. Fracture Mechanics: Fundamentals and Applications. Taylor \& Francis Group., 3 edition, 2005. 
[12] Miannay D. P. Fracture mechanics. New York: Springer, 1998.

[13] McLean D. Mechanical properties of metals. 1962.

[14] Irwin G. R. Analysis of stresses and strains near the end of a crack traversing a plate. Journal of Applied Mechanics, 24:361-364, 1957.

[15] Erdogan E. Fracture mechanics. International Journal of Solids and Structures, $37: 171183,2000$.

[16] Willis J. R. and Harlin G. The inuence of crack size on the ductile-brittle transition. Proceedings of the Royal Society of London. Series A, Mathematical and Physical Sciences, 415(1848):197-226, 1988.

[17] Suresh S. Fatigue of materials. Cambridge: New York; Cambridge University Press, 2 edition, 1998.

[18] Paris P. C., Irwin G. R.and Tada H. The stress analysis of cracks handbook. 1973.

[19] Cartwright D. J. \& Rooke D. P. Compendium of stress intensity factors. London: H.M.S.O., 1976.

[20] Tuba I.S., Chan S.K. and Wilson W.K. On the finite element method in linear elastic fracture mechanics. Engineering Fracture Mechanics, 2:1-17, 1970.

[21] Cotterell B. Notes on the paths and stability of cracks. International Journal of Fracture Mechanics, 2(3), 1966.

[22] Cherepanov G. P. Cracks in solids. International Journal of Solids and Structures, 4(8):811-831, 1968.

[23] Pook L. P., Marsh K. J., Knovel (Firm) \& Frost N. E. Metal fatigue. 1999.

[24] Ciavarella M., Cornetti P., Carpinteri A., \& Pugno N. A generalized paris law for fatigue crack growth. Journal of the Mechanics and Physics of Solids, 54(7):1333-1349, 2006.

[25] Erdogan F. and Paris P. A critical analysis of crack propagation laws. J. Basic Eng. Trans. ASME, page 528534, 1963.

[26] Knovel (Firm), Barsom J. M. \& Rolfe S. T. Fracture and fatigue control in structures: Applications of fracture mechanics. West Conshohocken, PA: ASTM, 3 edition, 1999. 
[27] Pook L. P. Linear elastic fracture mechanics for engineers; theory and applications. Portland: Ringgold Inc., 2000.

[28] Bower A. F. Applied mechanics of solids. Boca Raton: Taylor \& Francis, 2010.

[29] Griffith A. A. The phenomena of rupture and flow in solid. Philosophical Transactions of the Royal Society of London, 221: 163198, 1921.

[30] Gurses E. Aspects of energy minimization in solid mechanics: Evolution of inelastic microstructures and crack propagation. Master's thesis, University of Stuttgart, 2007.

[31] Qi Chen. Stress intensity factors and weight functions for cracks in fastener holes. Master's thesis, Carleton University, 2002.

[32] Min-Kang Seo and Soo-Jin Park. Interface Science and Composites. Academic Press, 2011.

[33] Eshelby J. The elastic energy-momentum tensor. Journal of Elasticity, 5:325-335, 1975.

[34] Coulombe M. Computational fatigue analysis of welded structures. Master's thesis, Carleton University.

[35] Barth F. J., Denzer R. and Steinmann P. Studies in elastic fracture mechanics based on the material forces method. International Journal for Numerical Methods in Engineering, 58:18171835, 2002.

[36] Steinmann P. and Kirchner N. On the material setting of gradient hyperelasticity. Mathematics and Mechanics of Solids, 12:559-580, 2007.

[37] Eshelby J.D. The force on an elastic singularity. Philosophical Transactions of the Royal Society of London, 244:87112, 1951.

[38] Barsoum R.S. On the use of isoparametic finite elements in linear fracture mechanics. Int. J. Num. Meth. Eng., 10:2537, 1976.

[39] Fricke W. Fatigue analysis of welded joints: State of development. Marine Structures, 16(3):185-200, 2003.

[40] Dr.Ali Sadiq Yasir. Study the effect of welding joint location on the fatigue strength and fatigue life for steel weldment. Asian Transactions on Engineering (ATE ISSN: 2221 - 4267), 2(4), 2012. 
[41] PD5500. Specification for unfired fusion welded pressure vessels. BSI, 2009.

[42] Aygul M. Fatigue analysis of welded structures using the finite element method. Master's thesis, Chalmers University of Technology, 2012.

[43] Dassault Systemes. Stress-life cycle curve, accessed May 22, 2016.

[44] Glinka G. Fatigue life prediction from the gy2 shell finite element stress data. 2006.

[45] Young W. C. and Budynas R. G. Roarks Formulas for Stress and Strain. 7 edition, 2002.

[46] Mueller R., Gross D. and Maugin G. Use of material forces in adaptive nite element methods. Computational Mechanics, 33:421434, 2004.

[47] Givoli D. and Zusman R. An adaptive finite element framework for fatigue crack propagation. International Journal for Numerical Methods in Engineering, 54:111135, 2002.

[48] Zhu X. and Joyce J. Review of fracture toughness (g, k, j, ctod, ctoa) testing and standardization. Engineering Fracture Mechanics, 85:1-46, 2012.

[49] Westmoreland Mechanical Testing and Research. Ctod test, Accessed November $13,2016$.

[50] Pommier S. and Risbet M. Time-derivative equations for fatigue crack growth in metals. International Journal of Fracture, 131(1):79-106, 2005.

[51] Kanninen M. F. and PopelarC. H. Advanced fracture mechanics. Oxford Engineering Science Series, 15:111135, 1985. 


\section{Appendix A}

\section{Auto-report of the fracture analysis of the WIC test}

Fracture analysis for the current project is tailored with the help of an automated crack wizard. Results obtained in the analysis are covered in this report.

The stress intensity factor can be computed using the equation below:

$$
K_{I}=\frac{2 S}{k+1} \sqrt{\frac{\pi}{2 l}}\left[4\left(u_{b 2}-u_{b 1}\right)-\left(u_{c 2}-u_{c 1}\right)\right]=45.8 M P a \sqrt{m}
$$

where: $K_{I}$ is the stress intensity factor for mode I, $S=8.08 \cdot 10^{10}$ is the shear modulus, $l$ is the length of the element in the crack tip region, $u_{b 2}$ and $u_{b 1}$ are top displacement and bottom displacement values of the crack faces taken at the middle of an element, $u_{c 2}$ and $u_{c 1}$ are top displacement and bottom displacement values of the crack faces taken at the corner of an element, $k$ is defined as:

$$
\begin{gathered}
k=(3-4 \nu)(\text { Plane srain }) \\
k=\frac{3-\nu}{1+\nu} \text { (Plane stress) }
\end{gathered}
$$

where: $\nu=0.3$ is the Poison's ratio. 
Now the result of the SIF can be compared to a known fracture toughness value. 

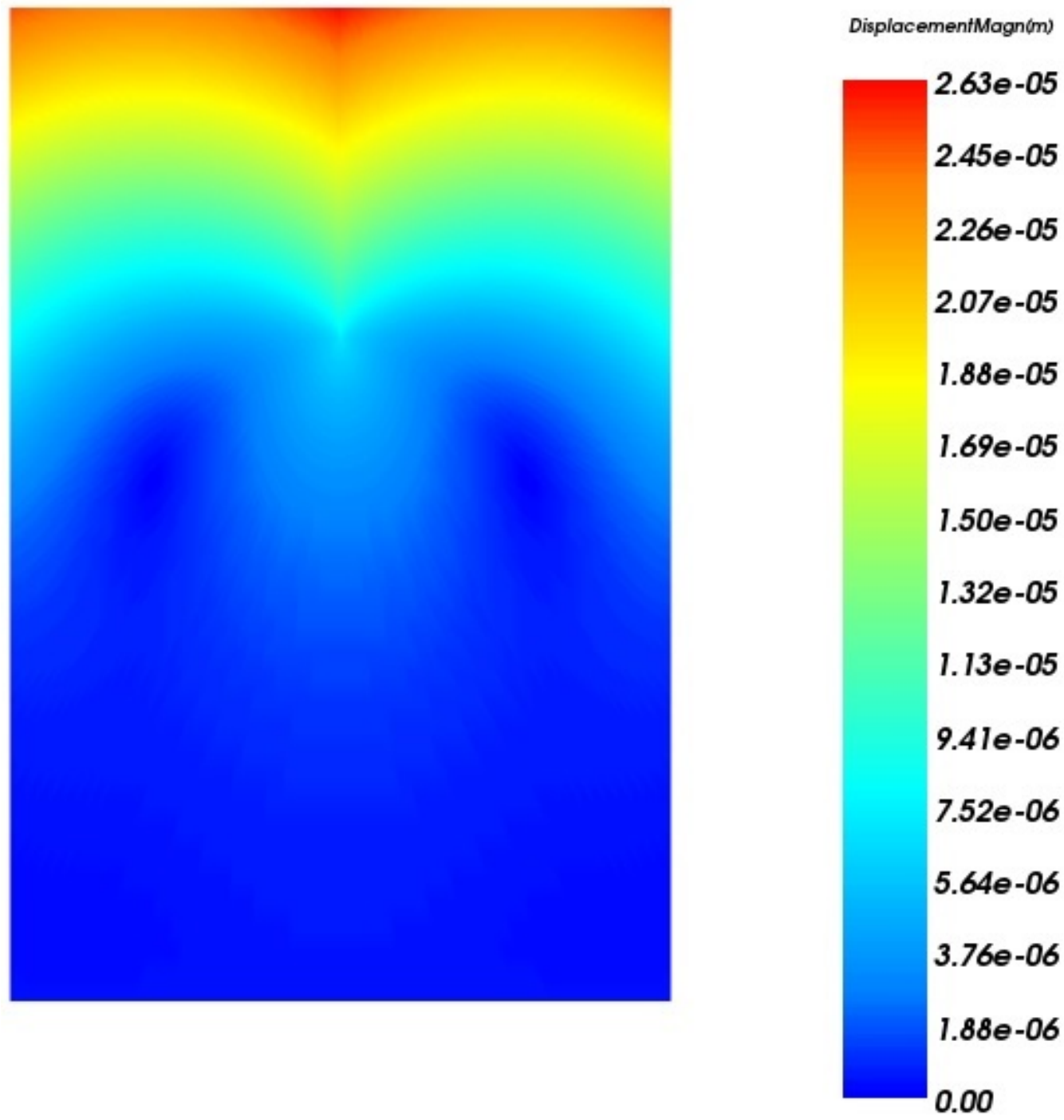

Figure A.1: The displacement field in a 2D plane strain mesh with a crack is shown. 


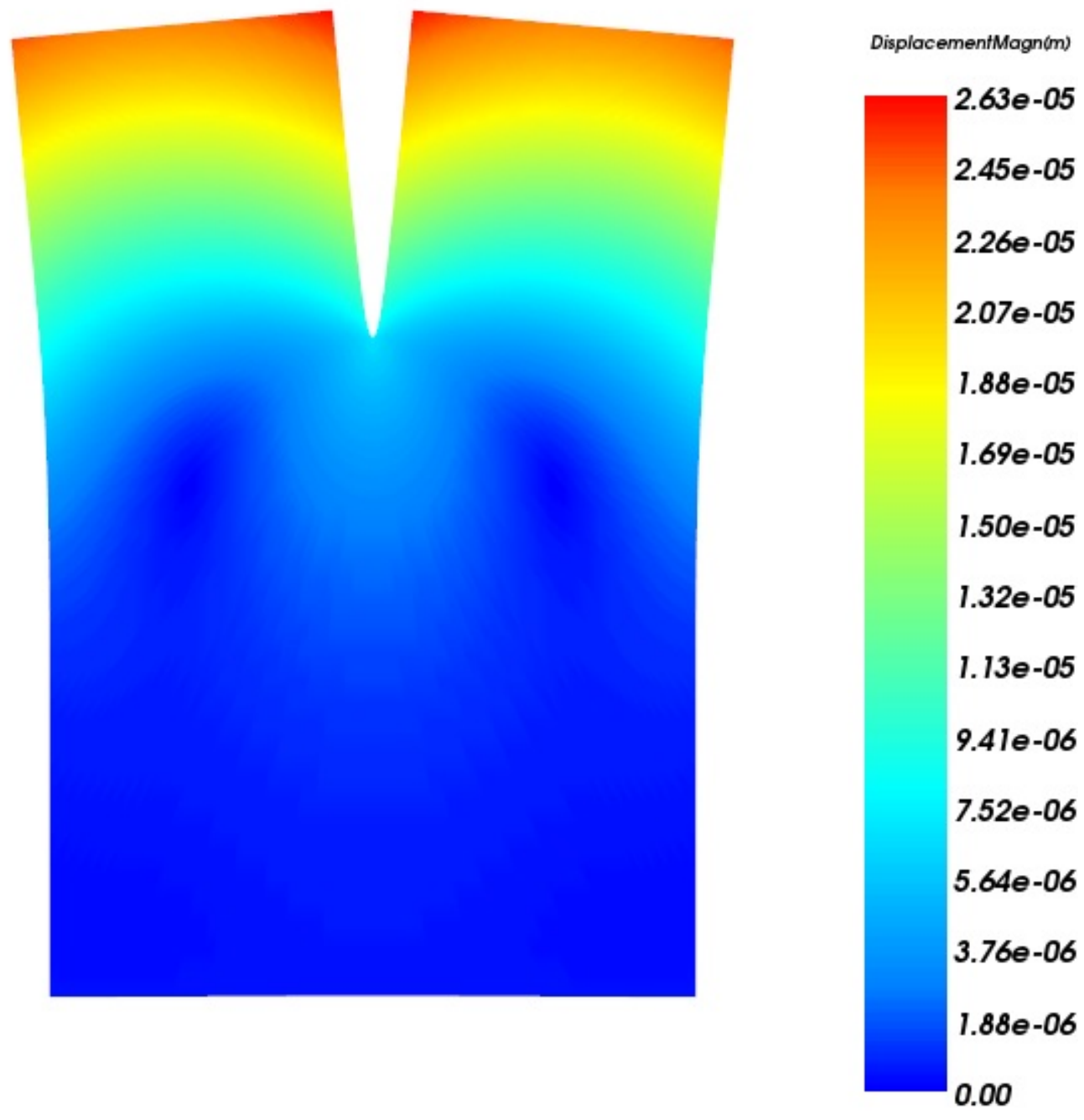

Figure A.2: The displacement field in a 2D plane strain mesh with a crack is shown. Magnified 5 times. 


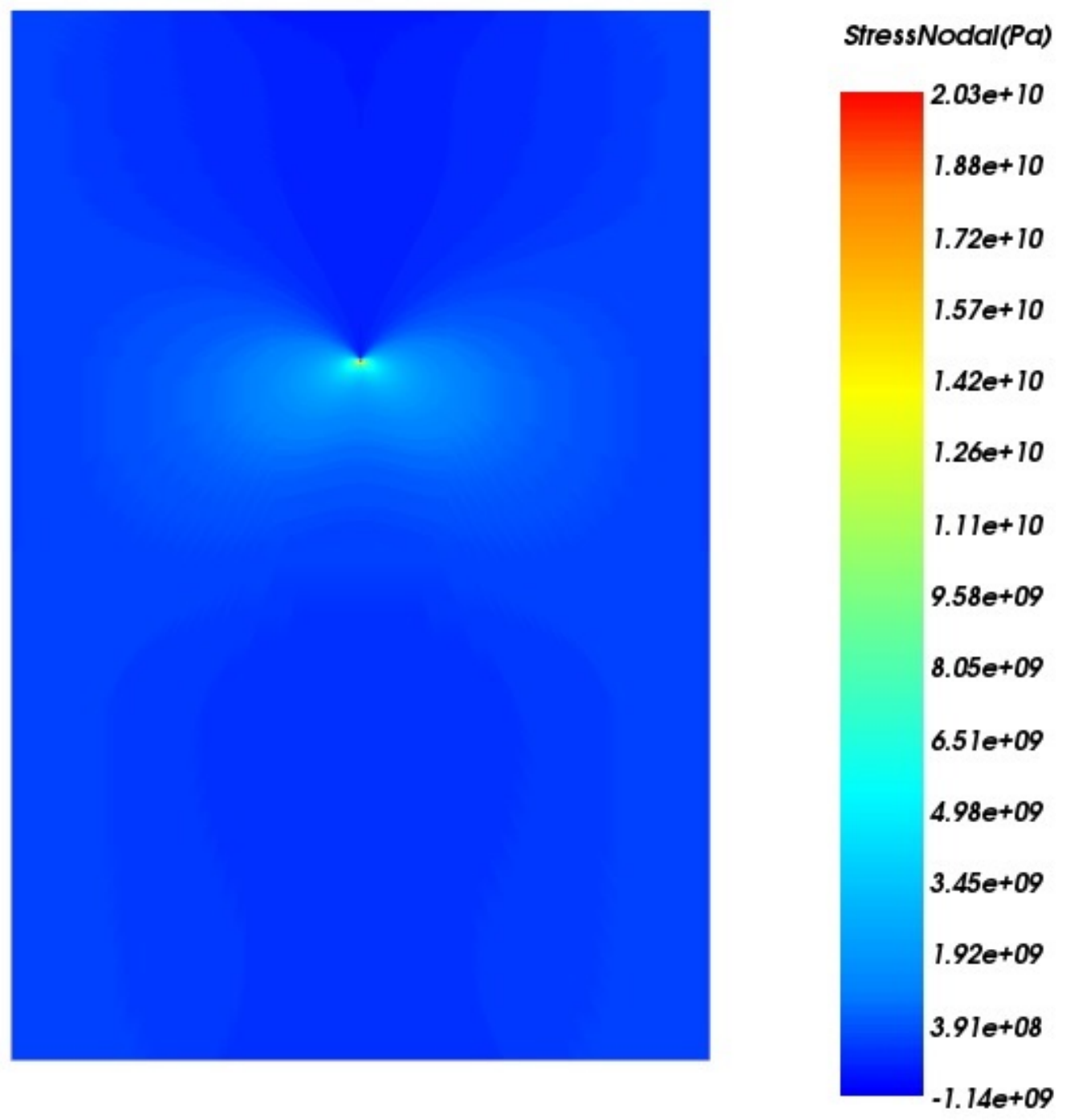

Figure A.3: The xx stress is shown in a 2D plane strain mesh with a crack. 


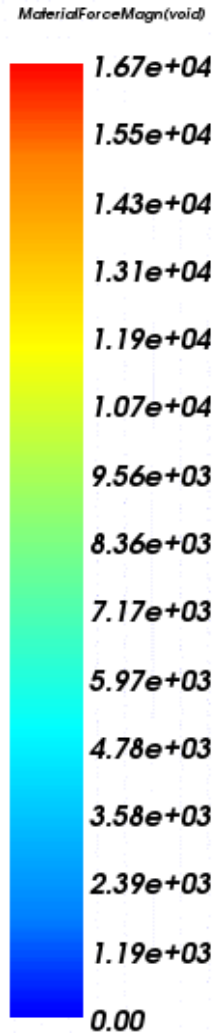

Figure A.4: The computed material force vectors near the crack tip in a 2D plane strain mesh. 


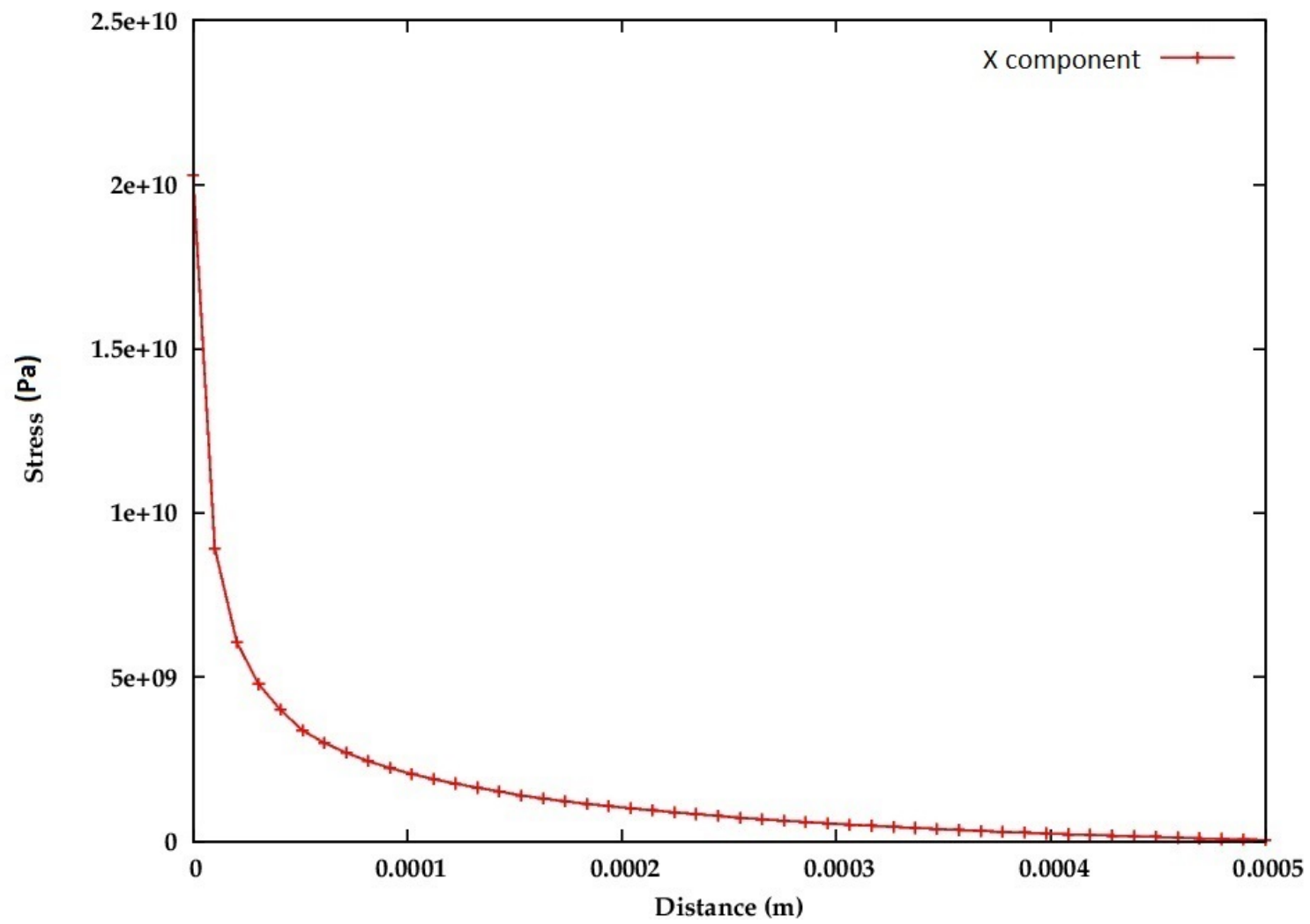

Figure A.5: Plot of the stress vs distance from the crack tip down in the plane of the crack. 


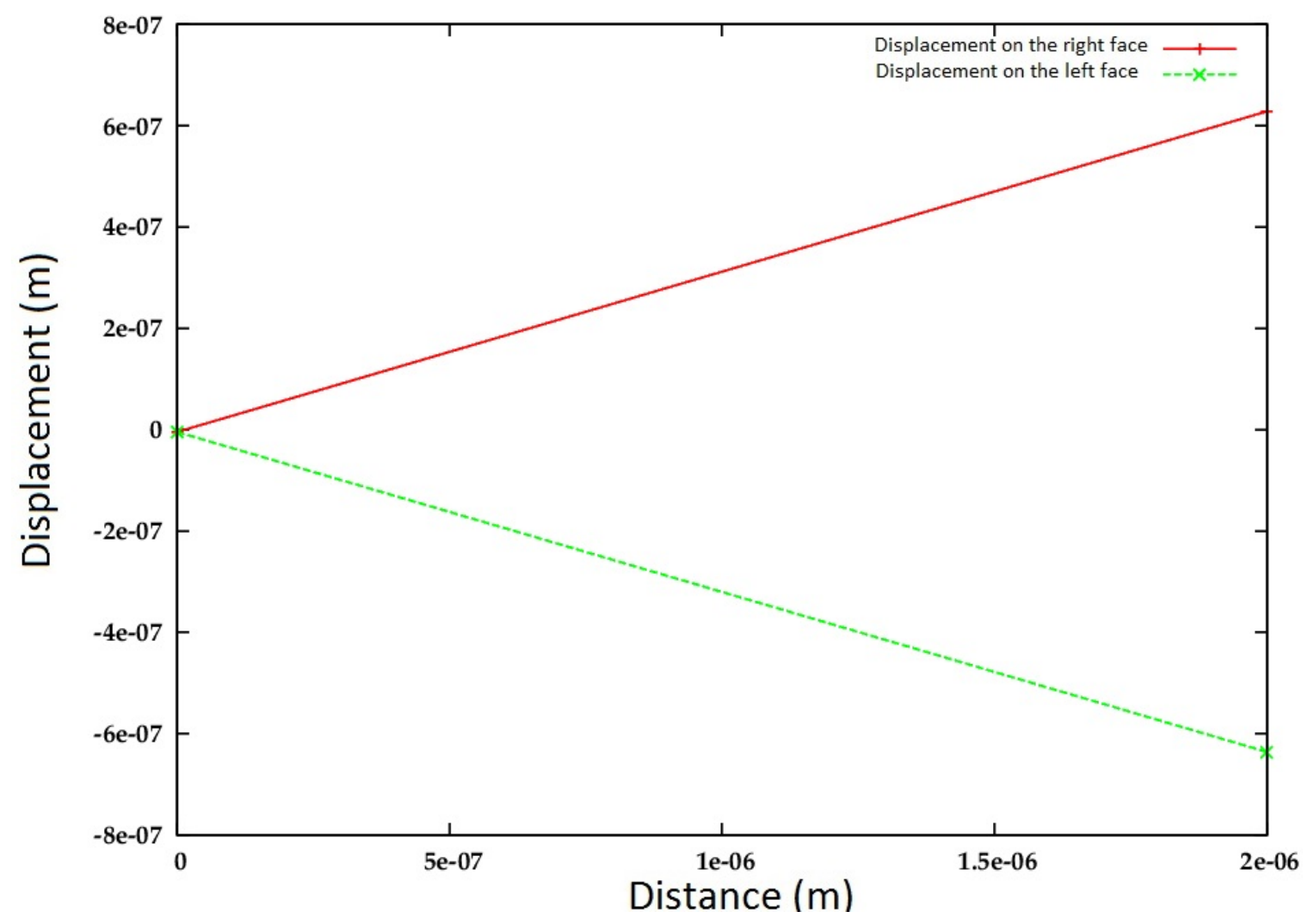

Figure A.6: Plot of the displacement vs distance from the crack tip to the distance of one element in the crack opening direction. 


\section{Appendix B}

\section{Auto-report of the fatigue analysis of the pipe girth weld for the crack length of 4}

\section{$\mathbf{m m}$}

Fatigue analysis for the current project is tailored with the help of an automated crack wizard. Results obtained in the analysis are covered in this report.

The stress intensity factor can be computed using the equation below:

$$
K_{I}=\frac{2 S}{k+1} \sqrt{\frac{\pi}{2 l}}\left[4\left(u_{b 2}-u_{b 1}\right)-\left(u_{c 2}-u_{c 1}\right)\right]=66.9 M P a \sqrt{m}
$$

where: $K_{I}$ is the stress intensity factor for mode I, $S=8.08 \cdot 10^{10}$ is the shear modulus, $l$ is the length of the element in the crack tip region, $u_{b 2}$ and $u_{b 1}$ are top displacement and bottom displacement values of the crack faces taken at the middle of an element, $u_{c 2}$ and $u_{c 1}$ are top displacement and bottom displacement values of the crack faces taken at the corner of an element, $k$ is defined as:

$$
k=(3-4 \nu) \text { (Plane srain) }
$$




$$
k=\frac{3-\nu}{1+\nu}(\text { Plane stress })
$$

where: $\nu=0.3$ is the Poison's ratio.

The result of the SIF increment $\Delta \mathrm{K}$ for the crack length of $4 \mathrm{~mm}$ can be used in Paris-Erdogan equation. 

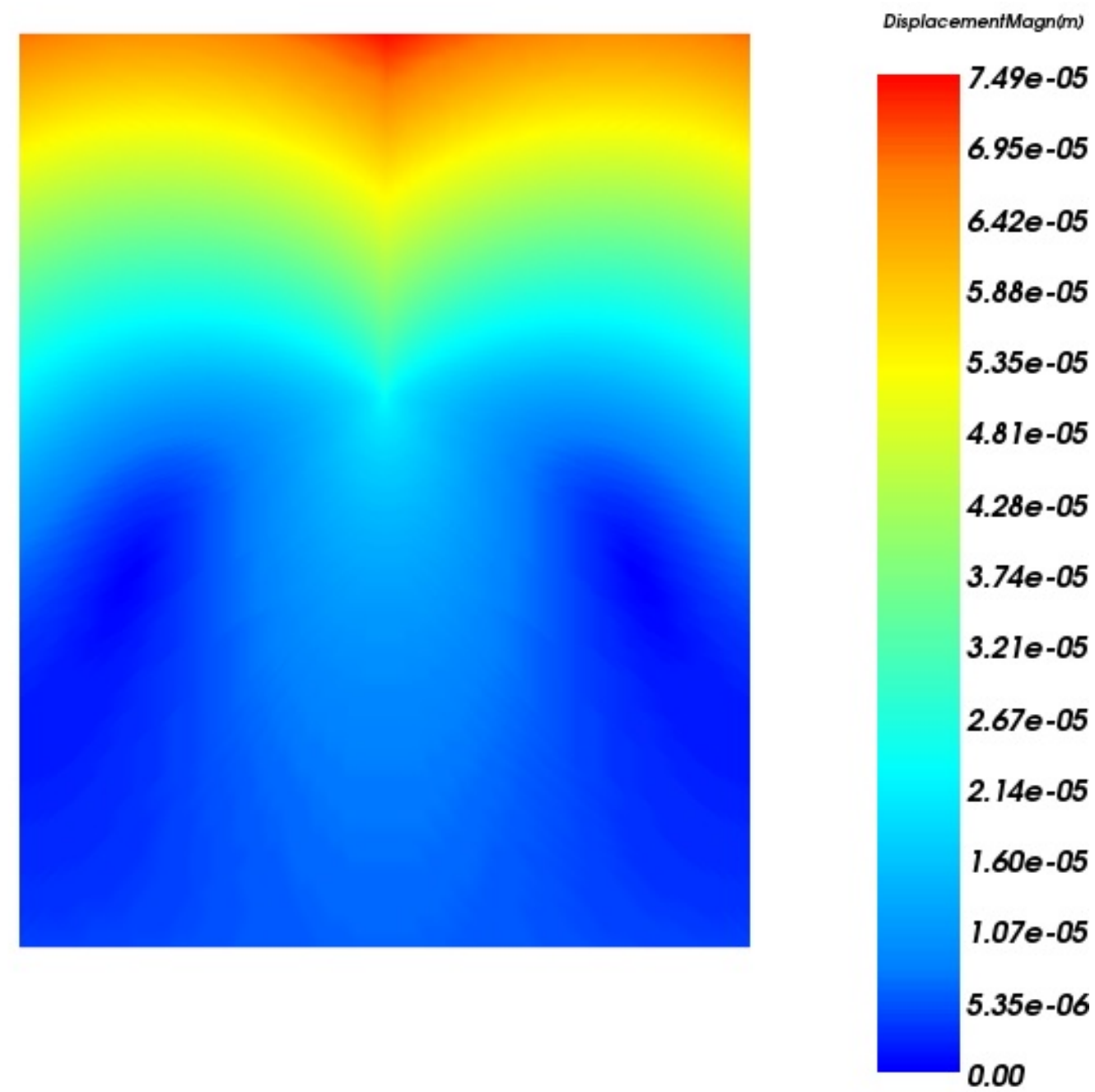

Figure B.1: The displacement field in a 2D plane strain mesh with a crack is shown. 

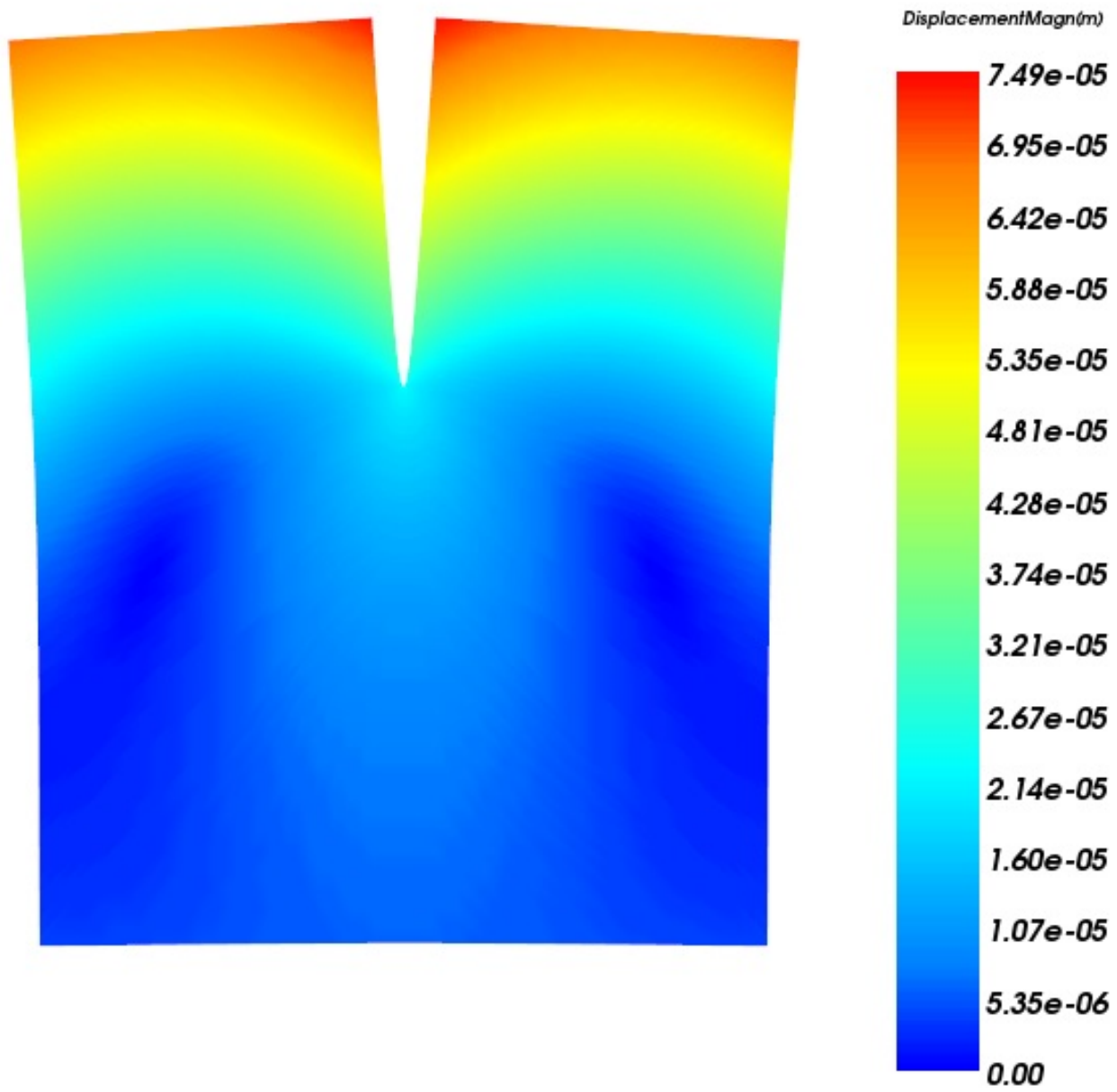

Figure B.2: The displacement field in a 2D plane strain mesh with a crack is shown. Magnified 5 times. 


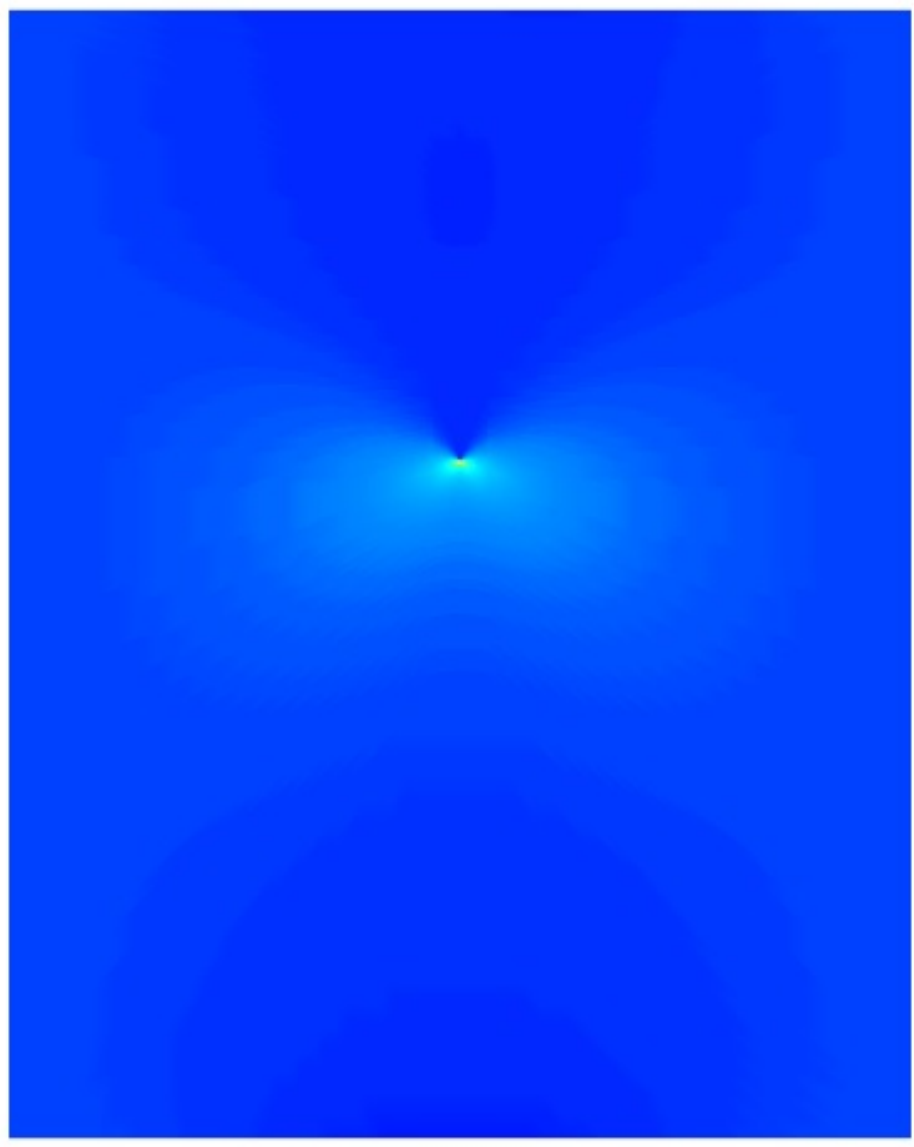

StressNodal(Pa)

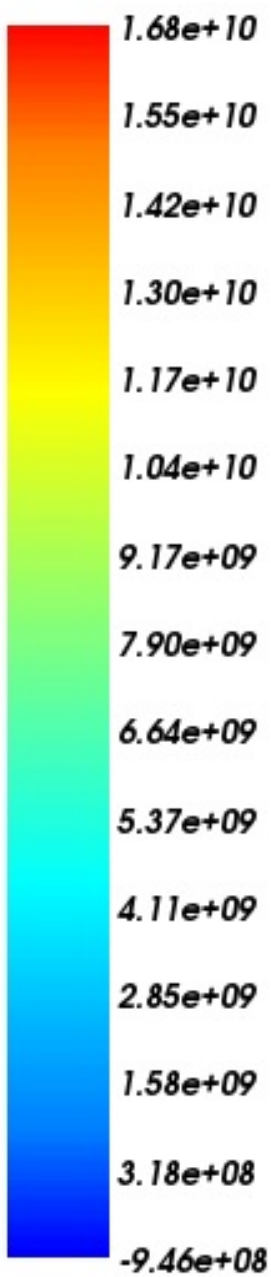

Figure B.3: The xx stress is shown in a $2 \mathrm{D}$ plane strain mesh with a crack. 


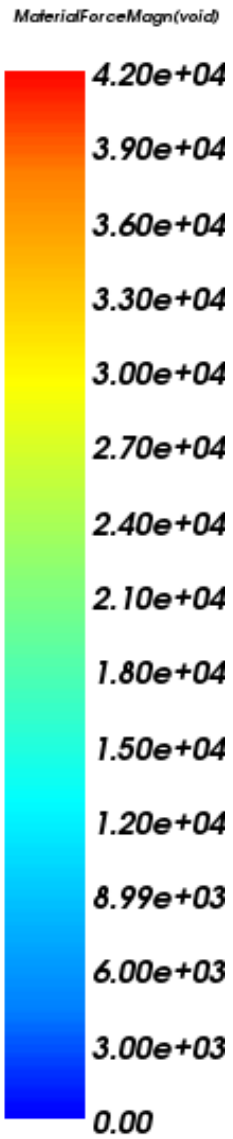

Figure B.4: The computed material force vectors near the crack tip in a 2D plane strain mesh. 


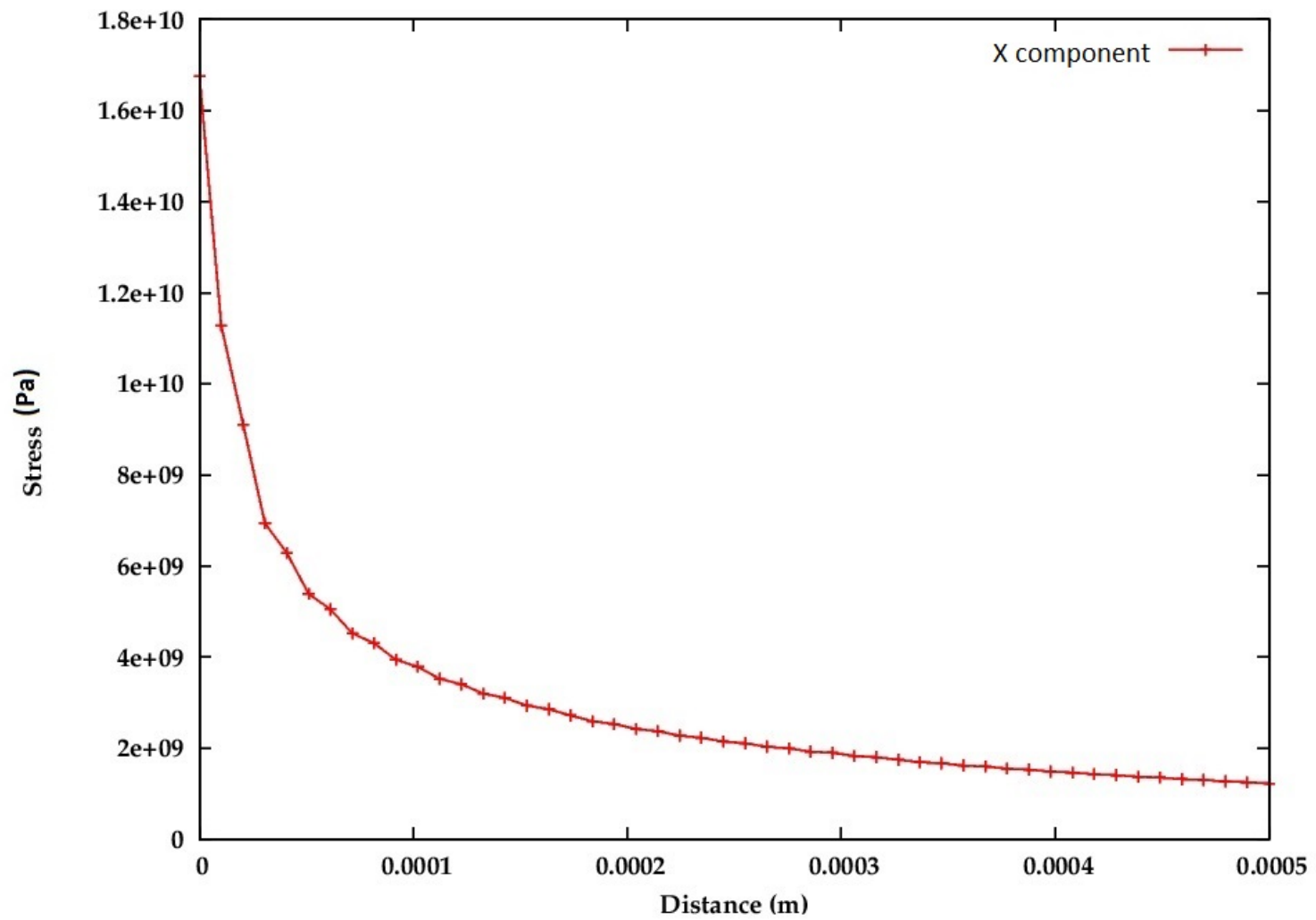

Figure B.5: Plot of the stress vs distance from the crack tip down in the plane of the crack. 


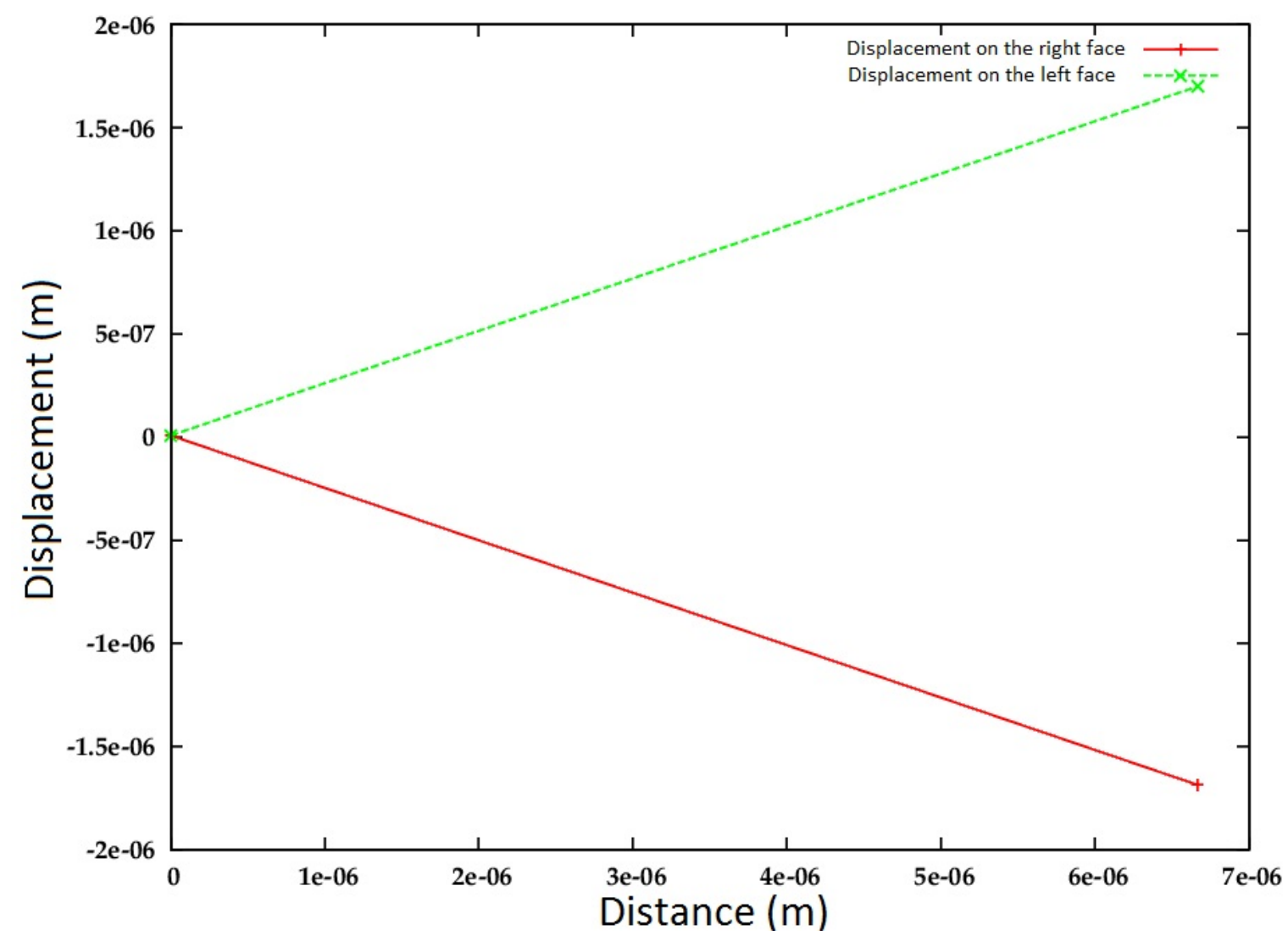

Figure B.6: Plot of the displacement vs distance from the crack tip to the distance of one element in the crack opening direction. 


\section{Appendix $\mathrm{C}$}

\section{Auto-report of the fatigue analysis of the pipe girth weld for the crack length of 6}

\section{$\mathbf{m m}$}

Fatigue analysis for the current project is tailored with the help of an automated crack wizard. Results obtained in the analysis are covered in this report.

The stress intensity factor can be computed using the equation below:

$$
K_{I}=\frac{2 S}{k+1} \sqrt{\frac{\pi}{2 l}}\left[4\left(u_{b 2}-u_{b 1}\right)-\left(u_{c 2}-u_{c 1}\right)\right]=135.9 M P a \sqrt{m}
$$

where: $K_{I}$ is the stress intensity factor for mode I, $S=8.08 \cdot 10^{10}$ is the shear modulus, $l$ is the length of the element in the crack tip region, $u_{b 2}$ and $u_{b 1}$ are top displacement and bottom displacement values of the crack faces taken at the middle of an element, $u_{c 2}$ and $u_{c 1}$ are top displacement and bottom displacement values of the crack faces taken at the corner of an element, $k$ is defined as:

$$
k=(3-4 \nu)(\text { Plane srain })
$$




$$
k=\frac{3-\nu}{1+\nu}(\text { Plane stress })
$$

where: $\nu=0.3$ is the Poison's ratio.

The result of the SIF increment $\Delta \mathrm{K}$ for the crack length of $6 \mathrm{~mm}$ can be used in Paris-Erdogan equation. 

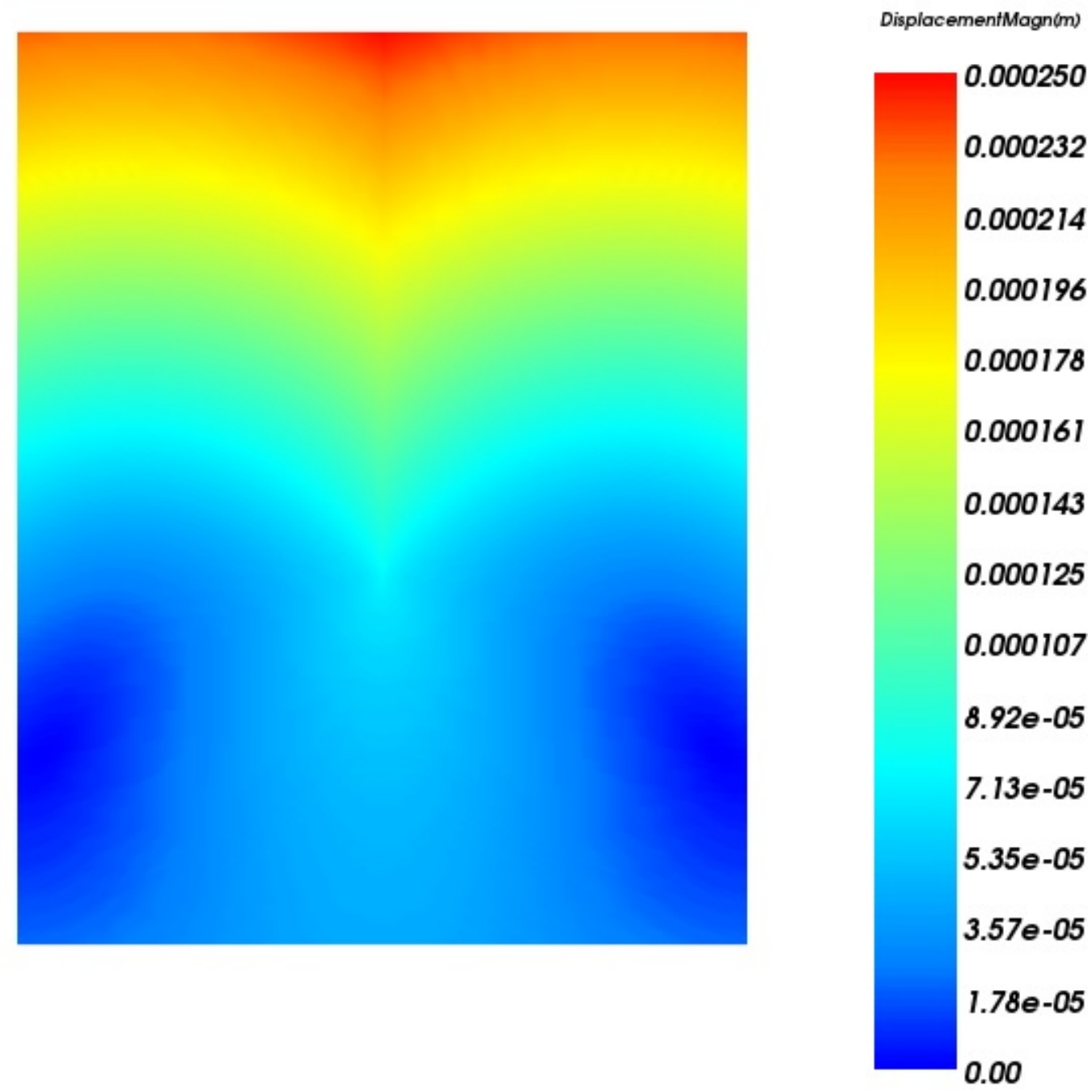

Figure C.1: The displacement field in a 2D plane strain mesh with a crack is shown. 

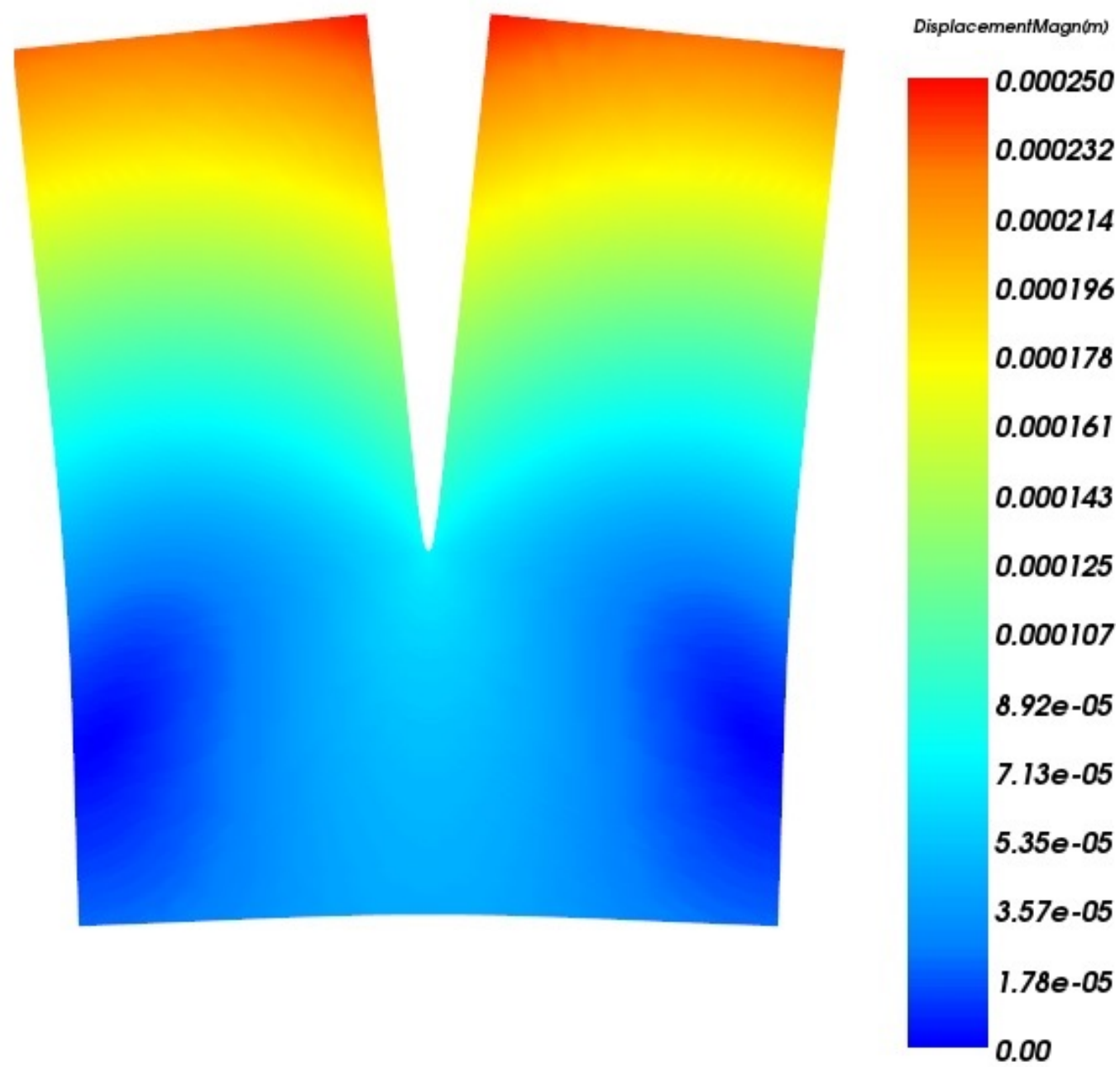

Figure C.2: The displacement field in a 2D plane strain mesh with a crack is shown. Magnified 5 times. 
StressNodal(Pa)
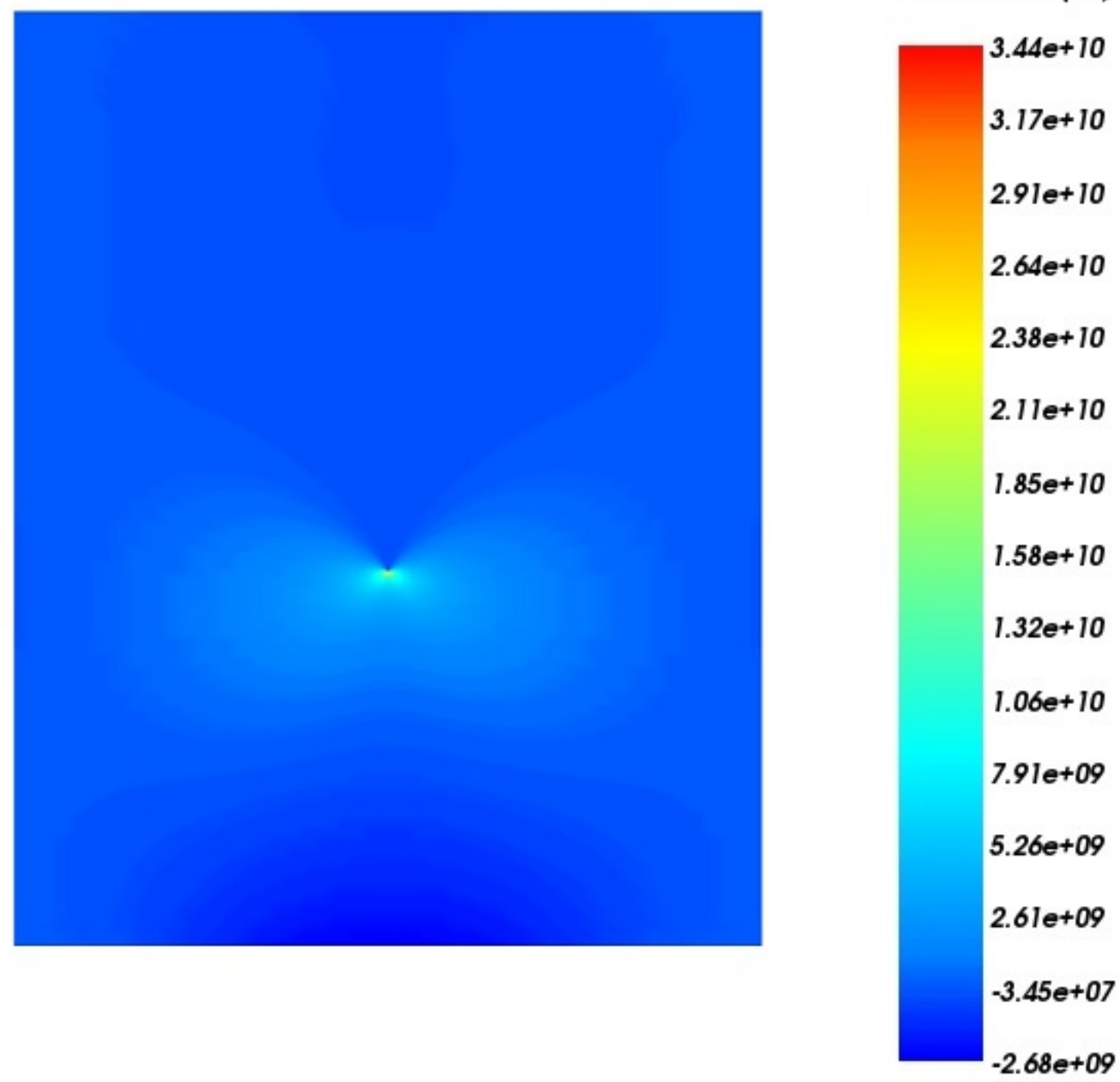

Figure C.3: The xx stress is shown in a 2D plane strain mesh with a crack. 


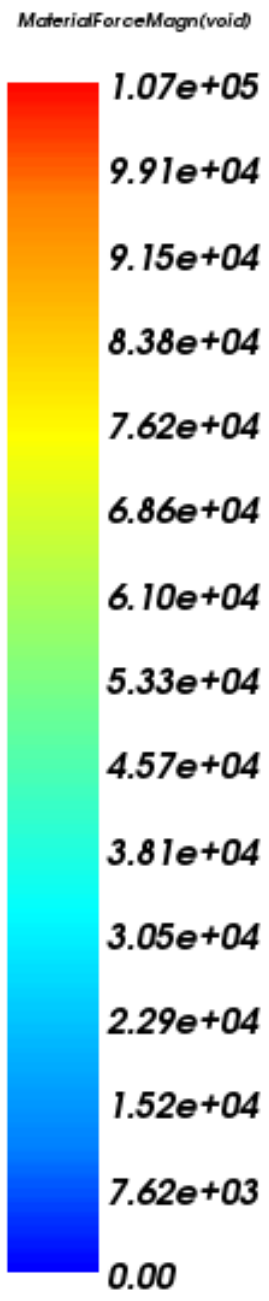

Figure C.4: The computed material force vectors near the crack tip in a 2D plane strain mesh. 


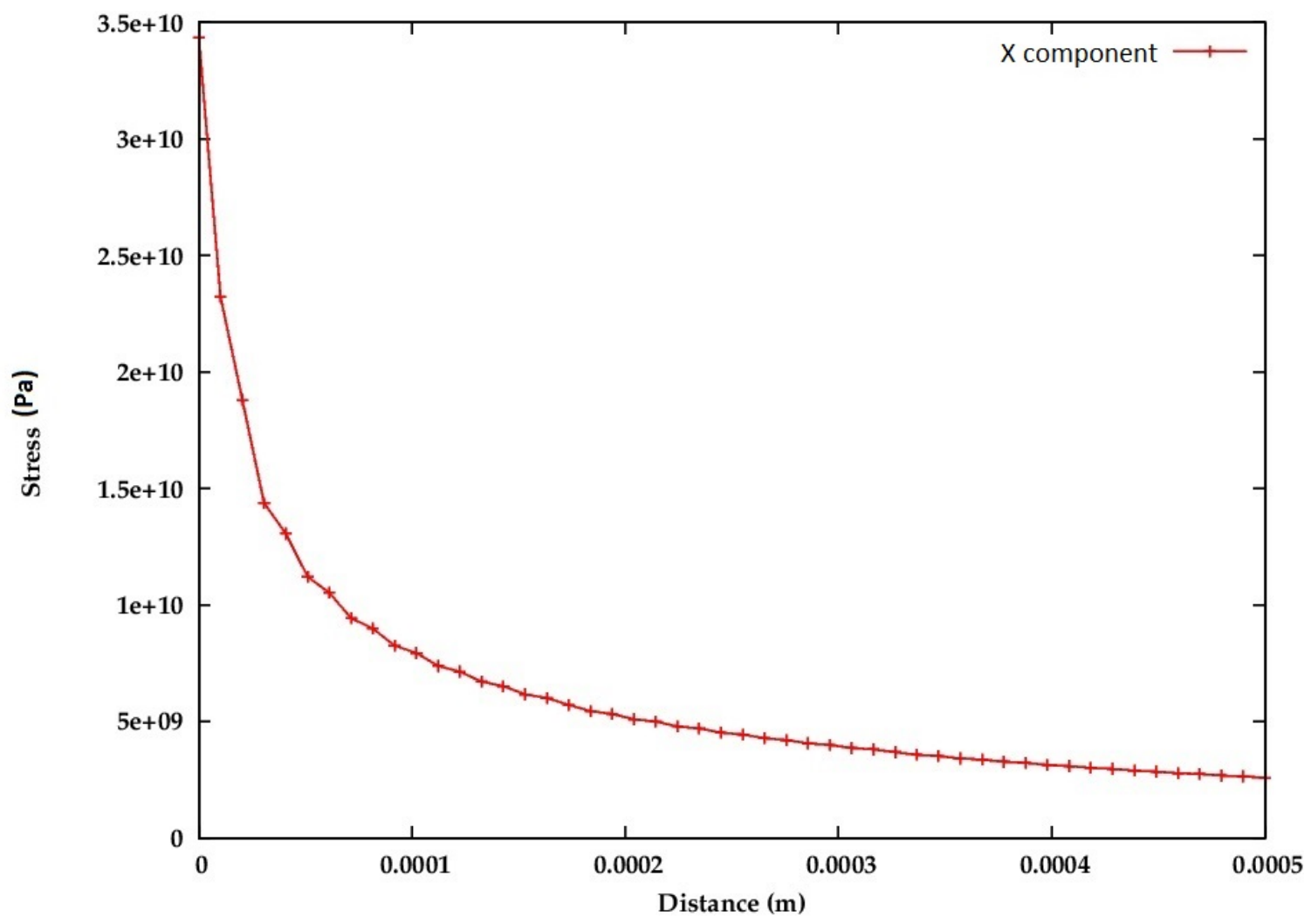

Figure C.5: Plot of the stress vs distance from the crack tip down in the plane of the crack. 


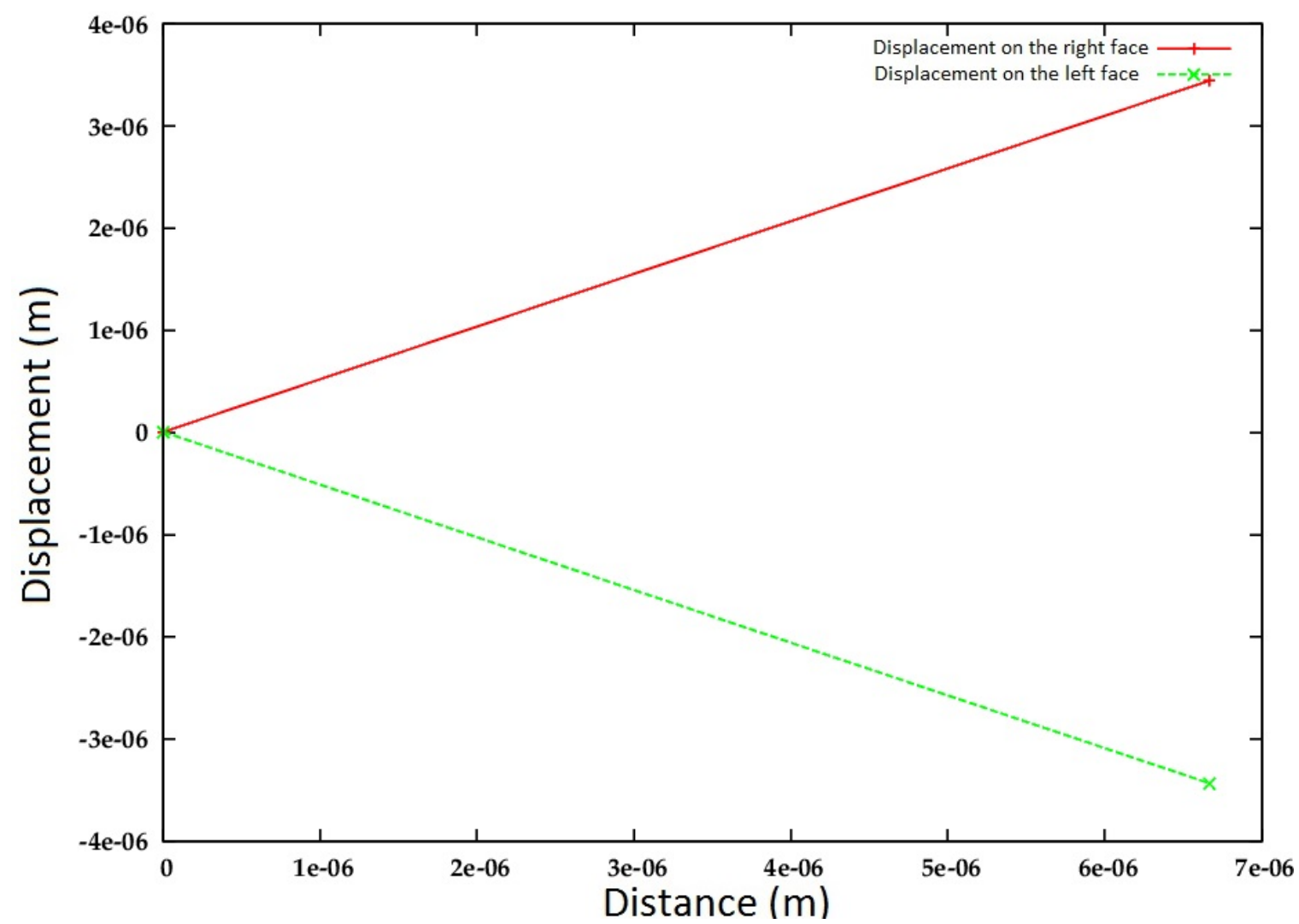

Figure C.6: Plot of the displacement vs distance from the crack tip to the distance of one element in the crack opening direction. 